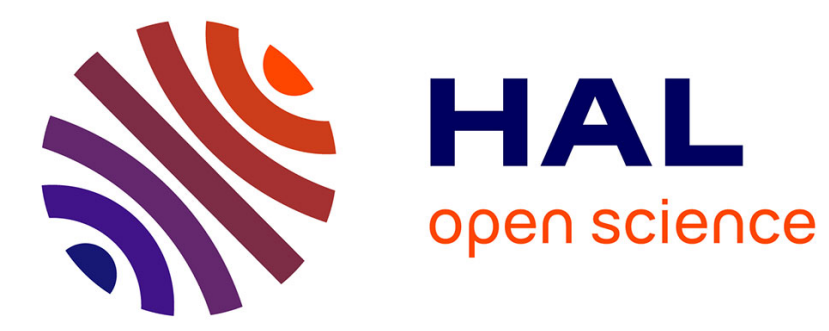

\title{
Low- and high-frequency nature of oblique filamentation modes. II. Vlasov-Maxwell simulations of collisionless heating process
}

A Ghizzo, D del Sarto

\section{- To cite this version:}

A Ghizzo, D del Sarto. Low- and high-frequency nature of oblique filamentation modes. II. VlasovMaxwell simulations of collisionless heating process. Physics of Plasmas, 2020, 27, 10.1063/5.0003698 . hal-03320849

\section{HAL Id: hal-03320849 \\ https://hal.univ-lorraine.fr/hal-03320849}

Submitted on 16 Aug 2021

HAL is a multi-disciplinary open access archive for the deposit and dissemination of scientific research documents, whether they are published or not. The documents may come from teaching and research institutions in France or abroad, or from public or private research centers.
L'archive ouverte pluridisciplinaire HAL, est destinée au dépôt et à la diffusion de documents scientifiques de niveau recherche, publiés ou non, émanant des établissements d'enseignement et de recherche français ou étrangers, des laboratoires publics ou privés. 


\section{Low- and high-frequency nature of oblique filamentation modes. II. Vlasov-Maxwell simulations of collisionless heating process}

Cite as: Phys. Plasmas 27, 072104 (2020); https://doi.org/10.1063/5.0003698

Submitted: 04 February 2020 . Accepted: 25 June 2020 . Published Online: 22 July 2020

A. Chizzo (D), and D. Del Sarto (D)

COLLECTIONS

F This paper was selected as Featured
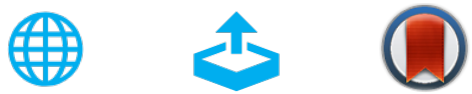

\section{ARTICLES YOU MAY BE INTERESTED IN}

Low- and high-frequency nature of oblique filamentation modes. I. Linear theory

Physics of Plasmas 27, 072103 (2020); https://doi.org/10.1063/5.0003697

Review of pulsed power-driven high energy density physics research on Z at Sandia

Physics of Plasmas 27, 070501 (2020); https://doi.org/10.1063/5.0007476

Electron kinetics in low-temperature plasmas

Physics of Plasmas 26, 060601 (2019); https://doi.org/10.1063/1.5093199

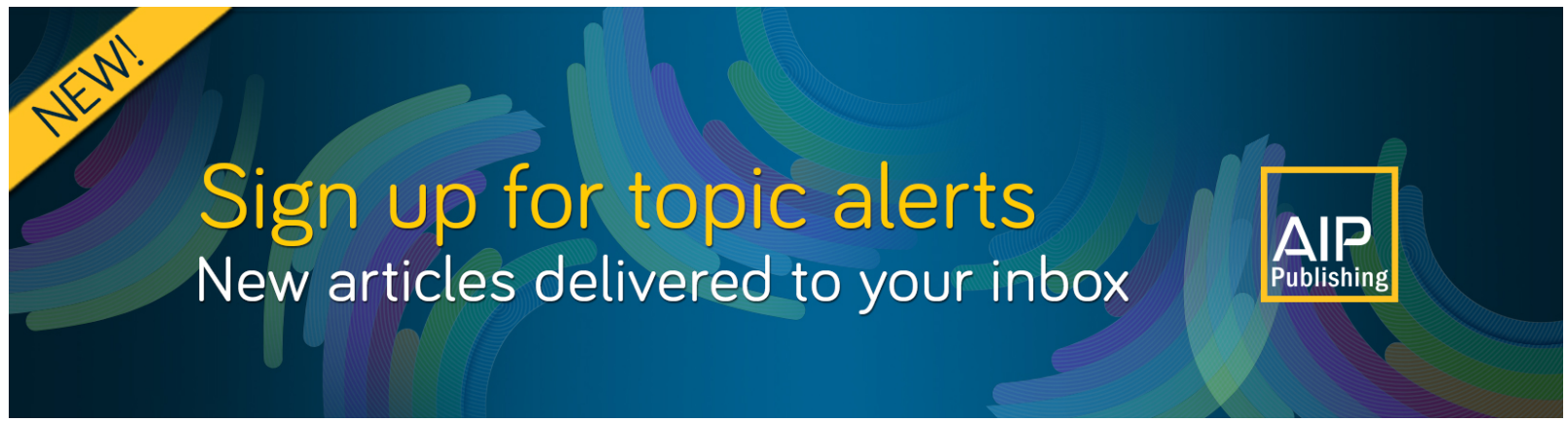




\title{
Low- and high-frequency nature of oblique filamentation modes. II. Vlasov-Maxwell simulations of collisionless heating process
}

Cite as: Phys. Plasmas 27, 072104 (2020); doi: 10.1063/5.0003698

Submitted: 4 February 2020 - Accepted: 25 June 2020 .

Published Online: 22 July 2020

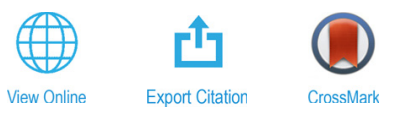

A. Ghizzo ${ }^{\text {a) }}$ (D) and D. Del Sarto (D)

\author{
AFFILIATIONS \\ Institut Jean Lamour, UMR 7198, Université de Lorraine, Campus ARTEM, BP 50840, 54011 Nancy Cedex, France
}

a) Author to whom correspondence should be addressed: alain.ghizzo@univ-lorraine.fr

\begin{abstract}
The nonlinear regime of electromagnetic oblique instabilities is investigated by means of a "noiseless" semi-Lagrangian Vlasov-Maxwell solver. Starting from an initial equilibrium configuration with two counterstreaming electron beams, qualitatively different nonlinear regimes are shown to exist depending on the nature of the solutions of the linear dispersion relation, whose properties have been discussed in the companion paper I [Ghizzo et al., Phys. Plasmas 27, 072103 (2020)]. This behavior is in contrast with existing theories of the oblique instability, which are based on the excitation of a single eigenmode at a time: nonlinear transitions toward regimes dominated by low-frequency modes are generally shown to be possible. The emphasis here is on gaining a better understanding of the multiplicity of electromagnetic oblique unstable modes and on modeling their back-reaction on plasma wave-particle interactions and energy conversion mechanisms. The latter are shown to depend on the saturation scenario of the different regimes of the oblique instability. A new regime is discussed, in which a stochastic heating occurs at the expenses of the magnetic energy first amplified by the oblique modes and in which a (reversible) violation of entropy conservation is made possible by large amplitude phase-space fluctuations of the distribution function.
\end{abstract}

Published under license by AIP Publishing. https://doi.org/10.1063/5.0003698

\section{INTRODUCTION}

Energetic particle beams with anisotropic velocity distributions in collisionless plasmas have attracted attention not only for applications to astrophysical environments, such as gamma-ray bursts or counterstreaming plasmas in cosmic outflows and shock wave sites, but also for applications to laboratory plasmas, such as those met in laser-matter interaction or fusion (tokamak) devices. A fundamental question is common to all these problems: what are the physical mechanisms that lead to plasma heating in the collisionless regime?

This question is especially relevant to turbulent environments such as the solar corona or the solar wind. In this framework, it has been recently addressed in a series of works that have considered collisionless heating mechanisms occurring in relation to pressure anisotropy, as due to intermittence processes in Alfvenic turbulence (e.g., Refs. 2-5) or as due to the anisotropic transfer from the ordered kinetic energy of the plasma flow to the components of the pressure tensor (see Refs. 6-8). The latter kind of energy transfer occurs in the presence of sheared flows, like those found in turbulence vortices, because of the action of the fluid strain on the second order fluid moment of the distribution function. ${ }^{9,10}$
As noted in the latter references, however, further channels of energy exchange between the (anisotropic) thermal and electromagnetic components can occur in a collisionless turbulent plasma because of anisotropy-driven instabilities such as Weibel-type modes and pressure-driven (kinetic) instabilities. It should be recalled indeed that, when nonideal effects that allow magnetic reconnection become nonnegligible at the small spatial scales developed by turbulence, a global heating of the plasma can occur because of the tearing-type modes that can be developed on the small current sheets convected by the fluid motion, according to the so-called "turbulent reconnection" scenario. ${ }^{11}$ The literature on this subject is huge and still increasing, but it is worth mentioning in this regard some recent studies that have evidenced how the onset of reconnection processes during the turbulent cascade can influence the turbulent spectrum and therefore the entire heating process (see Refs. 12 and 13).

Relatively less attention has been paid, so far, to the collisionless heating process that can be related to the nonlinear dynamics of Weibel-type modes. This is the subject that we partially address in the present article. Weibel-type instabilities, indeed, are related in first instance to magnetic field amplification driven by the temperature 
anisotropy, and pressure-driven instabilities are generally believed to fix threshold conditions on the ratio between the kinetic and magnetic energy densities in turbulent environments such as the solar wind. ${ }^{14-17}$

More precisely, electromagnetic Weibel-type instabilities lead to the amplification of magnetic energy, the source of the free energy coming from plasma anisotropies. The Weibel instability ${ }^{18}$ (WI) is a purely growing electromagnetic mode that can grow in an unmagnetized plasma. The source of free energy for the instability is provided by a temperature anisotropy of the electron distribution function between the perpendicular and longitudinal directions with respect to the wave vector. WI is thus an important mechanism of magnetic field generation in astrophysical phenomena, ${ }^{19,20}$ in relativistic electron beams, ${ }^{21,22}$ or in electron-positron plasmas. ${ }^{23}$ A variant of this instability, which is of major importance for laser-produced plasmas, is the current filamentation instability (CFI). In the CFI, the free energy is provided by the momentum anisotropy instead of the standard temperature anisotropy. The physical mechanisms underlying the development of both CFI and WI are very similar (see the first reinterpretation of the Weibel instability provided by Fried in terms of momentum anisotropy of two opposite beams ${ }^{24}$ ) and can be recognized in the redistribution of currents inside the plasma. Such an aspect underlies the similarities between WI and CFI and recently allowed the development of a theoretical model (the so-called multistream model ${ }^{25,26}$ ) based on a Hamiltonian reduction technique, which unifies both types of instabilities (see also Refs. 27 and 28 and more recently Refs. 29 and 30). In a counterstreaming beam-plasma configuration, the highest growth rates are typically found neither for wave vectors $\boldsymbol{k}$ that are aligned with the beam velocity [the two-stream instability (TSI)] nor for wave vectors that are normal to the beam (CFI) but rather for intermediate orientations of $\boldsymbol{k}$ : these are the oblique modes first considered in Refs. 31 and 32, where they had been named "Weibel two-stream modes" and which have been later more specifically characterized in the works by Bret et $a .^{33}$ and Gremillet et al. ${ }^{34}$ Hereafter, we refer to these modes as to the "oblique instability" (OI).

A surprising aspect arises in the long-time dynamics of the WI, which may trigger secondary plasma heating or filamentation-to-oblique transitions that are observed in particle-in-cell (PIC) simulations. $^{35-37}$ The heating mechanisms induced by CFI or WI are still poorly understood, particularly with regard to the involvement of oblique modes. As the kinetic equations are invariant under time-reversal, the transfer from kinetic to magnetic energy is, in principle, reversible. In the Vlasov equation, the reversibility in time is made possible by the fact that the filamentation process of the distribution function in the velocity space can progress indefinitely toward arbitrary small scales. One of the most significant examples is the phenomenon of echoes.

In Ref. 1, further referred to as paper I, we have performed a linear study of OI, where we have identified the roots of the dispersion relation by means of an extended fluid model in which the full pressure tensor evolution is retained. This approach leads to a polynomial dispersion relation for oblique modes, easier to be solved than the kinetic one. This has allowed us to identify different kinds of solutions: each beam exhibits low- and high-frequency roots, whose existence has been later verified by comparison with the numerical solution of the full Vlasov-Maxwell dispersion relation. Moreover, the fluid model makes it possible to identify and understand from a macroscopic point of view some of the kinetic features of these modes, whose physical interpretation appears difficult when a full kinetic Vlasov approach is used.

Guided by the linear analysis performed in paper $\mathrm{I}^{1}$ in this second article, we investigate these classes of solutions by studying the nonlinear saturation of oblique instabilities with semi-Lagrangian (SL) Vlasov-Maxwell simulations. Here, the emphasis is on the evolution of oblique and filamentation modes in the nonrelativistic or (weakly) relativistic regime. The onset of oblique instabilities leads to a pattern of current filaments of variable sizes, depending on the nature of the roots of the linear dispersion relation. The emergence of these filaments is quite general, and simulations show that such solutions can be excited in both nonrelativistic and relativistic collisionless regimes. Furthermore, SL Vlasov-Maxwell simulations illustrate the efficiency of the oblique instability in converting back magnetic energy into kinetic energy: we will show that the interplay between the two classes of oblique modes ("low" and "high" frequency) discussed in paper $\mathrm{I}^{1}$ can lead to an efficient secondary conversion from electromagnetic to kinetic (thermal) energy of the plasma.

This paper is organized as follows: In Sec. II, we recall the Vlasov-Maxwell (VM) model and its main conservation constraints, and we discuss several thermodynamical aspects of the model. Section III gives a summary of the physical problem we investigate. Section IV presents the numerical semi-Lagrangian scheme and the initial conditions used. In Sec. V, we present and discuss the results of the study of low-wavenumber oblique modes. Section VI concerns the discussion of the new stochastic heating scenario observed in the presence of high wavenumber oblique modes. Finally, conclusions are in Sec. VII.

\section{THE VLASOV-MAXWELL MODEL \\ A. Model equations}

We assume ions to constitute a neutralizing background. This is motivated by the fast time scales of the phenomena we are interested in. We then consider only the evolution of the electron distribution function $f=f(\boldsymbol{x}, \boldsymbol{p}, t)$, which obeys the relativistic Vlasov equation,

$$
\frac{\partial f}{\partial t}+\frac{\boldsymbol{\rho}}{m \gamma} \cdot \nabla_{x} f+e\left(\boldsymbol{E}+\frac{\boldsymbol{p} \times \boldsymbol{B}}{m \gamma}\right) \cdot \nabla_{p} f=\left(\frac{\partial f}{\partial t}\right)_{C G},
$$

where $\gamma=\sqrt{1+\frac{p^{2}}{m^{2} c^{2}}}$ is the Lorentz factor and $\nabla_{x}$ and $\nabla_{p}$ are the gradients with respect to the space and to the momentum coordinates, respectively. Here, $m$ is the electron rest mass, $c$ the light velocity, and $e<0$ the elementary electron charge. The Vlasov equation is then coupled with the Maxwell equations in a self-consistent way. The electromagnetic field $(\boldsymbol{E}, \boldsymbol{B})$ obeys

$$
\begin{gathered}
\frac{\partial \boldsymbol{E}}{\partial t}-c^{2} \nabla_{\boldsymbol{x}} \times \boldsymbol{B}+\frac{\boldsymbol{J}}{\varepsilon_{0}}=0, \\
\frac{\partial \boldsymbol{B}}{\partial t}+\nabla_{\boldsymbol{x}} \times \boldsymbol{E}=0, \\
\nabla_{\boldsymbol{x}} \cdot \boldsymbol{E}=\frac{\rho}{\varepsilon_{0}} \\
\boldsymbol{\nabla}_{\boldsymbol{x}} \cdot \boldsymbol{B}=0 .
\end{gathered}
$$

Note that in Eq. (1), we have included a rhs term, normally not present in the writing of the continuum Vlasov equation, in order to evidence the phenomenon of coarse graining (CG) that would appear after the introduction of an elementary cell of size $h$ into the phase space. This 
feature is an unavoidable ingredient of the numerical integration of the Vlasov equation on an Eulerian domain, which is necessary to keep into account while interpreting the numerical results: the averaging scheme for the distribution function over an elementary cell is indeed a crucial element of any back-reaction study.

In statistical physics, coarse graining is the procedure of smoothing the fine, small-scale structure of the physical system. Clearly, there are many ways to perform it, and the chosen technique must be adapted to the physical or mathematical properties of the system under consideration. In the Vlasov approach, from a mathematical point of view, the asymptotic convergence of $f$ holds only in the weak sense, and the velocity derivatives, in modulus, grow quickly at large times. This leads to the phenomenon of filamentation of $f$ in the velocity space and to an energy (and information) transfer from low to high wave numbers, which resembles a weak turbulence-type cascade. The Vlasov-Maxwell system is reversible as long as this transfer of information to smaller scales can proceed indefinitely. Thus, as it happens in the free transport equation as $\partial f / \partial t+\boldsymbol{v} \cdot \nabla_{x} f=0$, oscillations of $f$ should occur in a very "ordered way." This mathematical regularity was called by Villani "gliding regularity." ${ }^{27,58}$ In the Fourier space of velocities, such a regularity appears in the form of an information packet that moves along $\lambda$, where $\lambda$ is the Fourier variable of $v$. The semi-Lagrangian scheme used here (see Sec. IV) preserves such a property since the velocity differentiation corresponds to multiplication by $2 i \pi \lambda$ after Fourier transform: a regularity control may be thus realized in Fourier space, when a numerical grid is introduced in the simulation. An alternative approach to control the smoothing of filaments in phase space has been proposed by Klimas et al. ${ }^{61}$ In any case, when the size of the box in velocity is finite, information is lost as the packets cross the boundaries. Information is, however, conserved in a continuum velocity space since the extension of the Fouriertransformed domain, $\lambda_{\max }=\pi / \Delta v$, goes to infinity as the size of the cell in the velocity domain, $\Delta v$, tends to zero. Note that the entropy defined using Shannon's formula,

$$
S=-k_{B} \int \frac{d^{3} x}{V} \int f \ln f d^{3} p,
$$

is perfectly preserved when $h$ tends to zero since it is a Casimir invariant of the Hamiltonian Vlasov-Maxwell system. However, if an average is performed on the elementary cell with a finite volume $h$, the information inside this cell is lost and the entropy increases. This is the coarse graining mechanism introduced by Ehrenfest. In numerical modeling, this effect is played by the introduction of a numerical mesh that, as fine as it can be, introduces a discretization of the phase-space domain.

In the following, we are going to show that, in addition to this numerically induced smoothing, a physical mechanism can also lead to this type of effect, especially during a transition from an oblique mode with a large wave vector to an oblique mode with a small wave vector. This mechanism can be similar to an inverse cascade process and can lead to a smoothing phenomenon in the configuration space since the micro-filamentation can be smoothed during the transition between the two types of modes. We will see that this mechanism has some impact on the entropy conservation, too, which is not of numerical nature but can be understood in more physical terms if the notion of entropy is "corrected": indeed, although (6) provides the quantitative definition of entropy usually adopted in kinetic plasma simulations, and which we will also refer to, we will see in Sec. II B that the definition of $S$ can be refined to express the notion of entropy for a beam-plasma configuration in a more appropriate, thermodynamical sense [cf. Eq. (19)].

In Eqs. (2) and (4), the source terms as the electron current density $J$ and the charge density $\rho$ are defined by the following relations:

$$
\begin{gathered}
\boldsymbol{J}=e \int \frac{\boldsymbol{p}}{m \gamma} f d^{3} p, \\
\rho=e \int f d^{3} p-e n_{0} .
\end{gathered}
$$

In (8), $n_{0}$ is the fixed background ion density; $\boldsymbol{J}$ and $\rho$ satisfy the continuity equation,

$$
\frac{\partial \rho}{\partial t}+\nabla_{x} \cdot \boldsymbol{J}=0
$$

which expresses the local charge conservation. Condition (9) is satisfied automatically provided that the Poisson equation (4) is initially verified.

\section{B. Basic properties of the model and thermodynamical aspects}

We recall here the fundamental properties of the Vlasov model and in particular its links with some thermodynamics aspects. To simplify the presentation and to make connection with the standard, continuum Vlasov model, we assume here that the size of the elementary cell $h$ be zero (i.e., that $\left.(\partial f / \partial t)_{C G}=0\right)$. On multiplying the Vlasov equation (1) by $p_{i}$ and integrating each component " $i$ " over all momenta, we have

$$
\frac{\partial \boldsymbol{C}}{\partial t}+\nabla_{x} \cdot \tilde{\Pi}=e n \boldsymbol{E}+\boldsymbol{J} \times \boldsymbol{B}
$$

where $C=\int p f d^{3} p$ is the average momentum density, $\tilde{\Pi}=\int p v f d^{3} p$ is the material stress tensor density of plasma, and the (electron) current density is given by

$$
\boldsymbol{J}=e \boldsymbol{n} \boldsymbol{u}=e \int \boldsymbol{v} f d^{3} p .
$$

It is worth drawing attention to the distinction between the material stress tensor $\tilde{\Pi}$ in Eq. (10) and the pressure tensor $\Pi=n m\langle(\boldsymbol{v}-\boldsymbol{u})$ $(\boldsymbol{v}-\boldsymbol{u})\rangle$ that we have introduced in paper I: ${ }^{1}$ while the two quantities are, of course, related, the latter tensor, being defined with respect to the rest frame of the flow, expresses only the "thermal" contribution to stress deformations in the plasma. The average momentum density $\boldsymbol{C}$ also intervenes in the flux term of the (local) energy density conservation law, which we can express in the form

$$
\frac{\partial}{\partial t}\left[K+\frac{1}{2} \varepsilon_{0}\left(E^{2}+c^{2} B^{2}\right)\right]+\nabla_{x} \cdot\left(\boldsymbol{C}+\frac{\boldsymbol{E} \times \boldsymbol{B}}{\mu_{0}}\right)=0 .
$$

In Eq. (12), $K=m c^{2} \int \gamma f d^{3} p$ is the relativistic kinetic energy density. Equation (12) evidences the possibility to convert "kinetic" (or thermal) energy into "electromagnetic" energy or vice versa. From a thermodynamical point of view, it is well known (see Ref. 41, p. 6) that the relativistic Maxwell-Jüttner distribution function can be derived by maximizing the free energy of the system $F=\epsilon-T S$, while the 
number $\langle N\rangle$ of particles is kept constant. The extension to a relativistic beam was realized by Davidson and Yoon in Ref. 42. It was shown by these authors that for a beam-plasma system, the thermodynamical equilibrium may be characterized by the Helmholtz free energy defined as

$$
F=\epsilon-v_{b} \tilde{C}_{y}-T_{b} \tilde{S}-T_{b} N=\text { Const. }
$$

Here, $v_{b}$ and $T_{b}$ are the velocity (in modulus) and temperature of the beam, $\epsilon$ is the total (particle-plus-field) energy, $\langle N\rangle \simeq N$ is the average number density, $\tilde{S}$ is a redefinition of Shannon's entropy, which is compatible with this thermodynamical analysis and which we will discuss later [cf. Eq. (19)], and $\tilde{C}_{y}$ is the projection along the axis of the beams, which we suppose to be $y$, of the vector,

$$
\tilde{\boldsymbol{C}}=\int \frac{d^{3} x}{V}\left[\boldsymbol{C}+\frac{\boldsymbol{E} \times \boldsymbol{B}}{\mu_{0}}\right] .
$$

This is the integral over the volume $V$ of the momentum density $C$ to which electromagnetic field contributions have been added. We can therefore name $\tilde{\boldsymbol{C}}$ the "total momentum vector" and $\tilde{C}_{y}$ its axial component. For quantitative (e.g., numerical) applications, we may use $V=L_{x} L_{y} L_{z}$ for a $3 D$ system or $V=L_{x} L_{y}$ for a $2 D$ spatial system. It is also assumed that the spatial integrations in Eq. (14) and hereafter are over a periodic spatial domain. In this case, $\tilde{C}_{y}$ is a constant of motion because of periodic boundary conditions in the $y$ space coordinate. The authors make then use of the global conservation constraints corresponding to the conservation of $\epsilon$, of $N$, of the entropy $S$ and of the total axial momentum $\tilde{C}_{y}$ to obtain the formal expression of the measure of the field perturbation [Eq. (11) in Ref. 42],

$$
\begin{aligned}
\mathcal{F}(t)-\mathcal{F}(0)= & \int \frac{d^{3} x}{V} \int d^{3} p\left\{-\left(m c^{2} \gamma-v_{b} p_{y}\right)\left(f_{b}-f_{b 0}\right)\right. \\
& -T_{b}\left[f_{b} \ln \left(\frac{f_{b}}{f_{b \max }}\right)-f_{b 0} \ln \left(\frac{f_{b 0}}{f_{b \max }}\right)-\left(f_{b}-f_{b 0}\right)\right] .
\end{aligned}
$$

In Eq. (15), $f_{b}(\boldsymbol{x}, \boldsymbol{p}, t)$ represents the distribution function of the beam population, which evolves according to the continuum Vlasov equation [i.e., according to Eq. (1) without the $(\partial f / \partial t)_{C G}$ term] from its initial condition given by $f_{b 0}=f_{b}(\boldsymbol{x}, \boldsymbol{p}, t=0)$. It is then straightforward to show from (15) that $\mathcal{F}$ takes a maximum value whenever $f_{b}$ describes a relativistic thermal equilibrium distribution with temperature $T_{b}$ and average axial velocity $v_{b} \boldsymbol{e}_{y}$, which reads

$$
f_{b}=f_{b \max } \exp \left[-\frac{\left(m c^{2} \gamma-v_{b} p_{y}\right)}{k_{B} T_{b}}\right] .
$$

With the proceeding notations, these constraint conditions read

$$
\begin{gathered}
\epsilon=\int \frac{d^{3} x}{V}\left[\int m c^{2} \gamma f_{b} d^{3} p+\frac{1}{2} \varepsilon_{0}\left(E^{2}+c^{2} B^{2}\right)\right]=\text { const } \\
N=\int \frac{d^{3} x}{V} \int f_{b} d^{3} p=\text { const } \\
\tilde{S}=-k_{B} \int \frac{d^{3} x}{V} \int f_{b} \ln \left(\frac{f_{b}}{f_{b \max }}\right) d^{3} p=\mathrm{const}
\end{gathered}
$$

$$
\begin{aligned}
\tilde{C}_{y} & =\int \frac{d^{3} x}{V}\left[C_{y}+\left(\frac{\boldsymbol{E} \times \boldsymbol{B}}{\mu_{0}}\right)_{y}\right] \\
& =\int \frac{d^{3} x}{V}\left[\int p_{y} f d^{3} p+\left(\frac{\boldsymbol{E} \times \boldsymbol{B}}{\mu_{0}}\right)_{y}\right]=\text { const. }
\end{aligned}
$$

We further define $\alpha_{b}=m c^{2} /\left(\gamma_{b} k_{B} T_{b}\right)$, which is a dimensionless parameter that measures the thermal spread. The normalization constant is then given by $f_{b \max }=n_{b 0} \alpha_{b} /\left[4 \pi m^{3} c^{3} \gamma_{b} K_{2}\left(\alpha_{b}\right)\right]$, where $\gamma_{b}=\left(1-v_{b}^{2} / c^{2}\right)^{-\frac{9}{2}}$ is the Lorentz factor and $K_{n}$ is the modified Bessel function of the second kind of order $n$.

The expression of the entropy $\tilde{S}$ in Eq. (19), already obtained by Davidson and Yoon, shows a possible connection with information theory and differs from the standard definition (6) of the entropy often used in plasma physics because of the explicit dependence on $f_{\text {bmax }}$.

From the point of view of information theory, indeed, entropy is not a property of the system but rather a measure of "our ignorance" about the system. The equilibrium state that can be observed in the asymptotic state after the phenomenon of "coarse-graining" corresponds in this sense to a maximum of the entropy because information about the initial conditions has been lost except for conserved quantities. However, in the presence of large fluctuations, whose relative amplitude in Eq. (19) is "compared" to the value of $f_{\text {bmax }}$ through the logarithm contribution, the amount of information at the microscopic level may change and the entropy can so display large variations due to second-order effects. It is worth highlighting that this feature does not contradict the conservation of entropy (6) defined as a Casimir invariant of the Vlasov equation and discussed in Sec. II A: the condition of gliding regularity discussed by Mouhot and Villani ${ }^{57}$ and Villani ${ }^{58}$ implies indeed the absence of strong fluctuations of $f$ in the phase-space, which is required in the known demonstrations that grant the convergence of the phase-space Casimir integrals of the Vlasov-Maxwell system (see also Refs. 59 and 60). Instead, this fact suggests that also Shannon entropy conservation can be violated in the presence of large amplitude fluctuations. Notably, we are going to show that both an increase and a decrease in Shannon's entropy are made possible by the presence of large amplitude fluctuations of the distribution function, which are related to filamentation and oblique modes.

\section{THE PHYSICAL PROBLEM}

As stated at the beginning of Sec. II A, we are interested in electron-induced instabilities only, which justify the neglecting of ion dynamics as a first approximation.

We consider an initial configuration with two symmetric counterstreaming electron beams, which hereafter we assume to stream along the $y$ direction (see Fig. 1). From now on, we will label the parameters related to the two streams with the indices $i=1$ and 2 . However, in order to make connection with a more general case in which the two beams are not symmetric, we will sometimes refer to this configuration as to a "beam-plasma." The temperatures are chosen to be isotropic in each beam rest frame. This rules out the pure Weibel type mode, driven indeed by the pressure anisotropy in the plane of the beams and perturbation, and its coupling with electrostatic and oblique modes. As discussed in paper $\mathrm{I},{ }^{1}$ there are at least three instability classes in this configuration: the current filamentation instability (CFI), the electrostatic two-stream instability (TSI), and the 
<smiles>CC(C)C</smiles>

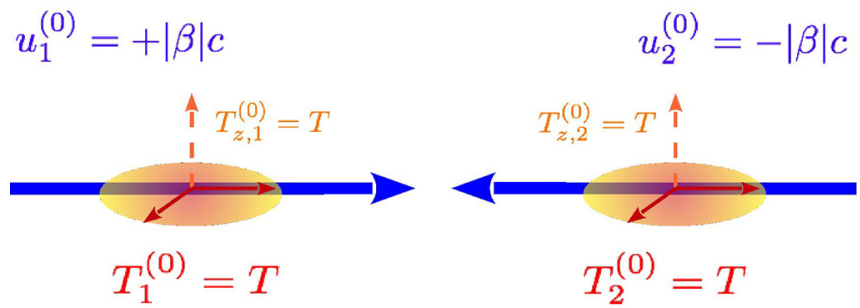

FIG. 1. Sketch of the equilibrium configuration (counterstreaming symmetric electron beams) perturbed by an oblique wave-vector. An in-plane isotropic temperature is assumed so to exclude initial coupling with pure Weibel-modes.

oblique instability (OI). It is well known that TSI modes are exactly longitudinal $\left(k_{x}=k_{z}=0\right)$, while CFI modes are perpendicular $\left(k_{y}=k_{z}=0\right)$ with respect to the direction of the beams. If perturbations both parallel and perpendicular to the stream flows become unstable, the filamentation-type instability is referred to as oblique. As done in paper $\mathrm{I},{ }^{1}$ we will hereafter consider only the problem of harmonic perturbations whose space coordinate dependence is twodimensional, that is, $\boldsymbol{k}=\left(k_{x}, k_{y}, 0\right)$.

As previously mentioned, and as it is known from a series of previous studies (see Refs. 31-37), the CFI should dominate during the first stage of a cold symmetric beam evolution $\left(n_{1}=n_{2}\right)$. Filamentation modes, however, are nontime resonant (i.e., they have $\operatorname{Re}(\omega)=0$ ), whereas oblique modes, although having smaller growth rates in comparison to those of the CFI modes, have a finite phase velocity and thus can non-negligibly contribute to the long-time behavior of the plasma through wave-particle resonances. The knowledge of the dynamics of low-frequency (LF) oblique modes is therefore essential for assessing the possible mechanism of the wave-particle interaction in the nonlinear regime of these beam-plasma instabilities. This, however, is difficult to be realized by following the usual approach to the linear study of kinetic instabilities, which consists in searching (numerically) the most unstable growing mode among the roots of the kinetic dispersion relation. This task is made particularly difficult by the nonpolynomial form of the latter, which is further complicated in the relativistic regime.

In paper $\mathrm{I},{ }^{1}$ we have discussed how this problem has been overcome by studying the dispersion relation of the OI with an extended fluid model that includes the full pressure tensor dynamics. On the one hand, the amount of information contained in the second order fluid moments of the distribution function suffices to describe the physics of these linear modes (as it has been a posteriori verified by comparison with the solutions of the full Vlasov-Maxwell linear system), and on the other hand, this approach considerably simplifies the algebra by leading to a polynomial dispersion relation. This facilitates the analysis and interpretation of the roots of the latter in regions of the parameter space where oblique modes can be excited. In particular, our analysis has revealed the existence of a new resonant branch of low frequency modes (noted B in Fig. 2 and which will be discussed in Sec. V B), in addition to what we can consider to be the "standard" high frequency solution, already known in the literature (this has been noted as case A in Fig. 2 and will be discussed in detail in Sec. V A). The linear analysis performed in paper $\mathrm{I}^{1}$ has also revealed the existence of regions in the parameter space where low- and high frequency solutions exist close to each other.

A cartography of these two classes of modes in the normalized $\left(k_{x}, k_{y}\right)$-plane (whose axes are displayed on a log-log scale) is shown in Fig. 2, where the contour-lines represent equal values of the growth rates of two types of OI solutions of the full-kinetic dispersion relation: in case A (lower lobe), only the high-frequency roots have been selected, whereas in case B (upper lobe), the low frequency roots have been chosen. The roots corresponding to the two other types of instabilities, CFI and TSI, have not been represented here. The fact that the two distinct neighboring roots, $\mathrm{A}$ and $\mathrm{B}$, overlap in a region of the $\left(k_{x}, k_{y}\right)$ space makes their identification and distinction difficult. Both modes can be resonant in time since each mode has a real frequency (see paper $\mathrm{I}^{1}$ ). It is then clear that both modes can be resonantly excited for the same values of $k_{x}$ and $k_{y}$ if the appropriate resonant conditions on the respective phase-velocities are met.

Let us now turn the attention to the nonlinear regime. It is well established from the previous literature that two different mechanisms exist, which lead to the saturation of Weibel-type instabilities. At small wavenumbers (i.e., $k d_{e}<1, d_{e}=c \omega_{p}^{-1}$ being the electron skin depth), saturation is produced by the so-called Alfvèn mechanism, ${ }^{43,44}$ which imposes an upper limit to the current density carried by the particles. At large wavenumbers (i.e., $k d_{e} \gtrsim 1$ ), instead, the saturation is widely recognized to occur due to magnetic trapping (MT). ${ }^{26,30,44-46}$

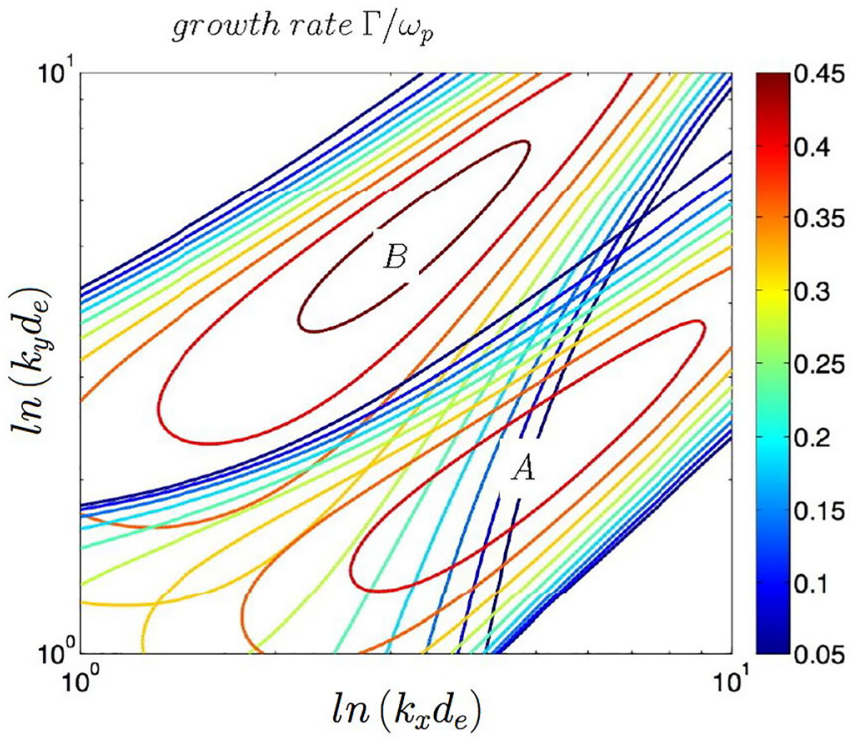

FIG. 2. Contour-plot of the growth rate of the oblique filamentation instability $\eta / \omega_{p}$ in the $\left(k_{x} d_{e}, k_{y} d_{e}\right)$ plane, on a log-log scale. The two classes of solutions ( $A$ and $B$ ) obtained from the full kinetic dispersion relation are displayed. Case $B$ (upper lobe) corresponds to the linear low-frequency solution, while case A (lower lobe) is related to the linear high-frequency solution. 
However, the present study extends this analysis to take into account the contribution of the "electrostatic" particle trapping in the $k d_{e} \gtrsim 1$ nonlinear regime of the OI. This occurs at saturation of the low-frequency regime of the instability (case B in Fig. 2) when $k d_{e} \gg 1$ (let us say when $k d_{e} \gtrsim 10$ ) because of the resonant transition of the oblique mode from small to large wave numbers (i.e., from $k d_{e} \sim 1$ to $k d_{e} \sim 30$ ). This leads to a stochastic heating mechanism in which the transfer from kinetic to magnetic energy, typical of anisotropy-driven electromagnetic instabilities, can be reversed (this mechanism will be discussed in detail in Sec. VI).

It must be noted that the current filaments associated with the CFI and oblique modes correspond to fluctuations of the distribution function in the phase-space, which, depending on their amplitude, can affect the entropy conservation/evolution in the Vlasov-Maxwell system (cf. Sec. II B).

Although the beam plasma evolution that we are going to discuss can have implications for kinetic turbulence processes, especially in collisionless environments, e.g., the solar wind, the problem we are going to focus on differs from fully developed (2D) turbulence. In the present study, only some specific classes of oblique modes are linearly excited at each time, and they are accurately selected among the unstable roots that linear analysis has evidenced to exist in a region of the parameter space where the small-to-large wave number resonant transition of modes can, in principle, occur. The latter and its implications for the fundamental properties of the Vlasov-Maxwell system (i.e., phase-space filamentation, energy conversion mechanisms, and entropy fluctuations) are the focus of the present study, which is made possible by the almost noiseless character of the semi-Lagrangian solver that we are going to discuss in Sec. IV. The emergence and the growth of oblique modes with large values of $k d_{e}$ can indeed lead to a stochastic behavior when two oblique modes are excited.

The motivation of our study is then twofold: first, numerical semi-Lagrangian Vlasov simulations have been carried out to verify the possible transition of the oblique instability from small wavenumbers (referred to as the case B in Fig. 1) to large wavenumbers; second, the possible magnetic-to-kinetic energy conversion associated with this transition is investigated. Its characterization in terms of turbulence is, however, beyond the purpose of the present article, and it will be discussed in some future work.

\section{NUMERICAL MODEL}

There are three main classes of numerical methods that have been used so far to solve the VM system: the Lagrangian approach, the Eulerian approach, and the semi-Lagrangian approach. The numerical studies performed in this work rely on a semi-Lagrangian solver.

\section{A. Lagrangian, Eulerian, and semi-Lagrangian schemes: Qualitative comparison of main drawbacks and advantages}

These three classes of numerical schemes differ because of the strategy with which the Vlasov equation is represented and integrated in the phase-space and are often regarded as complementary based on their performance for the description of specific problems of plasma physics.

The Lagrangian approach, typical of the particle-in-cell (PIC) method, consists in sampling initial positions of particles in phase space (the marker load phase), in following their trajectories in phase space (this is accomplished by the "pusher" or particle mover part of the algorithm), and then in obtaining the source terms for the field equations at every time step (step of charge and current assignment). This procedure is indeed equivalent to compute the characteristics of the Vlasov equation.

The Eulerian approach consists in discretizing the phase space on a fixed grid and in applying finite differences, finite volume schemes, and/or 2D or 3D Fourier transform (the spectral method) for the differential operators. The evolution of the distribution function is then followed by direct integration of the Vlasov equation over time.

The semi-Lagrangian (SL) approach uses a fixed phase space grid, like in Eulerian schemes, but obtains the values of $f$ at the next time step by tracing back in time the trajectories ending at each grid point and by interpolating the distribution $f$ at the foot of the orbits. Thus, in the SL approach, a new population of "markers" is chosen at each time step, initially located on grid points in phase space, and finally, the characteristics of the Vlasov equation are solved in the backward direction.

Both Eulerian or SL methods are sometimes referred to as "Vlasov" codes to mark the difference with respect to PIC-codes in the way the distribution function is represented: in the former, a continuous function is sampled on a discrete mesh, whereas in the latter, a finite number of particles are made evolve; they approximate the distribution function in the continuum limit.

Each of the three methods has its own advantages and drawbacks, although all share, of course, the mean field approach, which is, at the basis, of the Vlasov description: Coulomb interactions between charged particles are replaced by a mean field calculated from Maxwell's equations, where the microscopic fluctuations (physically related to the non-continuum nature of the plasma gas) are averaged over the Debye length, $\lambda_{D}$.

PIC codes probably represent the most popular methods, especially apt to perform 3D simulations for comparison with experiment results. In these codes, the stage of charge and current density assignment can be viewed as a Monte Carlo integration having a statistical error proportional to $1 / \sqrt{N_{\text {part }}}$, where $N_{\text {part }}$ is the number of markers. When the focus is on the description of collective properties of the Vlasov plasma, PIC codes exhibit, however, a paradoxical situation, as the introduction of a spatial grid (for instance of size $\Delta x \sim \lambda_{D}$ ) allows one to describe the collective effects through the mean field approximation but at the price of reintroducing individual effects due to the finite number of superparticles or markers. These individual effects are often considered as a "numerical noise" or, more properly, a numerical amplification of the statistical noise. Its measure can be quantified in terms of a $g_{\text {PIC }}$ parameter, as shown by Feix and Bertrand ${ }^{64}$ (see also Ref. 65). This "PIC-graininess factor" depends only on numerical parameters according to $g_{P I C}=1 /\left(n_{q p}(\Delta x)^{d_{x}}\right)$. These parameters are the quasi-particle density $n_{q p}=N_{\text {part }} / V$, related to the number of markers $N_{\text {part }}$ and to the volume $V$ of the plasma, the space mesh size $\Delta x$, and the space dimension of the domain $d_{x}$. Since the real graininess factor $g$ associated with Coulomb binary collisions needs to go zero in the mean field approach at the basis of the Vlasov model (although it must be pointed out that in a real low-collision plasmas, $g$ is small but not zero), keeping the coherence of the superparticle model with the Vlasov equation needs $g_{P I C}$ to be as the smallest as possible. This corresponds to large values of the number of markers, 
which, in the end, makes the computational cost of PIC models equivalent to that of nonparticle Vlasov codes.

On the other hand, Eulerian and semi-Lagrangian schemes are said to be noiseless since the distribution function is represented on an Eulerian grid in the phase-space. As a consequence, these schemes are not subject to the issue of statistical noise due to the sampling of markers, which is critical in the Lagrangian-PIC method. A drawback is present, however, as the use of finite difference schemes for computing differential operators in space introduces numerical dissipation, whose effect on the numerical integration of the otherwise Hamiltonian Vlasov-Maxwell system has to be assessed by grid convergence studies. At the same time, it must be noted that this numerical dissipation is also what makes steady-state simulations possible, by permitting the convergence of the otherwise collisionless system to "attractors" in the phase-space.

In the end, both PIC and Vlasov codes exhibit some nonHamiltonian features that are not present in the original continuum Vlasov model but whose nature (and effects) differ in the two types of numerical approaches (PIC vs nonparticle). While, in Eulerian schemes, numerical dissipation is produced by discretization of the operators, this is not the only effect in Lagrangian schemes for which the filamentation of the distribution function in the phase space gives rise to a numerical increase in the statistical "noise" (in place of pure dissipation only). This is an element that goes beyond the "equivalence" between semi-Lagrangian and PIC finite element methods, which has been discussed by Bermejo. ${ }^{47}$ Moreover, while filamentation at a sub-grid scale usually leads to a numerical instability in Vlasov codes or at least to a loss of invariants' conservations, thus leaving scarce doubts about the physical interpretation of the numerical results, the stability of PIC codes with respect to this effect typically occurs at the expenses of the numerical result being affected by an "artifact" particle noise, whose impact on the numerical solution and on its physical interpretation is a priori difficult to assess.

Further differences can then be recognized among Vlasov codes, between Eulerian and semi-Lagrangian approaches, as far as time integration is concerned.

First, although both Eulerian and semi-Lagrangian schemes for the Vlasov-Maxwell system must fulfill Courant-Friedrichs-Lewy (CFL) stability-type conditions, it must be noted that in the semiLagrangian approach, these are due only to the integration of the Maxwell equations: the backward integration along the characteristic of the Vlasov equation frees the latter from CFL-type constraints, which are present instead in the direct time integration performed with Eulerian Vlasov codes. In particular, in the electrostatic Vlasov-Poisson limit, the semi-Lagrangian approach allows the use of time steps much larger than in usual explicit time integrations of Eulerian schemes, thanks to the lack of CFL constraints.

Second, further important differences become manifest when relativistic effects are included in nonparticle codes. The technique of the time splitting operator may, in principle, be applied in Eulerian schemes to avoid a multi-dimensional interpolation. However, special relativity, through the introduction of the Lorentz factor, introduces a strong topological constraint: as a consequence, a 3D interpolation in momentum space must be used, whereas one-dimensional interpolations remain possible in the coordinate space. Thus, the main difficulty lies in finding an appropriate interpolation scheme, where a compromise between dissipation and accuracy has to be met. In this regard, a further indicator of the accuracy of the scheme for the integration over long time intervals is given by Casimir's or energy conservation. In order to address this point, different time integration schemes for nonparticle codes have been compared since the 1990s, although this comparison has been usually restricted to the one-dimensional Vlasov-Poisson system (for the nonlinear Landau problem, for instance)-see, e.g., Ref. 74 and more recently Ref. 75. In Ref. 74, in particular, three different interpolation schemes were considered: the cubic spline interpolation method, ${ }^{38,40,48,76}$ the Fourier-Fourier method $^{17}$ (based on the use of a splitting method allowing the treatment of each advection term separately, a method that differs from the standard spectral approach), and the flux balance method (FBM) ${ }^{78} \mathrm{~A}$ comparison between these interpolation techniques was investigated in terms of accuracy and dissipation for the nonlinear Landau problem, and it was pointed out how the conservation/variation of the $L^{2}$ norm and the Shannon entropy $S$ allows an accurate measure of both the dissipation and accuracy of the numerical interpolation scheme. In Ref. 38, it has been then evidenced how the semi-Lagrangian approach, using multi-dimensional advections, be a viable substitute to the explicit, forward time-splitting scheme, which, in the relativistic regime, has been shown ${ }^{79}$ to be not applicable for $1 \mathrm{D}$ advections in the velocity space because of the failure of integral conservations.

In the present work, where we address Weibel-type instabilities in a relativistic regime by focusing on the implication of phase-space filamentation for collisionless collective phenomena, we have therefore opted for the use of a semi-Lagrangian approach.

\section{B. The semi-Lagrangian scheme: General features}

The numerical scheme we use to integrate the Vlasov-Maxwell system on an Eulerian grid is based on the semi-Lagrangian method, which is perfectly suited to describe the nonlinear dynamics of the kinetic electromagnetic modes we are interested in. The basis for the semi-Lagrangian (SL) scheme $e^{38,40}$ is the method first presented by Cheng and Knorr $^{48}$ who introduced two new concepts for the numerical integration of the Vlasov equation: the first one is the method of time splitting, which consists of separating the time integration of the Vlasov equation in multiple steps, each related to a "partial advection" of the distribution function; the second one is the idea of numerically integrating, at each step of the advection, the Vlasov equation along its characteristics. As the extremities of the characteristics do not coincide with the grid points, a reconstruction of the distribution function on the Eulerian grid is required by using interpolation techniques.

The semi-Lagrangian VLasov Electro-Magnetic (VLEM) solver that we have here adopted uses some improvements with respect to previous SL Vlasov-Maxwell codes, ${ }^{38,40}$ like a new algorithm of charge conservation, that have been described in Ref. 39. These allow a full relativistic treatment by using cubic $B$-spline interpolation in the momentum space and local Hermite interpolation in space. The former is required because of the intensive parallelization of the code, and the latter allows highly relativistic regimes to be processed. The Vlasov equation (1) is integrated in the phase-space for (formally) $(\partial f / \partial t)_{C G}=0$ by applying a splitting scheme (a method of fractional time advancement), which consists in treating separately the space advection due to the convective term $\boldsymbol{p} \cdot \nabla_{x} f$, related to the particle free streaming, and the advection due to the acceleration-plus-rotation term with respect to the $\boldsymbol{p}$ coordinates, $[\boldsymbol{E}+(\boldsymbol{p} \times \boldsymbol{B}) /(m \gamma)] \cdot \nabla_{\boldsymbol{p}} f$. 
Since the distribution function that solves the Vlasov equation is constant along its characteristics, by knowing $f$ at $t_{n}=n \Delta t$, we can compute the solution at time $t_{n+1}$,

$$
f\left(\boldsymbol{x}, \boldsymbol{p}, t_{n+1}\right)=f\left(\mathbf{X}\left(t_{n} ; \boldsymbol{x}, \boldsymbol{p}\right), \mathbf{P}\left(t_{n} ; \boldsymbol{x}, \boldsymbol{p}\right), t_{n}\right) .
$$

Here, $\mathbf{X}\left(t_{n} ; \boldsymbol{x}, \boldsymbol{p}\right)$ and $\mathbf{P}\left(t_{n} ; \boldsymbol{x}, \boldsymbol{p}\right)$ solve the characteristic differential equations,

$$
\frac{d \mathbf{X}}{d t}=\frac{\mathbf{P}(t)}{m \gamma(\mathbf{P}(t))}
$$

and

$$
\frac{d \mathbf{P}}{d t}=\mathbf{F}(\mathbf{X}(t), t)
$$

where we have used $\boldsymbol{F}=e \boldsymbol{E}+\mathbf{P} \times \boldsymbol{B} /(m \gamma)$ and $\gamma$ $=\sqrt{1+P^{2}(t) /\left(m^{2} c^{2}\right)}$ to indicate the Lorentz factor. Then, $f$ is approximated using the grid points of the computational domain $(\boldsymbol{x}, \boldsymbol{p})$, and after each time step, its value at the origin of the characteristics $\mathbf{X}$ and $\mathbf{P}$ is used to compute its new value. The whole distribution function required for the next time step is then calculated from the values it takes on the grid points by using the aforementioned high order interpolation methods ( $B$-spline and Hermite polynomial).

The VLEM code is a fully parallelized hybrid openMP-MPI version of the solver, which displays an extremely low level of numerical noise. The $B-$ splines, used in the SL scheme in the advection of momentum, prevent any reflection of the information at the boundaries of the box, and thus, no (numerical) information is re-injected inside the system. The loss of information, occurring after filaments reach the size of the elementary cell, depends on the numerical resolution of the phase-space mesh.

Semi-Lagrangian codes for the integration of the Vlasov equation on an Eulerian grid have been used for many years and have made it possible to describe parametric three-wave instabilities (such as the Raman-type instability involving the decay of a laser pump wave into a plasma wave and a scattered electromagnetic wave $e^{49-51}$ ) with unequaled accuracy. This has allowed, for example, the study of the action transfer between the plasma mode and trapped and accelerated particles while preserving the conservation of classical invariants (mass, energy, and entropy) with high values of precision. These types of codes are nowadays also used to describe gyrokinetic turbulence in tokamaks with very good accuracy. ${ }^{52}$ Furthermore, the SL method had been already exploited for the integration of continuity-type equations also in addition to plasma physics, for example, by Staniforth and Cote $^{53}$ for the integration of fluid equations for weather and climate simulations.

Studies about the convergence of semi-Lagrangian schemes have been, therefore, made in different contexts of applications. A first convergence study of the semi-Lagrangian scheme has been performed by Bermejo $^{54}$ for application to weather equations and by Falcone and Ferreti $^{55}$ for pure advection equations. The analysis of the scheme in terms of accuracy and efficiency was made by Bartello and Thomas. ${ }^{56}$ More recently, the convergence of the scheme applied to the twodimensional Vlasov-Maxwell system (the code VLEM) has been discussed in Ref. 39, together with numerical tests concerning current filamentation and Weibel instabilities.

\section{Numerical dissipation and "lack of noise" in the semi-Lagrangian scheme}

As anticipated in Sec. II, entropy fluctuations represent an important feature of the nonlinear processes that we are going to discuss in Secs. V and VI. Since the entropy variation in a numerical scheme is also intimately related to the dissipative and/or collisional features introduced by discretization of the continuum model, some remarks about these features in the semi-Lagrangian solver are due.

Of course, from a numerical point of view, entropy is never fully preserved in Vlasov simulations on an Eulerian grid. Indeed, the Vlasov equation is subject to the filamentation process of $f$ in the velocity space that leads to the formation of filaments of the distribution function in phase space. When a filament reaches the size of the elementary cell of the numerical phase space grid, some information is irretrievably lost, and this leads to an irreversible entropy production.

This property is related to numerical dissipation/diffusion introduced by the discretization of the differential operators, and it is evidently encountered in all codes integrating the Vlasov-Maxwell system. It must be pointed out, however, that the overall effect of this numerical dissipation (and diffusion) on the numerical integration does not uniquely depend on the truncation error of the differential operators since it also depends on the smoothing effects that the interpolation methods (e.g., B-spline and Hermite-polynomials in the VLEM case) have on each specific physical process. That is, the rate at which information is irreversibly lost because of coarse graining depends, in a complex way, on the rate at which filamentation occurs, on its spatial "(ir)regularity" in the phase-space, and on how this is dealt with the interpolation scheme, depending on its accuracy and on the Eulerian phase-space sampling. This is a subject that, at the best of our knowledge, has been poorly addressed so far and would deserve some dedicated studies on its own. Moreover, we are going to show that large amplitude fluctuations of the distribution function related to phase-space filamentation can also reversibly violate entropy conservation of the Vlasov-Maxwell system (cf. Sec. II B). This is why we have generically expressed both dissipative features of the numerical integration and collisionless/reversible violations of the Vlasov mean field equation by means of the inclusion of the "effective" right hand side term $(\partial f / \partial t)_{C G}$ in Eq. (1).

At the same time, it must be pointed out that the irreversible dissipation due to the combined effect of truncation of differential operators, phase-space filamentation, and interpolation, is, in principle, different from the irreversible loss of information that is introduced in the numerical scheme because of effective particle collisions, which, in PIC-codes, can be associated with the finite number of quasi-particles. The latter more properly corresponds to an "effective collision term" that can be associated with PIC codes and that is responsible for their intrinsic noise: we have indeed seen (Sec. IV A) that it can be expressed as an effective numerical graininess factor likened to particle collisions, $(\partial f / \partial t)_{\text {PIC }} \sim g_{\text {PIC }}$. This effective $g_{\text {PIC }} \neq 0$ factor exists since the beginning of any PIC simulation and, in the PIC-type numerical integration of the Vlasov equation, would correspond to the $a b$ initio presence of a $(\partial f / \partial t)_{P I C}$ rhs term replacing $(\partial f / \partial t)_{C G}$ in Eq. (1). On the contrary, the numerical CG induced by phase-space filamentation at scales below the resolution of the Eulerian phase-space grid is initially zero in a Vlasov simulation (such as the case of the VLEM code): the numerical CG is a feature that becomes manifest at a later stage of the numerical simulation and depends on the physical problem 
considered, which influences the rate at which filamentation develops. After a long time, indeed, the shape of $f$ in a continuum phase-space domain may be very complicated, and the distribution can tend to a stationary meta-equilibrium, although only in the mathematical sense of weak-convergence: the average value of any continuous $f$ tends to its equilibrium value, while the entropy associated with the particle distribution must remain constant. However, if we divide the phasespace into cells and if, at each time step of the numerical integration, we supplement the phase-space dynamics induced by the Vlasov equation with a recurrent average of $f$ inside each cell (which is what is practically done during the numerical interpolation of $f$ on the phasespace grid points), entropy can increase, in agreement with Ehrenfest's CG concept. Depending on whether this smoothing induces a permanent loss of information or a "redistribution" of it among different scales of the phase-space (like in the plasma echo problem), the increase in entropy may be or may not be irreversible.

It is in this sense that we say the semi-Lagrangian scheme to be noiseless (with respect to PIC codes), while, of course, it is subject to numerical dissipation and diffusion.

At the same time, since we are going to discuss entropy variations that are not of numerical nature and, notably, a reversible entropy increase unrelated to numerical dissipation, a brief discussion of dissipation in the semi-Lagrangian algorithm is due, at least for problems in which numerical dissipation is the only effect expected to affect conservations of the Vlasov-Maxwell system.

In this regard, we have already mentioned in Sec. IV A that Ref. 74 evidenced how measuring the $L^{2}$ norm or the entropy is a good test to check the convergence and efficiency of the numerical scheme. In particular, these quantities provide a good check for the method of the interpolation used in the splitting scheme and usually allow us to assess the role of dissipative effects introduced by the interpolation techniques in the semi-Lagrangian approach. By addressing the reader to Ref. 74 for details, we recall here that in the study of nonlinear Landau damping of a beam-plasma instability, a fast entropy increase was observed for the three different interpolation methods compared there (cubic spline, Fourier-Fourier, and flux balance method): the entropy reaches a maximum level at about the time the electrostatic instability saturates, and then, no tendency to further growth was observed. Although small differences could be observed during the growth of the $L^{2}$ norm and entropy $S$, all methods exhibited the same final state characterized by very close values of the entropy. This indicates that the asymptotic regime does not depend on the numerical interpolation used. Increasing the sampling of the distribution function in the phase space, the steady state was also reached but at later times. Such a final state was characterized by the same value as before of both the entropy and other Casimir invariants, most of which (e.g., energy) were very well conserved.

Concerning more specifically the differences related to dissipation among interpolation schemes in Vlasov codes, we first recall to be well known that the semi-Lagrangian method does not preserve the positivity of $f$ and that the amplitude of spurious oscillations increases when nonlinear effects occur. However, the conservative flux balance method seems to be more dissipative than the cubic-spline semiLagrangian method used in VLEM: in Refs. 74 and 75, the entropy was found to increase faster for the FBM method than for the cubicspline semi-Lagrangian one, and for the FBM scheme, the strong dissipation can be ascribed to the averaging step introduced in the flux transport scheme and to the elimination of small-scale details of the distribution function, aimed at stabilizing the scheme.

We conclude by noting that further improvements to Vlasov (i.e., nonparticle) schemes have been devised: a positive flux conservative (PFC) method has been proposed in Ref. 80, which is a high-order extension of the FBM scheme. High order interpolation schemes are less dissipative but can lead to unphysical oscillations and, in general, to negative values of $f$ (see Ref. 81). Conservative semi-Lagrangian schemes have been also developed (see Ref. 82), with the introduction of slope limiters. Furthermore, new reconstruction methods, namely, "weighted essentially nonoscillatory" (WENO) techniques have been also proposed. ${ }^{83}$ More recently, different test problems for the onedimensional treatment of the Vlasov-Poisson system, usually concerning the free particle advection, the plasma wave echoes, or the nonlinear Landau damping, have been reconsidered. Among the different numerical schemes presented in Ref. 75, there is, however, no clear winner, with each method having its pro and cons. The numerical results we are going to discuss in Secs. IVE, V, and VI rely on the VLEM scheme outlined in Sec. IV B.

\section{Initialization of the VLEM code for oblique instabilities in a relativistic counterstreaming configuration}

In this article, we use the VLEM code to integrate the Vlasov-Maxwell equations in two spatial dimensions using two or three-momentum dimensions. That is, we use a $2 \mathrm{D} 2 \mathrm{~V}$ (or $2 \mathrm{D}$ ) and a 2D3V ( or $2 D \frac{1}{2}$ ) version of the code. Boundary conditions are periodic for space coordinates and open for momentum coordinates. The initial distribution function is chosen to be rapidly decaying with respect to the momentum coordinates to consider it to be zero at the boundaries in the momentum space.

Hereafter, we will indicate the numerical values of the physical quantities as expressed with respect to their normalization factors: time $t$, space coordinates $\boldsymbol{x}=(x, y)$, and momentum coordinates $\boldsymbol{p}$ are normalized to $\omega_{p}^{-1}, c \omega_{p}^{-1}$, and $m c$, respectively, and $\omega_{p e}=\sqrt{n_{0} e^{2} /\left(m_{e} \varepsilon_{0}\right)}$ is the electron plasma frequency for an initial electron population of density $n_{0}$. The electric $(\boldsymbol{E})$ and magnetic $(\boldsymbol{B})$ fields are normalized to $m \omega_{p} c / e$ and $m \omega_{p} / e$, respectively.

The beam-plasma system is chosen to consist of two hot electron beams with densities $n_{1}$ and $n_{2}$, and velocities $u_{1}^{(0)}=\beta_{1} c \geq 0$ and $u_{2}^{(0)}=\beta_{2} c \leq 0$ propagating in the $y$ - direction (cf. Fig. 1). Charge and current neutrality are initially ensured by requiring $n_{1}+n_{2}=n_{0}$ and $n_{1} \beta_{1}+n_{2} \beta_{2}=0$ at $t=0$. In order to deal with potentially relativistic thermal spreads, we choose to model the unperturbed electron beam/plasma system as a combination of drifting Maxwell-Jüttner distribution functions given by Eq. (16). In 3D, each distribution function takes the general form

$$
F_{0 j}(\boldsymbol{p})=\frac{\mu_{j}}{4 \pi \gamma_{j}^{2} K_{2}\left(\mu_{j} / \gamma_{j}\right)} \exp \left[-\mu_{j}\left(\gamma(\boldsymbol{p})-\beta_{j} p_{y}\right)\right],
$$

where the index $j=\{1,2\}$ labels the electron beams. Here, $\beta_{j}$ is the normalized drift velocity along $y, \gamma_{j}$ is the corresponding Lorentz factor, and $\mu_{j}=m c^{2} / k_{B} T_{j}$ is the normalized inverse temperature. $K_{2}$ is a modified Bessel function of the second kind and $k_{B}$ the Boltzmann constant. 
This configuration is, in principle, more general than that schematized in Fig. 1 and on which the numerical results we will discuss are based. In the 2DV2 geometry (which will be used in Sec. V C), the distribution function maintains an analogous expression of type $F_{0 j}\left(p_{x}, p_{y}\right)=A \exp \left[-\mu_{j}\left(\gamma\left(p_{x}, p_{y}\right)-\beta_{j} p_{y}\right)\right]$. In this case, the normalization constant $A$ is numerically computed by imposing $\int F_{0 j} d p_{x} d p_{y}=1$ for each species $j$.

In all simulations presented below, the initial distribution function is perturbed by introducing magnetic fluctuations on the $B_{z}$ component only. Indeed, in a geometry that just depends on two spatial coordinates (thus both in the $2 \mathrm{D} 2 \mathrm{~V}$ and $2 \mathrm{D} 3 \mathrm{~V}$ problems of interest here), the transverse magnetic (TM) components $\left(E_{x}, E_{y}, B_{z}\right)$ are decoupled from the transverse electric (TE) components $\left(E_{z}, B_{x}, B_{y}\right)$. The electromagnetic instabilities we are interested in just concern the TM components, and their (complex) phase is of the form $\sim e^{i\left(k_{x} x+k_{y} y-\omega t\right)+\eta t}$, where $\eta>0$ is the growth rate. In particular, two kinds of perturbations are used (where $\Delta k_{x}$ and $\Delta k_{y}$ are the fundamental wavenumber components) as follows:

- on a single mode,

$$
B_{z}=B_{0} \sin \left(n_{x} \Delta k_{x} x+n_{y} \Delta k_{y} y\right),
$$

- on a larger spectrum of modes

$$
B_{z}=\sum_{n_{x}, n_{y}=1}^{10} B_{0} \sin \left(n_{x} \Delta k_{x} x+n_{y} \Delta k_{y} y+\varphi_{n_{x}, n_{y}}\right),
$$

where $\varphi_{n_{x}, n_{y}}$ is a random phase. In principle, selecting an eigenmode of the Vlasov-Maxwell system would formally require to simultaneously perturb $f$ and all the relevant field components by respecting the appropriate phase and amplitude relations of that mode (we recall indeed that linearization of Faraday's equation gives $\boldsymbol{B}_{1}=(\boldsymbol{k} c / \omega)$ $\times \boldsymbol{E}_{1}$ ). In the $2 \mathrm{D}$ spatial geometry we consider here for TM modes, this would mean the need to include the perturbations on $E_{x}, E_{y}$, and $f$ since information on the frequency $\omega \neq 0$ of a time-resonant mode is not contained in Eqs. (25) and (26) alone. On the other hand, depending on the equilibrium distribution function, it can be not trivial to choose the appropriate form of the perturbation on $f$ that is compatible with the perturbation on the field modes. The practical experience we have with the VLEM solver indicates, however, that this is not really required for the problems studied here since, as we will see while discussing the nonlinear simulations, initializing the run with perturbations (25) or (26) allows the code to make emerge, after a transient time, a mode with the sought combination of wave numbers corresponding to the chosen values of $k_{x}=n_{x} \Delta k_{x}$ and $k_{y}=n_{y} \Delta k_{y}$. The possibility to select a single mode, to start the instability, is due to the completely noiseless character of the SL code, which does not add a background perturbation to the other eigenmodes into which perturbations (25) or (26), given only on one component of the eigenmo$\mathrm{de}(\mathrm{s})$, are decomposed during the initial transient time. [We mean with this that $(\partial f / \partial t)_{C G}=0$ exactly $^{66}$ at $t=0$ in the numerical implementation of Eq. (1).]

Finally, we note that, if the exact amplitude relations of the components of an eigenmode were provided by the initial perturbation, the noiseless character of the Vlasov code would, in principle, make it possible to start the instability with amplitudes of the perturbation close to the round-off errors of the computer.

\section{E. Conservations of the VLEM solver: Test case about the current filamentation instability}

The SL numerical scheme benefits of a high accuracy, which guarantees a very good description of wave-particle interactions, even in regions of the phase space where the density of particles is very low. In order to evidence these features, we reproduce here, as a test case, some numerical results that had been already discussed in Refs. 39 and 67 about the nonlinear dynamics of the CFI. Note that the numerical parameters of the CFI simulation we present in this section are the same as of most of the runs that have been performed for the OI case and which we will focus on later (but, of course, for the choice of the wave-vector components of the perturbation, which is here purely transverse, $\boldsymbol{k}=k_{x} \boldsymbol{e}_{x}$ ); therefore, the conservations we show here evidence the reliability of the numerical scheme also for the more complex case we will discuss next and for which we will show instead that the large amplitude fluctuations of $f$ play a role in the violation of entropy conservation that is not of numerical nature, differently from the numerical-induced CG, which (negligibly) concerns this CFI case. In addition to being useful to show the accuracy of the scheme in reproducing well established results, this test case is also useful for the interpretation of the nonlinear results we are going to discuss in Sec. V since it allows us to evidence the role played by the magnetic trapping process in the achievement of a metastable equilibrium for CFI, an instability that is "less complex" than the OI (for example, it is not time resonant and is essentially electromagnetic in nature).

We consider then a 2D2V geometry, and we initialize the system with the distribution of Eq. (24) for two symmetric beams with the same temperature $T_{1}=T_{2}=100 \mathrm{keV}$ (isotropic in the $x, y$-plane). The velocities of the two opposite beams are highly relativistic, $u_{1}^{(0)}=+0.9 c$ and $u_{2}^{(0)}=-0.9 c$, and equal densities are assumed to grant the symmetry of the configuration, $n_{1}=n_{2}=n_{0} / 2$. The other numerical parameters are a phase space sampling with $N_{x} N_{y}$ $\times N_{p_{x}} N_{p_{y}}=256^{2} \times 128^{2}$ points in a simulation box of dimensions $L_{x} \stackrel{p_{x}}{=} L_{y}=2 \pi c \omega_{p}^{-1},\left|p_{x} / m c\right| \leq 10$, and $\left|p_{y} / m c\right| \leq 10$ and a typical time step $\Delta t \omega_{p}=0.0025$. The initial perturbation is given as in (26) with a normalized amplitude $e B_{0} / m \omega_{p} \simeq 5 \times 10^{-3}$ and with $\Delta k_{x} d_{e}=\Delta k_{y} d_{e}=1$.

For this simulation, $N_{\text {threads }}=4$ (OpenMP) threads by processor, and a total number of processors $N_{\text {procs }}=256$ (i.e., $\sqrt{N_{\text {procs }}}$ for each spatial direction) have been used. This corresponds to a total of $N_{\text {threads }} \times N_{\text {procs }}=1024$ cores. The CPU time is close to $t_{C P U} \simeq 10.6 \mathrm{~h}$ for 50000 time steps, i.e., to $7.15 \times 10^{-10}$ s per "particle" and time step on each of the 1024 cores.

Figure 3 shows the typical energy transfer of CFI between the kinetic energy (bottom panel) and magnetic energy (top panel), which occurs while a quasi-static magnetic field is exponentially amplified at the expenses of the momentum anisotropy related to the two counterpropagating beams. ${ }^{24}$ The saturation arises due to the magnetic trapping mechanism. An oscillation of the different components of the energy, corresponding to the magnetic trapping bounce frequency, is also visible. Magnetic trapping provides here the dominant saturation mechanism of the CFI instability, which occurs when the current filaments reach a size of the order of the electron skin depth $d_{e}{ }^{45,46}$ Further details concerning the saturation mechanism of CFI can be found in Refs. 26, 29, 30, and 67, which are works exploiting the multi-stream model that is based on the invariance of the transverse canonical momentum. 

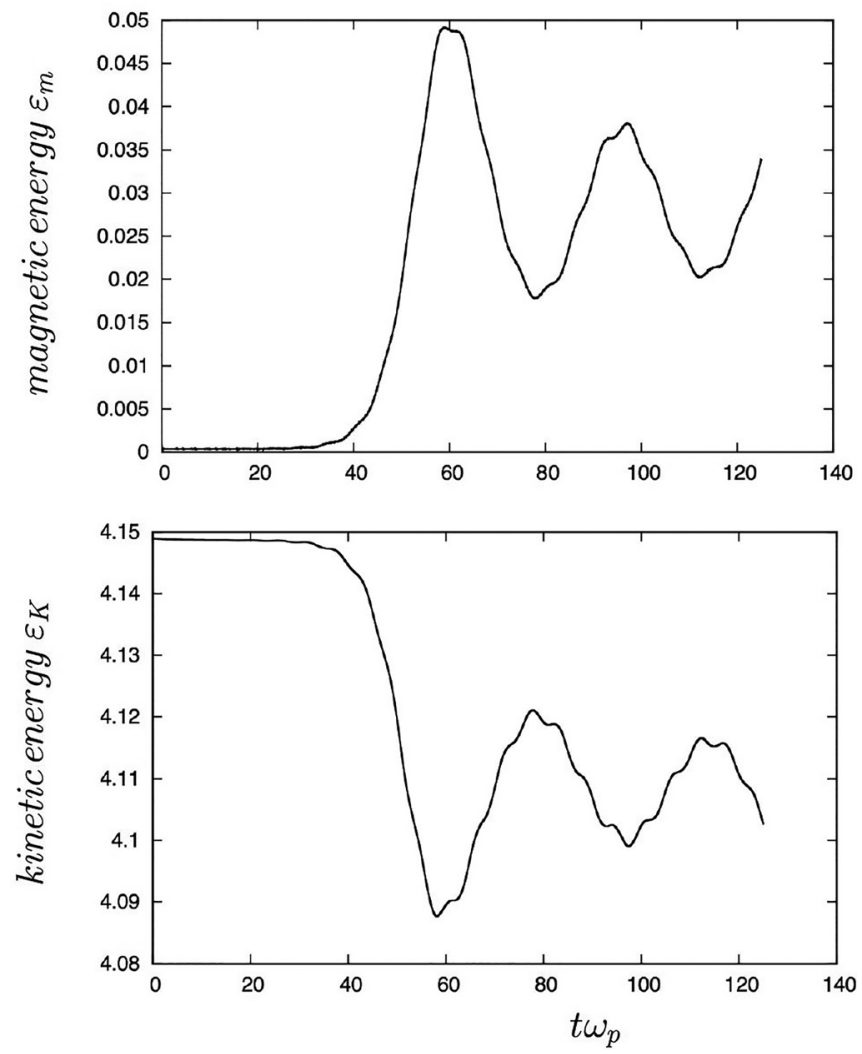

FIG. 3. Evolution of the magnetic (top frame) and kinetic (bottom frame) energies in the symmetric CFI with relativistic beam velocities $\left(\left|v_{1}\right|=\left|v_{2}\right|=0.9 \mathrm{c}\right)$ and temperatures $\left(T_{1}=T_{2}=100 \mathrm{keV}\right)$.

The time evolution of the total mass and energy is displayed in Fig. 4 on top and bottom panels, respectively. Mass and energy are very well conserved with a final (maximum) relative error of $1.5 \times 10^{-3}$ and $1.4 \times 10^{-2}$, respectively. The time evolution of the norm $L^{2}$, given by $\int\left(d^{2} x / V\right) \int f^{2} d^{2} p$, and the "Shannon" entropy (6) is shown in Fig. 5 on top and bottom panels, respectively. These quantities, too, are very well conserved: their relative variations are of the order of $5.5 \times 10^{-3}$ and $3 \times 10^{-3}$, respectively. It must be noted that the fluctuations of the distribution function remain small in amplitude and agree with the global conservation of the entropy: the small loss of conservation of all integral quantities shown here is entirely ascribable to a numerical induced $C G$.

\section{NUMERICAL SIMULATIONS OF ELECTROMAGNETIC LOW-FREQUENCY OBLIQUE MODES}

We now present and discuss simulation results of the nonlinear evolution of the OI for different choices of the initial perturbations given by Eqs. (25) and (26). The focus is on the impact that the lowfrequency branch of unstable modes denoted by symbol B in Fig. 2, which has been identified in paper $\mathrm{I}^{1}$ and which had not been evidenced by previous linear studies of the OI, has on the nonlinear evolution of these Weibel-type instabilities. We are going to show that these modes play a major role in wave-particle interactions because of their resonant character.
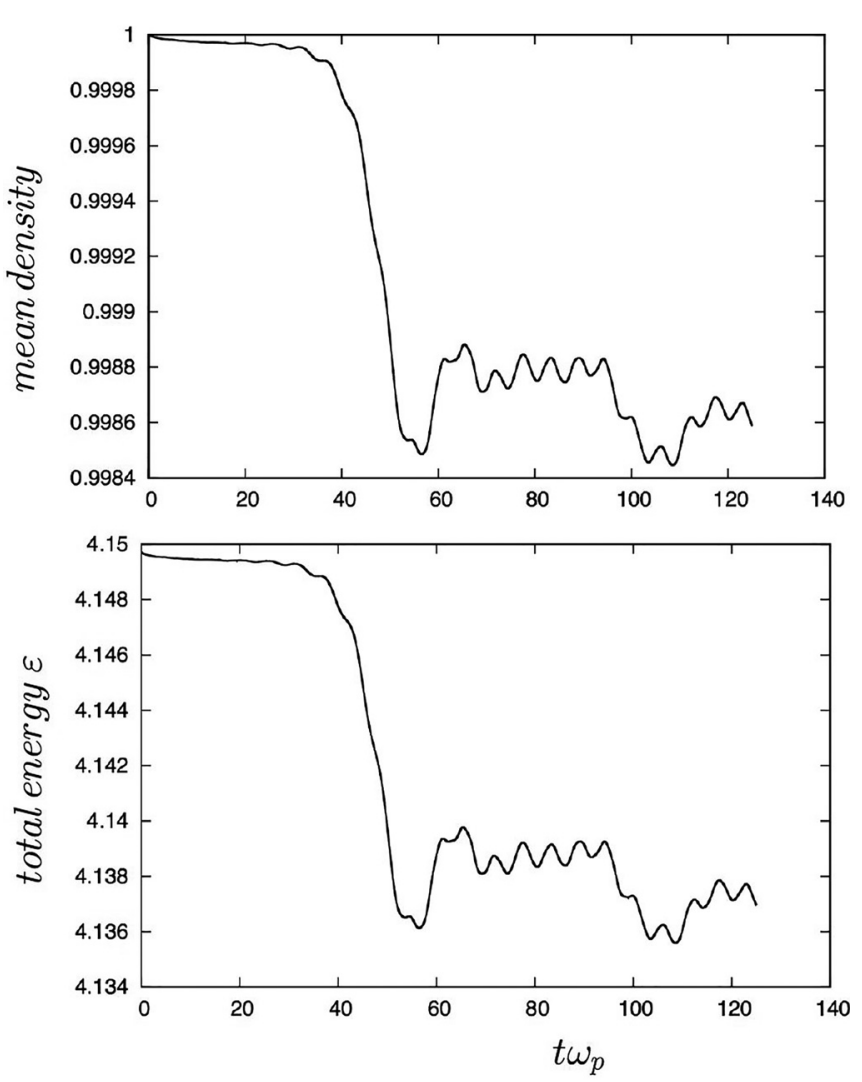

FIG. 4. Example of the accuracy of the VLEM code in modeling the CFI: time evolution of the mass (or the so-called mean density, top panel) and energy (bottom panel) vs time. The parameters of the simulation are the same as those of Fig. 3.

For this purpose, we have separately investigated the nonlinear dynamics of the two different branches of oblique solutions. In both cases, referred hereafter as cases $\mathrm{A}$ and $\mathrm{B}$, an initial value problem is considered, in which a single type of mode is perturbed, selected according to its wave numbers chosen by relying on the linear analysis summarized by Fig. 2: case A, discussed in Subsection VA, refers to the initial perturbation of the high-frequency mode, the so-called "standard" solution, while case B, discussed in Subsection V B, refers to the new branch of the linear dispersion studied in paper $\mathrm{I},{ }^{1}$ which corresponds to the low-frequency (small wave-number) set of unstable solutions. A nonlinear transition is observed from an $\mathrm{A} \rightarrow B$-dominated regime and from a low wavenumber to a large wavenumber Bdominated regime, respectively. Both transitions are accompanied by a magnetic trapping saturation scenario.

A third simulation case has been considered, in which both types of modes A and B have been simultaneously excited to investigate their mutual coupling during the evolution. This case is treated in Subsection V C.

Finally, another simulation case is discussed in Sec. VI, in which a large spectrum of B-type modes has been initially excited. In this case, a stochastic heating due to a spontaneous nonlinear transition from B-type, low-wavenumber modes to B-type, large-wavenumber modes is evidenced. This appears to provide a new mechanism of 

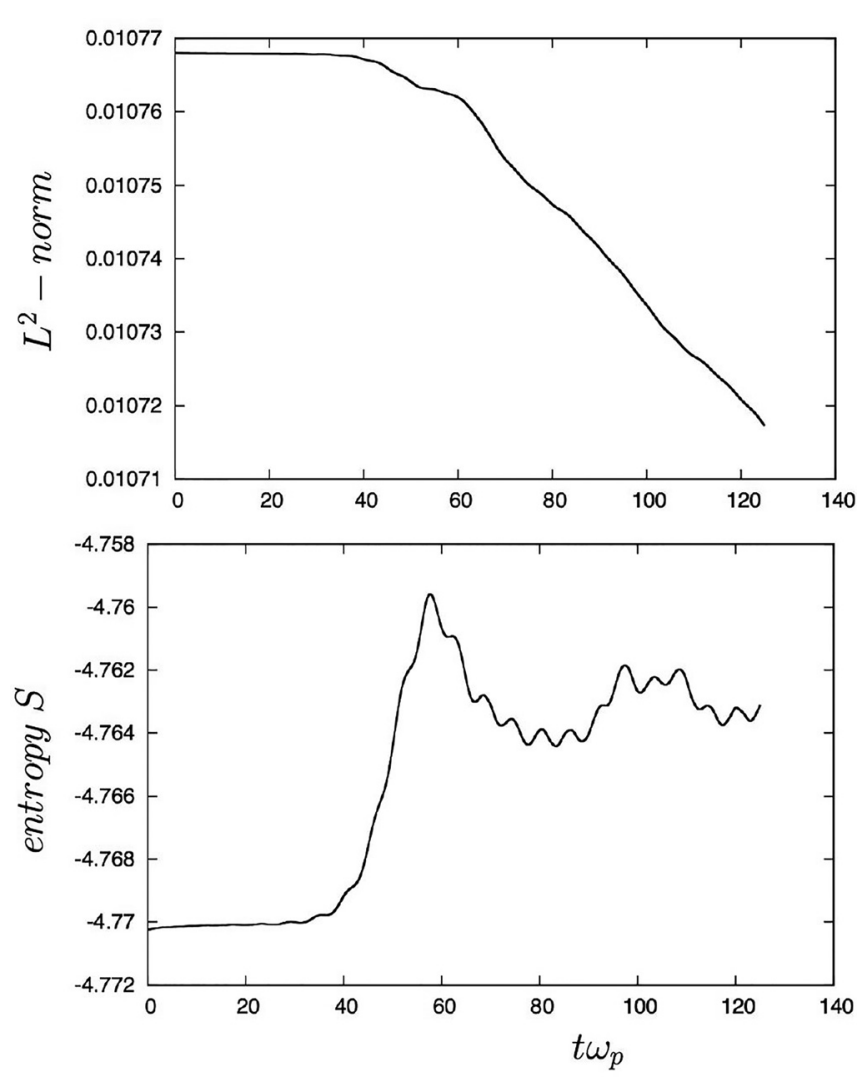

FIG. 5. Time evolution of the norm $L^{2}$ (top panel) and Shannon's entropy (bottom panel) for the CFI case of Figs. 3 and 4. An excellent conservation is measured.

saturation for Weibel-type modes, in the small wavelength regime, when $k d_{e} \gg 1$. The strong fluctuations of the distribution function, which characterize the nonlinear evolution of this simulation case, induce entropy variations different from those related to the numerical CG mechanism.

The essential features of the nonlinear simulations we have performed are summarized in Table I, where the nature of the instability, the regime investigated, and the observed saturation mechanism are summarized. The column "perturbation" specifies whether a magnetic perturbation is introduced on several modes, using (26), or only on a single mode $\left(n_{x}, n_{y}\right)$, using Eq. (25). In the figures that follow the version of the VLEM code $(2 \mathrm{D} 2 \mathrm{~V}$ or $2 \mathrm{D} 3 \mathrm{~V})$, which has been used to obtain them, is indicated in the caption.

\section{A. Case A: Nonlinear dynamics of high frequency modes}

A first numerical simulation has been performed in the case of mode A represented in Fig. 2 for a symmetric beam configuration. This simulation corresponds to a $2 \mathrm{D} 3 \mathrm{~V}$ (or $2 D \frac{1}{2}$ ) geometry, i.e., with three variables in the momentum space. In this geometry, we can make a direct comparison of the growth rates numerically obtained in the nonlinear simulation with the analytical results obtained by solving the full kinetic dispersion relation using a 3D Maxwell-Jüttner equilibrium.

We choose here to excite the modes $k_{x} d_{e}=2 \Delta k_{x} d_{e}=5$ and $k_{y} d_{e}=\Delta k_{y} d_{e}=2$ on the respective values $n_{x}=2$ and $n_{y}=1$ in wave numbers, which correspond to space box dimensions $L_{x} \simeq 2.513 d_{e}$ and $L_{y}=\pi d_{e}$.

Figures 6-11 show the results obtained for this numerical simulation. The latter was carried out by sampling the configuration space with $N_{x} N_{y}=128^{2}$ points and the momentum space with $N_{p_{x}} N_{p_{y}} N_{p_{z}}=32^{3}$ grid points, the time step is $\Delta t \omega_{p} \simeq 0.00375$, and the simulation was carried out for 40000 iterations in time. This simulation has a lower phase-space resolution than that of the CFI case discussed in Sec. IV C, but conservations are nevertheless very good (see Fig. 7).

The other physical parameters used in simulation are $T_{1}=T_{2}$ $=6 \mathrm{keV}, u_{1}^{(0)} \simeq+0.668 c$ and $u_{2}^{(0)} \simeq-0.668 c$, and $n_{1}=n_{2}=n_{0} / 2$. In order to excite the instability, a weak perturbation in the magnetic field component $B_{z}$ has been introduced in the form of Eq. (25), that is, $B_{z}=B_{0} \sin \left(k_{x} x+k_{y} y\right)$, with normalized amplitude $e B_{0} / m \omega_{p}$ $\simeq 0.05$.

Figure 6 shows the temporal evolution of the different energy components involved in the transfer mechanism: the kinetic energy $\epsilon_{K}$ on top, the magnetic energy $\epsilon_{m}$ in the middle, and the total electric energy $\epsilon_{e}$ on bottom (for the two components $E_{x}$ and $E_{y}$ of the electromagnetic field). In the first stage of the oblique instability, these quantities are very well conserved until time $t \omega_{p}=80$. Figure 7 shows the behavior of the total energy $\epsilon=\epsilon_{K}+\epsilon_{m}+\epsilon_{e}$ (on top) and entropy $S$ (on bottom) as a function of time. Note that here we have used the standard Shannon entropy definition of Eq. (6). Although a slow time evolution appears for the total energy, the latter remains well conserved throughout the simulation. Its maximum relative variation is $(0.3578-0.3571) / 0.3571 \simeq 2 \times 10^{-3}$. In contrast, the time evolution of entropy displays three distinct stages: after the initial equilibrium phase, the growth of the instability is accompanied by a strong and abrupt entropy increase, which is followed by a second phase of slower growth, once the instability has reached saturation. Both stages of entropy increase can be related to the numerical CG, with the

TABLE I. Different regimes of the simulations and corresponding nonlinear saturation mechanisms. Here, the letters LF and HF stand for low-frequency and high-frequency, respectively, while letters MT and ET stand for magnetic trapping and electrostatic trapping, respectively.

\begin{tabular}{|c|c|c|c|c|c|}
\hline Sections & Simulation & Perturbation & Instability & Regime & Saturation \\
\hline Section IV C & CFI & Several modes & Pure CFI & $k d_{e} \gtrsim 1$ & MT \\
\hline Section VA & Case $A$ & One mode & HF-OI & $k d_{e} \gtrsim 1$ & MT \\
\hline Section VB & Case $B$ & One mode & LF-OI & $k d_{e} \gtrsim 1$ & $\mathrm{MT}+\mathrm{ET}$ \\
\hline Section V C & $A+B$ & Both modes & Mode coupling & $k d_{e} \gtrsim 1$ & $\mathrm{MT}+\mathrm{ET}$ \\
\hline Section VI & Case $B$ & Large spectrum & Direct cascade & $k d_{e} \gg 1$ & ET \\
\hline
\end{tabular}



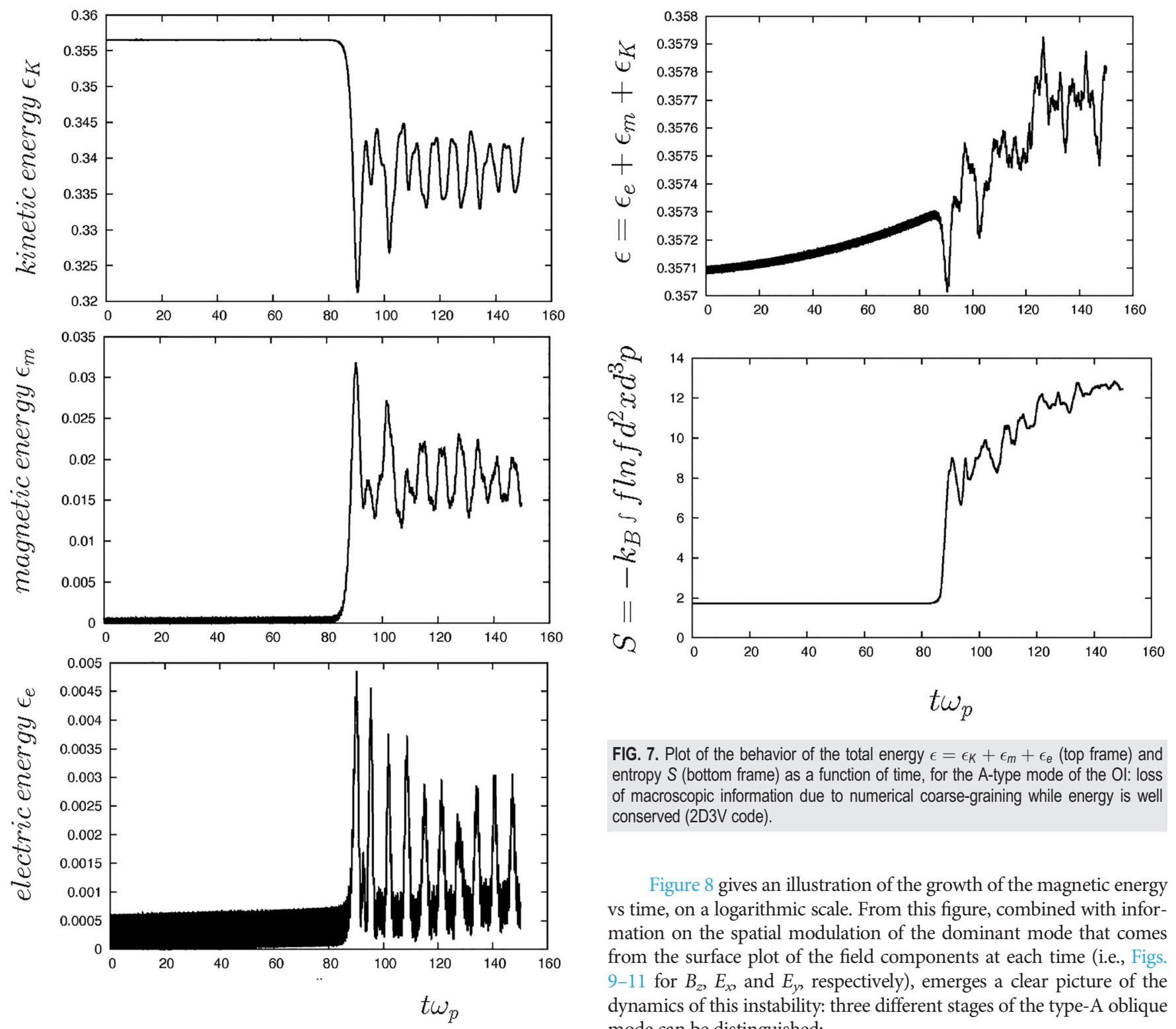

FIG. 6. Time evolution of different components involved in the energy transfer mechanism of the A-type mode of the Ol: the kinetic energy $\epsilon_{K}$ (on top), the magnetic energy $\epsilon_{m}$ (middle frame), and the total electrical energy $\epsilon_{\mathrm{e}}$ (on bottom). Oscillations at $\omega \simeq 0.492 \omega_{p}$, i.e., at about half the magnetic bounce frequency, are visible after saturation, indicating the combined effect of magnetic and electrostatic trapping related to the $\mathrm{A} \rightarrow \mathrm{B}$ mode transition (2D3V code).

different rates of entropy production being due to the different rates at which phase-space filamentation occurs during the growth and the saturation stage of the instability. The saturation of the growth of entropy, which seems to be approached at the end of the simulation for $t \omega_{p} \gtrsim 135$ (Fig. 7, bottom panel), indicates that a metaequilibrium state is approached, in which filamentation in the velocity space is not developing any further below the size of the elementary cell.

FIG. 7. Plot of the behavior of the total energy $\epsilon=\epsilon_{K}+\epsilon_{m}+\epsilon_{\theta}$ (top frame) and entropy $S$ (bottom frame) as a function of time, for the A-type mode of the Ol: loss of macroscopic information due to numerical coarse-graining while energy is well conserved (2D3V code).

Figure 8 gives an illustration of the growth of the magnetic energy vs time, on a logarithmic scale. From this figure, combined with information on the spatial modulation of the dominant mode that comes from the surface plot of the field components at each time (i.e., Figs. 9-11 for $B_{z}, E_{x}$, and $E_{y}$, respectively), emerges a clear picture of the dynamics of this instability: three different stages of the type-A oblique mode can be distinguished:

(1) The first stage is observed for $t \omega_{p} \leq 80$, where the contribution from the magnetic energy remains small. During this phase, the Maxwell-Jüttner equilibrium (which is here very close to the classical Maxwell-Boltzmann equilibrium) displays magnetic fluctuations in times that are close to the inverse of the electromagnetic wave frequency $\omega=\sqrt{\omega_{p}^{2}+k^{2} c^{2}} \simeq 5.4 \omega_{p}$. In this time interval, a high frequency component with $2 \omega-10 \omega_{p}$, which is well above the plasma frequency, can be indeed recognized: this mode is not amplified in time since it corresponds to spurious oscillations that appear in a transient time interval because of the fact that the initial perturbation with wave numbers $k_{x} d_{e}=5$ and $k_{y} d_{e}=2$ was given only on the $B_{z}$ component (cf. Sec. IV B).

(2) The second stage, in the interval $80 \leq t \omega_{p} \leq 90$, starts when the energy of the initial perturbation has been redistributed 


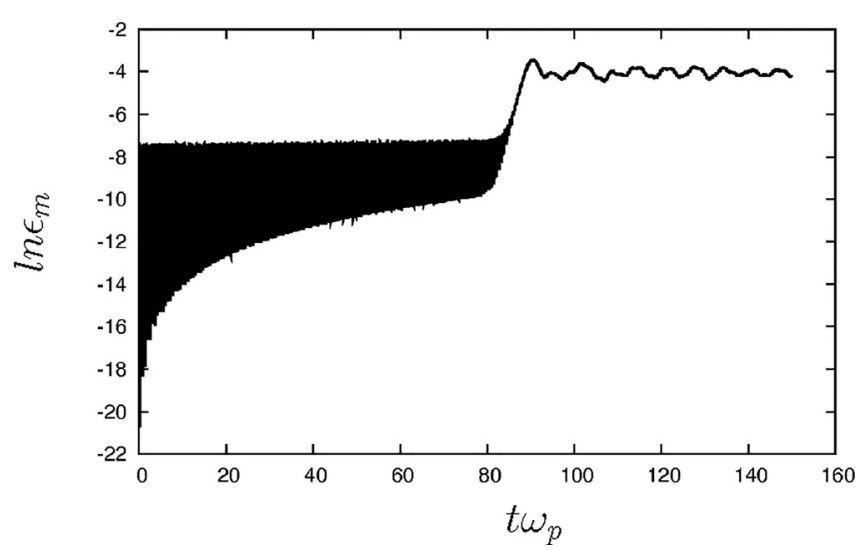

FIG. 8. Plot of the magnetic energy, on a logarithmic scale, as a function of time. The figure shows clearly the three different steps of the instability: the transient stage (characterized by high frequency oscillations due to the presence of high frequency modes), an exponential growth (of a low-frequency mode), and then the saturation phase. The simulation corresponds to case $A$ (2D3V code).

among the eigenmodes of the system so that a larger amplitude mode with $k_{x} d_{e}=5$ and $k_{y} d_{e}=2$ has been "properly build": the transient phase has ended, and the linear growth of the high frequency mode that we wanted to excite starts. Its growth rate $\eta / \omega_{p}=0.35$, measured from the simulation, agrees well with the theoretical value $\eta / \omega_{p}=0.34$ of the linear analysis, thus evidencing that the selected A-type mode has been efficiently excited (the evolution of the corresponding mode can also be followed in the plots of fields). Note that, because of the perturbation initially selected, the modes $n_{x}=1, n_{y}=0$ (i.e., the $\mathrm{CFI}$ ), and $n_{x}=1, n_{y}=1$ (i.e., a lower frequency oblique mode in the region where solutions A and B overlap) and, for example, the modes $n_{x}=2, n_{y}=1$ (i.e., another A-type oblique mode), and $n_{x}=0, n_{y}=1$ (the TSI) are also present. Their contribution, like that of the other possible eigenmodes, at this stage appears, however, negligible. This can be deduced from the growth rate and from other cross-related information: the emergence of the initially perturbed mode with $n_{x}=2$ and $n_{y}$ $=1$ is clearly visible already at $t=82.5$ in the surface plot of $B_{z}$ (Fig. 9, top panel), and differently from the CFI, it displays a strong electrostatic component, as can be seen from the space modulation of the contour-plot of $E_{x}$ (in Fig. 10) and $E_{y}$ (Fig. 11).

(3) The third stage, corresponding to the nonlinear saturation of the $n_{x}=2, n_{y}=1$ mode, is visible for $t \omega_{p} \geq 90$. In this stage, a spatial modulation with $n_{x}=1, n_{y}=1$, that is, $k_{z}=2.5$ and $k_{y}=2$, clearly emerges: it can be recognized, for example, in the profiles of $B_{z}$ (see the bottom panel Fig. 9). This, combined with the fact that the electric energy (Fig. 6, on the bottom frame) exhibits an oscillating behavior at a low frequency close to the value $\omega_{O I} \simeq 0.492 \omega_{p}$, i.e., at a frequency well below the plasma frequency or the magnetic bounce frequency (see below), indicates that a nonlinear transition has occurred from the known, initially dominant solution [the high-frequency mode $\left.\left(n_{x}=2, n_{y}=1\right)\right]$ to a low-frequency solution, which belongs to the class or type-B roots (cf. Fig. 2). The latter is found at a
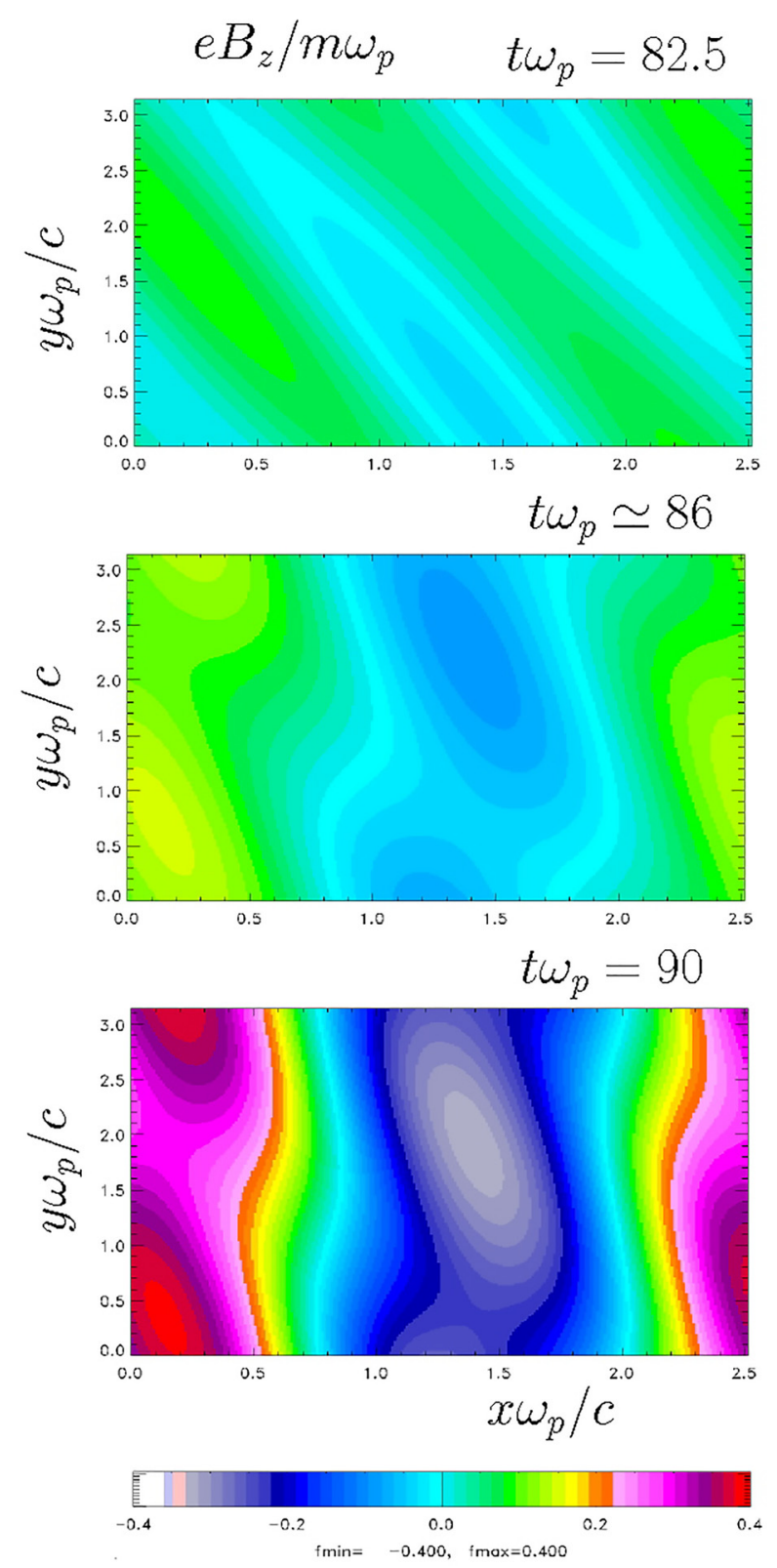

FIG. 9. Plot of the normalized amplitude of the magnetic field component $B_{z}$ at three instants, during the growth of the A-type mode of the Ol: the upper and middle frames correspond to the linear growth of the initially excited oblique mode $\left(n_{x}, n_{y}\right)=(2,1)$, and the bottom frame shows the magnetic field at saturation (2D3V code).

frequency smaller than the frequency of the linear mode OI. This indicates a nonlinear shift induced by an electrostatic-type trapping, a trapping mechanism different from the standard magnetic trapping usually expected at saturation of Weibeltype instabilities.

Note that the couple of values $k_{x}=2.5, k_{y}=2$ lie in the region of the $\left(k_{x}, k_{y}\right)$ plane where both A and B solutions overlap (Fig. 2). In 

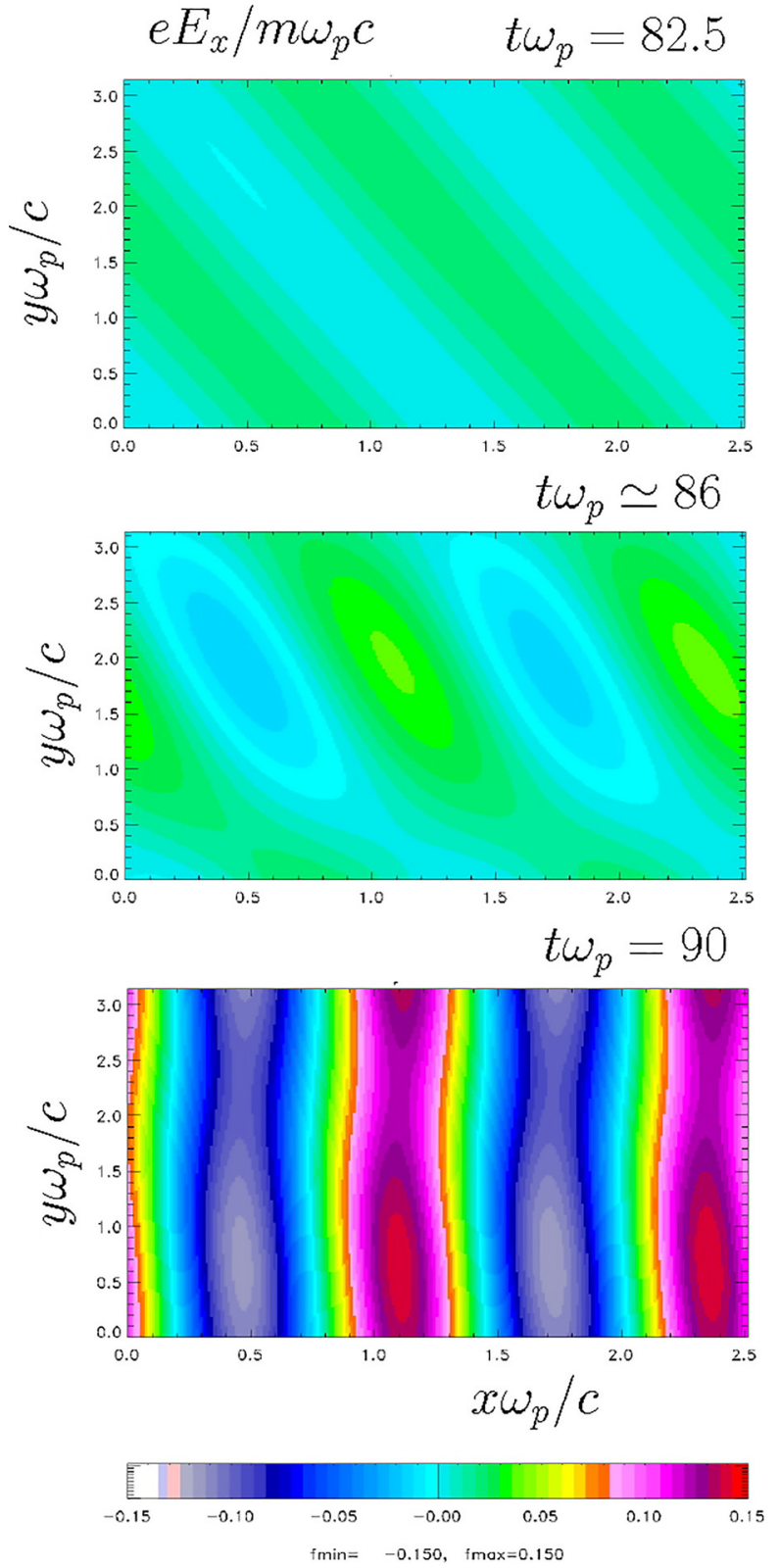

FIG. 10. Plot of the normalized amplitude of $E_{x}$ for the simulation case $A$ of the OI. The times are the same as those of Fig. 9. The strongly electrostatic nature of the mode is here evidenced, and the spatial oscillations related to the second harmonic of the initial $\left(n_{x}, n_{y}\right)=(2,1)$ mode are visible (2D $3 \mathrm{~V}$ code).

this third stage that corresponds to $k d_{e} \sim 3$, i.e., to "large" wavenumbers, the saturation mechanism is due to magnetic trapping. Magnetic trapping structures are visible as "magnetic vortices" in the contour-plot of $B_{z}$, in the bottom panel of Fig. 9. The magnetic bounce frequency $\Omega_{b}$ is estimated to be

$$
\frac{\Omega_{b}}{\omega_{p}}=\sqrt{\frac{|\boldsymbol{k}| c}{\omega_{p}} \frac{p_{1}}{m c} \frac{e B_{\max }}{m \omega_{p}}} \simeq \sqrt{3 \times 0.9 \times 0.3} \simeq 0.9 \sim \frac{2 \omega_{O I}}{\omega_{p}} .
$$
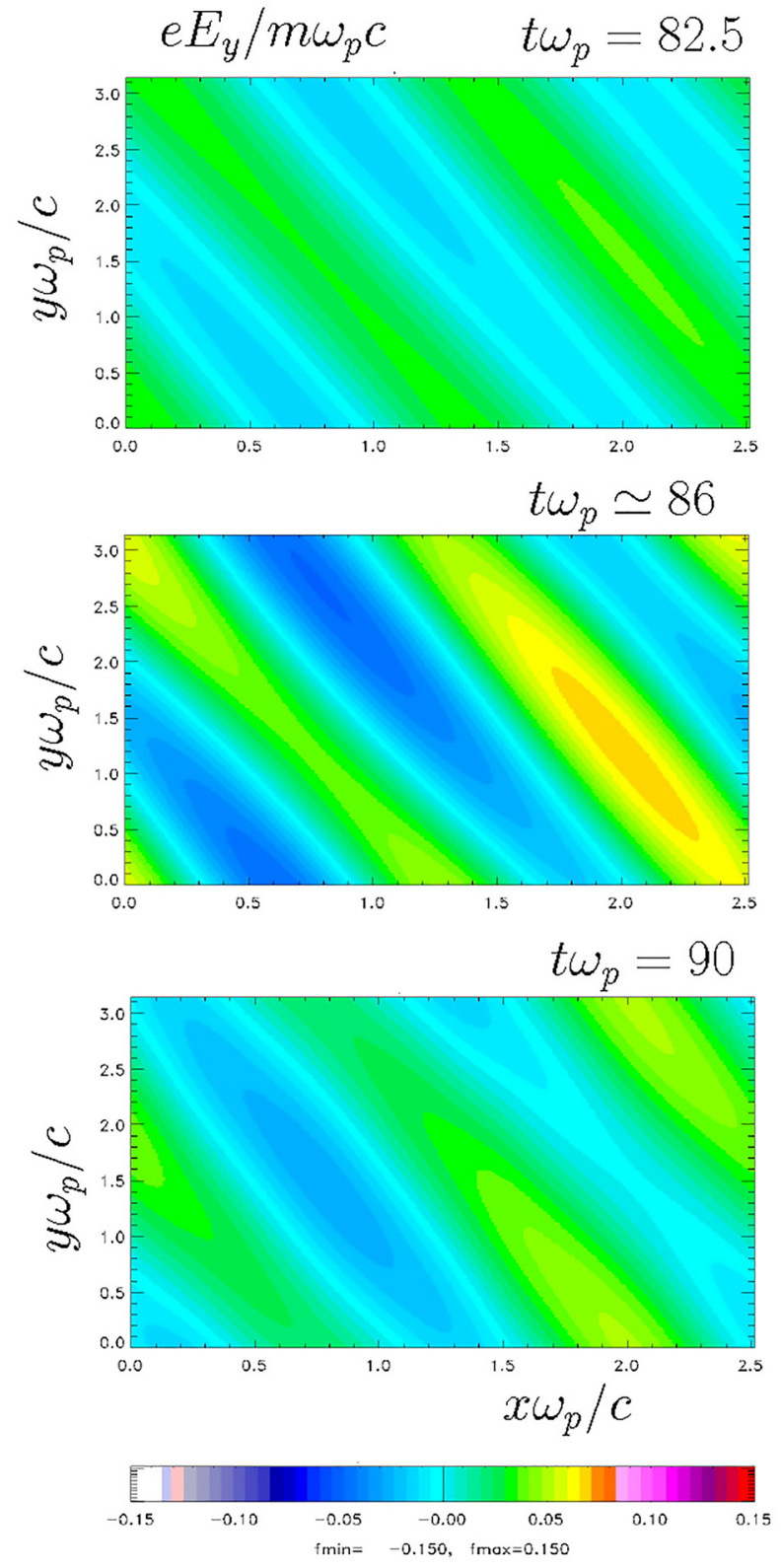

FIG. 11. Plot of the normalized amplitude of $E_{y}$ for the simulation case $A$ of the OI. The times are the same as those of Figs. 9 and 10 (2D3V code).

Oscillations at this frequency can be observed just after saturation in the time evolution of the magnetic energy component (middle panel of Fig. 6). Note the presence of the harmonic $n_{x}=2$ in both $E_{x}$ (Fig. 10) and $E_{y}$ components (Fig. 11).

In summary, we have observed the transition from the linear growth of a dominant high-frequency mode with $n_{x}=2$ and $n_{y}=1$ (type A solution) to a nonlinear regime dominated by a lower frequency $n_{x}=1$ and $n_{y}=1$ mode, compatible with a B-type solution. Contributions to the evolution coming also from CFI-type modes are present, due to the type of initialization we have chosen, but are not 
dominant in the linear stage. Saturation due to magnetic trapping occurs, however, like for the CFI instability. Here, it takes place at $k d_{e} \sim 1-3$ and induces a kinetic plasma heating related to the formation of phase-space magnetic vortices, not shown here (see, in this regard, also case B and related Fig. 14, next).

\section{B. Case B: Nonlinear dynamics of low frequency modes}

We now consider the nonlinear evolution of a type- $B$ "low" frequency oblique mode. Most of the numerical parameters are the same of the simulation of Case $A$. The box lengths have been adjusted to $L_{x}=4.0879 d_{e}$ and $L_{y}=2.513274 d_{e}$, which correspond to the fundamental modes $\Delta k_{x}=2 \pi / L_{x} \simeq 1.537$ and $\Delta k_{y}=2 \pi / L_{y}=2.5$. In order to excite the mode $k_{x} d_{e} \simeq 3.07$ and $k_{y} d_{e}=5$ (at the center of the lobe B, cf. Fig. 2), we have introduced an initial perturbation on $B_{z}$ using Eq. (25), with wave numbers $n_{x}=2, n_{y}=2$ and with a normalized amplitude $e B_{0} / m \omega_{p} \simeq 0.05$. The density, temperature, and modulus of the fluid momentum are identical for both beams: $n_{1}=n_{2}=n_{0} / 2, T_{1}=T_{2}=6 \mathrm{keV}, u_{1}^{(0)}=+0.9 c$, and $u_{2}^{(0)}=-0.9$.
The numerical simulation was performed by using a $2 D \frac{1}{2}$ version of the VLEM solver with more than 40000 iterations and a normalized time step $\Delta t \omega_{p}=0.00375$.

The results are shown in Figs. 12-14.

Figure 12 shows the temporal evolution of integral quantities: the relativistic kinetic energy $\epsilon_{K}$ (top left), the magnetic energy $\epsilon_{m}$ (top right), the total electric energy $\epsilon_{e}$ (bottom left), and Shannon's entropy $S$ (bottom right). The behavior of these quantities is qualitatively analogous to that already described for case A. The asymptotic metastable state characterized by the conservation of the entropy, not affected anymore by the numerical coarse graining, is here reached at time $t \omega_{p} \sim 120$. This follows a saturation regime dominated by the electrostatic trapping, as we will see, which is evidenced by the time evolution of the electric energy $\epsilon_{e}$ shown in Fig. 12 (at left bottom), while Fig. 13 displays the dynamics of the different steps of the OI by considering the behavior of the magnetic field $B_{z}$. Also in this simulation, three stages of the evolution of the instability can be recognized, although they are quantitatively different than before because of the different nature of the mode driving the linear instability
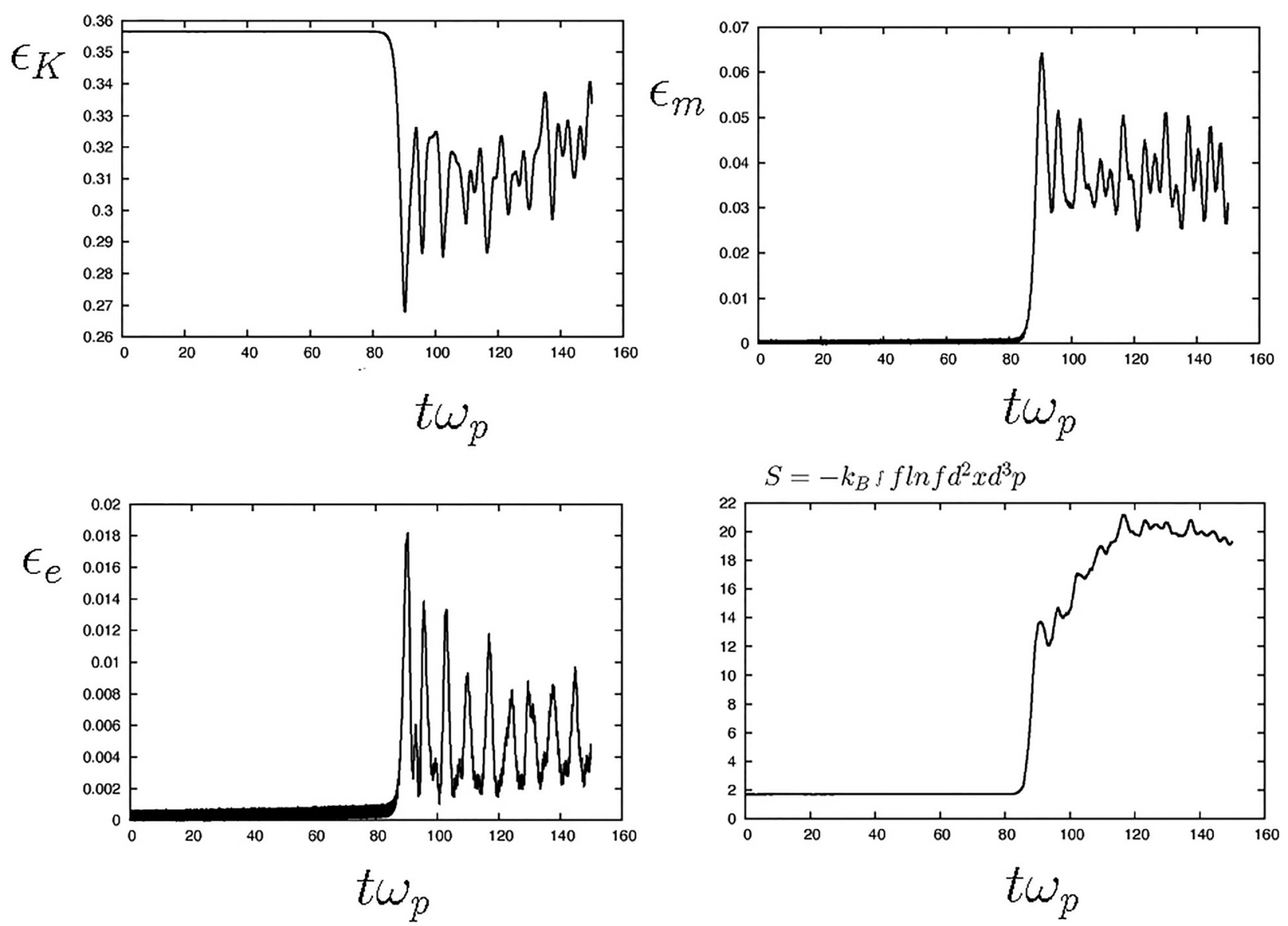

FIG. 12. Time evolution of the relativistic kinetic energy $\epsilon_{K}$ (top left panel), the magnetic energy $\epsilon_{m}$ (top right), the total electric energy $\epsilon_{e}$ (bottom left), and the entropy $S$ (bottom right). These results refer to the simulation case of a low-frequency $B$-type mode of the Ol, excited with a magnetic perturbation on $k_{x} d_{e} \simeq 3.07$ and $k_{y} d_{e}=5$. Electrostatic trapping is the dominant process at saturation, as evidenced by the time oscillations of $\epsilon_{e}$ after $t \omega_{p} \simeq 90$ (bottom left panel) (2D3V code). 

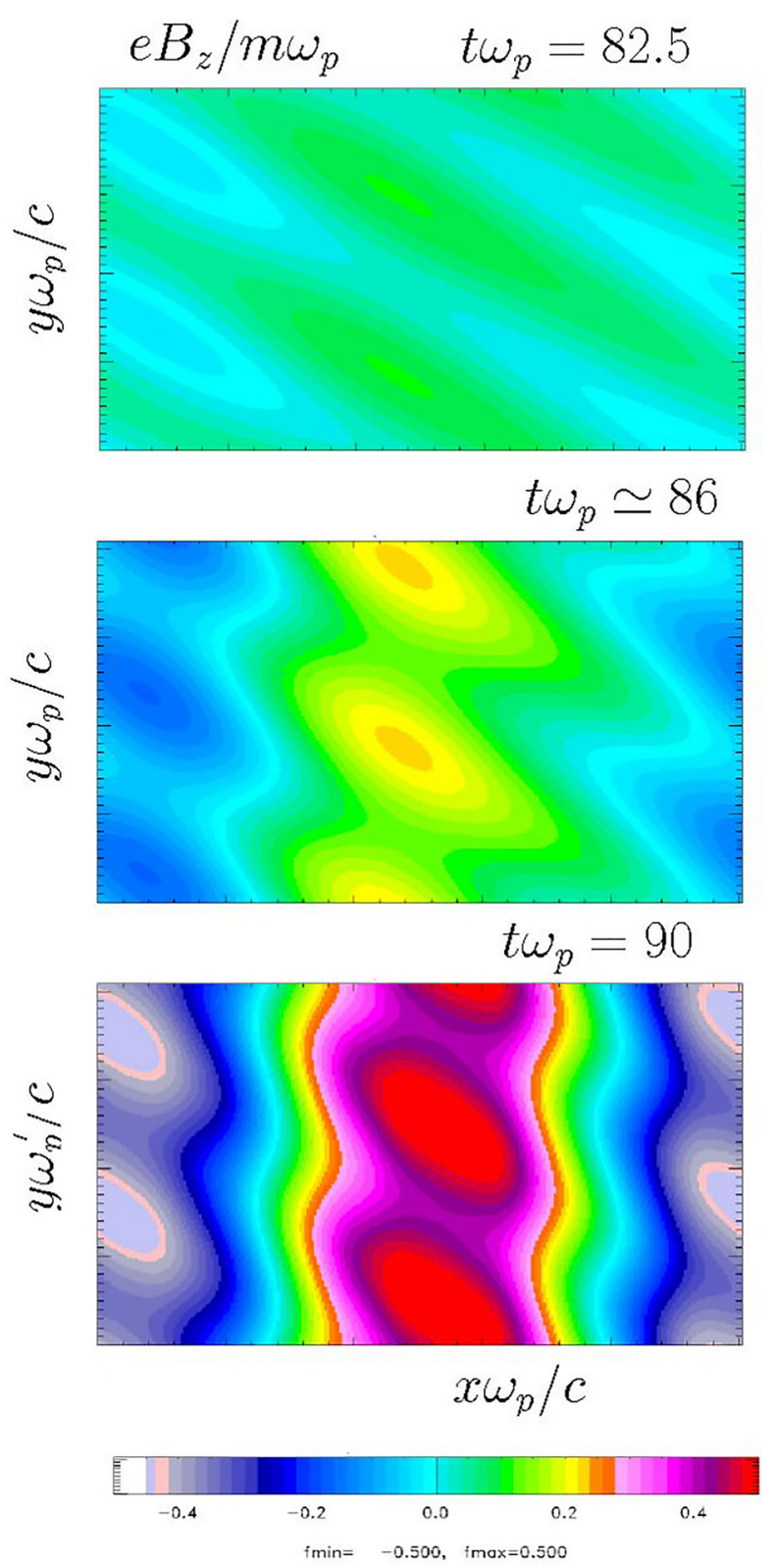

FIG. 13. Plot of the magnetic field $B_{z}$ at three different times of the $B$-case simulation of the Ol: during the linear regime of the oblique instability (top panel), at the maximum of the exponential growth (middle panel), and at the saturation of the OI (bottom panel) (2D3V code).

(1) The transient stage has essentially the same temporal extension of case A: it lasts until t $\omega_{p} \lesssim 80$, as it is visible from Fig. 12 .

(2) The emergence and exponential amplification (as expected in the linear regime) of the low frequency mode $n_{x}=2, n_{y}=2$ is clearly visible for $80 \lesssim t \omega_{p} \lesssim 90$ from the evolution of $\epsilon_{m}$ (Fig. 12) and from the profile of $B_{z}$ at $t \omega_{p} \simeq 82.5$ (Fig. 13, first panel): the value of its growth rate, numerically found to be $\eta_{\text {num }} / \omega_{p} \simeq 0.35$, remains close to the value of case $\mathrm{A}$ and agrees with the linear analysis.
(3) Saturation of the linear growth is achieved at $t \omega_{p} \simeq 90$, and the nonlinear saturated regime characterizes the time interval $t \omega_{p} \geq 90$. The emergence of a nonlinear shift in the wavenumber with $\left(n_{x}=1, n_{y}=2\right)$ becomes manifest in the profile of $B_{z}$ at $t \omega_{p} \simeq 90$ (Fig. 13, bottom panel). Magnetic vortices, which usually characterize the magnetic trapping saturation regime of CFI, are also present since $t \omega_{p} \simeq 86$ : as the CFI has $k_{y}=0$, their spatial modulation in the $x$ direction is due to the contribution of the oblique mode $\left(n_{x}=1, n_{y}=2\right)$. However, the magnetic trapping cannot explain the oscillatory behavior observed in the nonlinear stage of the OI after saturation.

The electrostatic bounce frequency is close to

$$
\frac{\omega_{b}}{\omega_{p}}=\sqrt{\frac{|\boldsymbol{k}| c}{\omega_{p}} \frac{e|\boldsymbol{E}|}{m \omega_{p} c}} \sim \sqrt{5 \times 0.05}=0.5,
$$

which is found to be in good agreement with the numerical value of $\omega \simeq 0.46 \omega_{p}$, while the corresponding magnetic bounce frequency can be estimated to be

$$
\frac{\Omega_{b}}{\omega_{p}}=\sqrt{\frac{|\boldsymbol{k}| c}{\omega_{p}} \frac{p_{1}}{m c} \frac{e B_{\max }}{m \omega_{p}}} \simeq \sqrt{5.22 \times 0.9 \times 0.4} \simeq 1.37,
$$

a value larger than $\omega_{p}$. Furthermore, the saturation related to a particle trapping regime of electrostatic nature, which here we encounter for a final state with a dominant $k d_{e} \simeq 5$ mode, is confirmed by the $x$ - $p_{x}$ dependence of the electron distribution function shown in Fig. 14 at different times: the characteristic trapping structures (labeled with "ET" in the figure) are shown to grow since the linear stage up to saturation, together with magnetic trapping structures (evidenced as "MT," in the figure). Finally, note that the saturated state is here characterized (similar to the previous case A) by a global kinetic heating of the plasma: this is due to the large spreading of the distribution function along the $p_{x}$ coordinate (Fig. 14, bottom panels). This "heating" process, however, is not stochastic but driven by the emergence of coherent vortices in the phase space, which are associated with both the electrostatic and magnetic trapping saturation mechanisms.

In summary, we have seen that exciting a magnetic perturbation spatially modulated with wave-numbers $\left(n_{x}=2, n_{y}=2\right)$, that corresponds to a low-frequency type-B solution of the oblique instability, leads to a different saturation process with respect to the previous case of $A \rightarrow B$ transition: in the present case, although magnetic trapping is still present, particle trapping in the electrostatic potential wells dominates. A kinetic heating process due to the formation of phasespace vortices related to the electric trapping at $k d_{e} \sim 5$ characterizes this nonlinear dynamics.

\section{Simultaneous initial excitation of both low and high frequency oblique modes}

Let us now discuss a simulation in which both linear solutions A and $\mathrm{B}$ have been simultaneously excited. For this purpose, we have used a 2D2V version of the VLEM solver, which allows us to considerably increase the sampling of the distribution function in the momentum space.

We have modified the lengths of the system (here $L_{x}=L_{y}=2 \pi d_{e}$ ) in order to be able to excite both types of solutions 

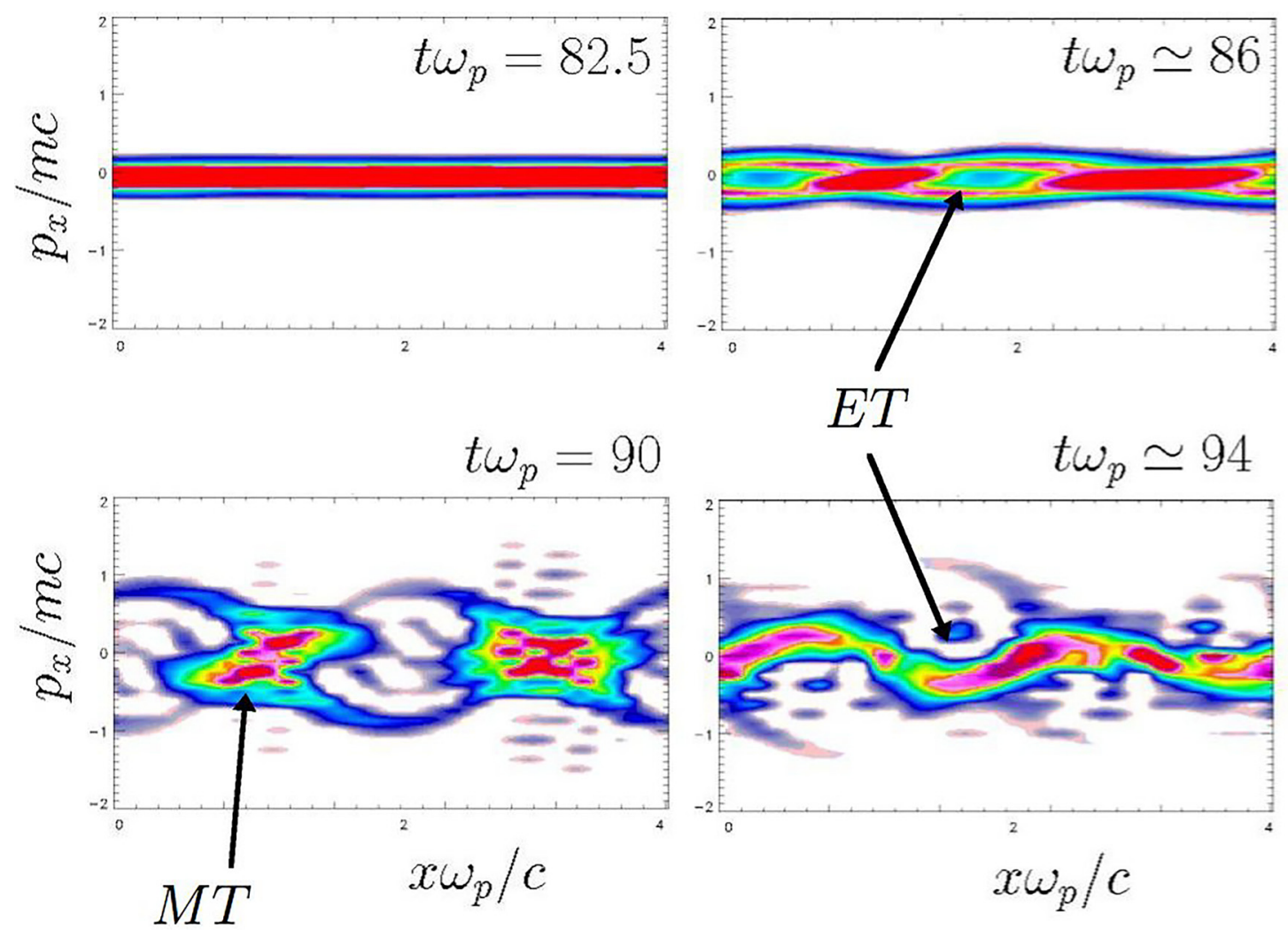

FIG. 14. Representation of the distribution function in the $x-p_{x}$ phase space at four different times during the $B$-case simulation. The formation and growth of both magnetic trapping (MT) and electrostatic trapping (ET) structures at saturation is evidenced are arrows (2D3V code).

from the same term of magnetic perturbation. This is chosen in the form of a sum of perturbations of the kind of Eq. (25),

$$
B_{z}=B_{0} \sin \left(5 \Delta k_{x} x+2 \Delta k_{y} y\right)+B_{0} \sin \left(3 \Delta k_{x} x+5 \Delta k_{y} y\right) .
$$

The normalized amplitude of the magnetic field is chosen to be smaller than before, $e B_{0} / m \omega_{p}=0.005$. A sampling of $N_{x} N_{y}=256^{2}$ and $N_{p_{x}} N_{p_{y}}=128^{2}$ points has been used. The simulation required 50000 time iterations, with a time step $\Delta t \omega_{p}=0.0025$. The results are summarized in Figs. 15-21.

Figure 15 shows the evolution over time of the contributions to energies: the kinetic energy $\epsilon_{K}$ on the top panel, the magnetic energy $\epsilon_{m}$ in the middle panel, and finally the total electric energy $\epsilon_{e}$ in the bottom panel. The conversion of kinetic energy into magnetic energy, which is characteristic of the oblique mode in the linear phase, is clearly observed. Due to the mixed electromagnetic-electrostatic character of the oblique modes excited, the electrostatic energy also grows together with the electromagnetic component (lower panel of Fig. 15). The qualitative behavior of the three stages (transient, linear stage, and nonlinear saturation) discussed before for cases A and B is recovered. As visible from Fig. 17, the electrostatic component is relatively small. However, it plays a major role in the saturation mechanism of the OI. The nonlinear stage, close to saturation, clearly shows the emergence of a low frequency mode characterized by the appearance of strong oscillations at a frequency smaller than the plasma frequency.
Although small in amplitude in comparison to the magnetic energy, the electric energy exhibits strong oscillations, after saturation, at a frequency close to $\omega \simeq 0.48 \omega_{p}$.

In order to analyze in greater detail the initial phase of the nonlinear stage of the instability and in particular the coupling mechanism of the two low-frequency and high-frequency oblique modes, the evolution of the energy components is reproduced in Fig. 16 on a logarithmic scale: a beating mechanism between the two oblique modes appears evident since the transient time in the magnetic energy $\epsilon_{m}$ and in the two components $\epsilon_{e, x}$ and $\epsilon_{e, y}$ of the electric energy (for convenience, the magnetic energy contribution $\epsilon_{m}$ has been shown alone, in the top frame). The overall energy balance $\epsilon=\epsilon_{K}+\epsilon_{m}+\epsilon_{e, x}+\epsilon_{e, y}$ shown in Fig. 17 evidences the excellent total energy conservation (dash-dotted line), whose relative variation is about $7 \times 10^{-3}$ by the end of the simulation, as well as the small fraction of kinetic energy, which is transferred to the electrostatic components.

The conservation of other two integral invariants, namely, the total "mass" $\bar{n}=\int\left(d^{2} x / V\right) \int f d^{2} p$ and the $L^{2}-$ norm $\int\left(d^{2} x / V\right) \int|f|^{2} d^{2} p$, is shown in Fig. 18. The maximum relative variation of the former is about $10^{-4}$, while it is larger for the second, being about $10 \%$. This imperfect conservation is due to the finite size of the interval in the momentum coordinates and to its sampling and must be compared with the $18 \%$ total relative variation of about previous $2 \mathrm{D} 3 \mathrm{~V}$ simulations of cases $\mathrm{A}$ and $\mathrm{B}$. In the present $2 \mathrm{D} 2 \mathrm{~V}$ simulation, 

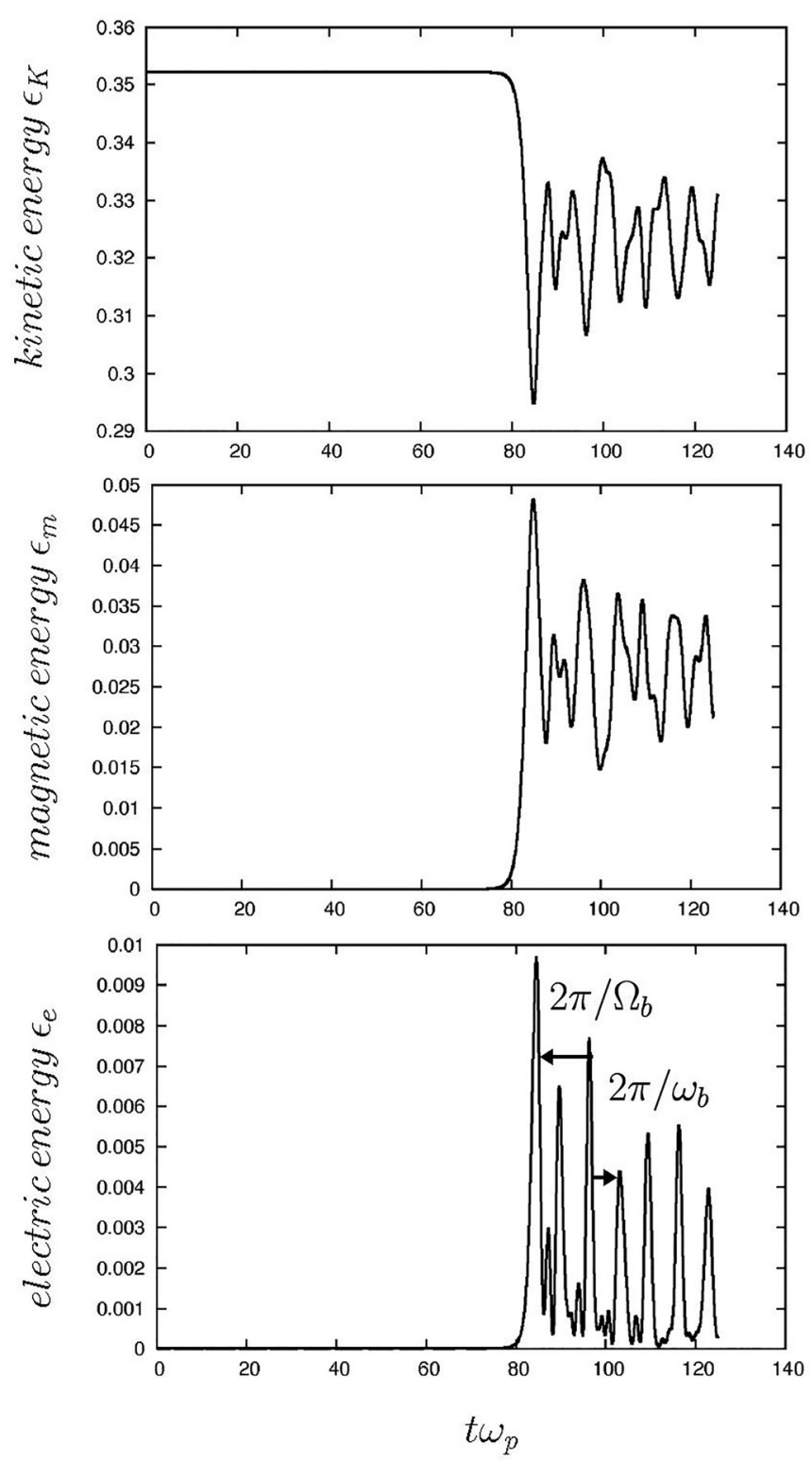

FIG. 15. Time evolution of the kinetic energy $\epsilon_{K}$ (top panel), the magnetic energy $\epsilon_{m}$ (middle panel), and the total electric energy $\epsilon_{e}$ (bottom panel) for the simulation case of simultaneous excitation of modes $A$ and $B$. The conversion of kinetic energy to magnetic energy is clearly observed, as expected in the case of the oblique instability. The magnetic bounce period $\left(2 \pi / \Omega_{b}\right)$ and the electrostatic bounce period $\left(2 \pi / \omega_{b}\right)$ have been explicitly indicated in the bottom frame (2D2V code).

the sampling of the phase-space domain has been indeed increased by a factor 4 for the space coordinate and by a factor 16 for the relevant momentum coordinate with respect to previous $2 \mathrm{D} 3 \mathrm{~V}$ simulations, thus also indicating a good convergence of the scheme.

The result of the beating of the two excited modes is visible in the contour plots of the fields, reproduced in Fig. 19: the two top frames show the evolution of the $B_{z}$ component of the magnetic field at two different times. The mode is excited on the wavevector $k_{x} d_{e}=2$ and
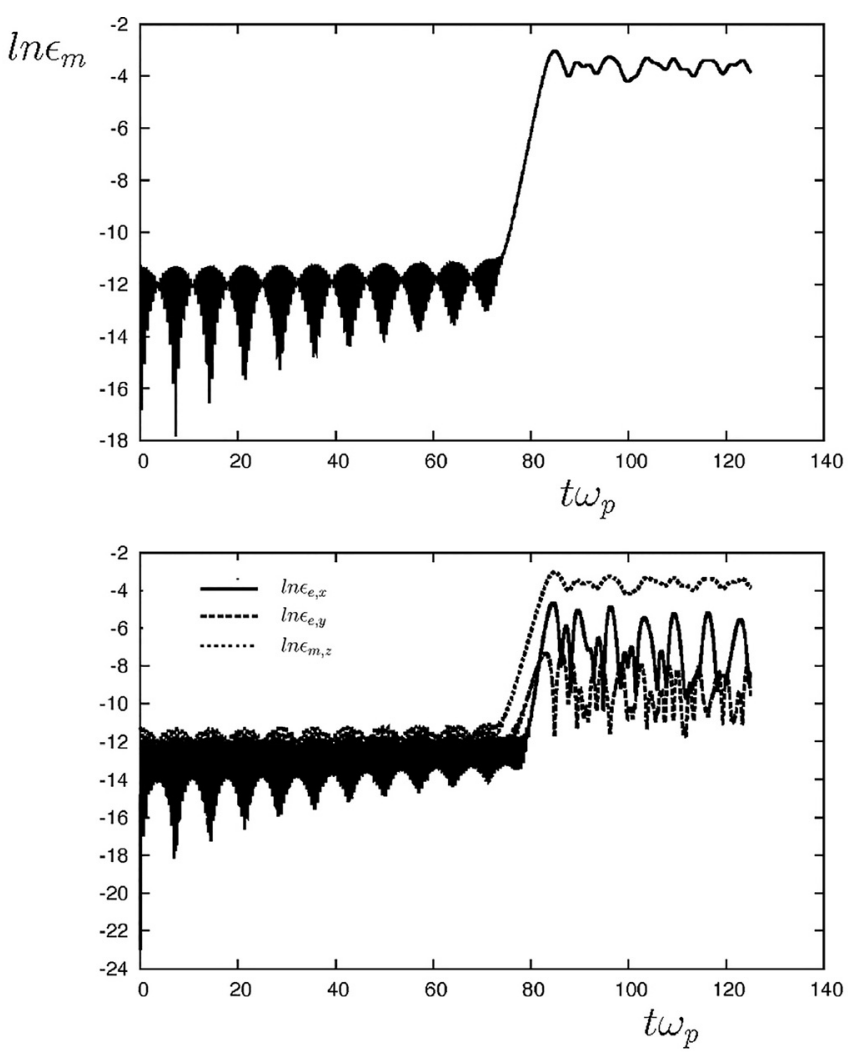

FIG. 16. Time evolution, on a logarithmic scale, of the total magnetic energy (top panel) and the magnetic and electric field energy contributions (bottom panel). The simulation corresponds to the simultaneous excitation of modes $A$ and $B(2 \mathrm{D} 2 \mathrm{~V}$ code).

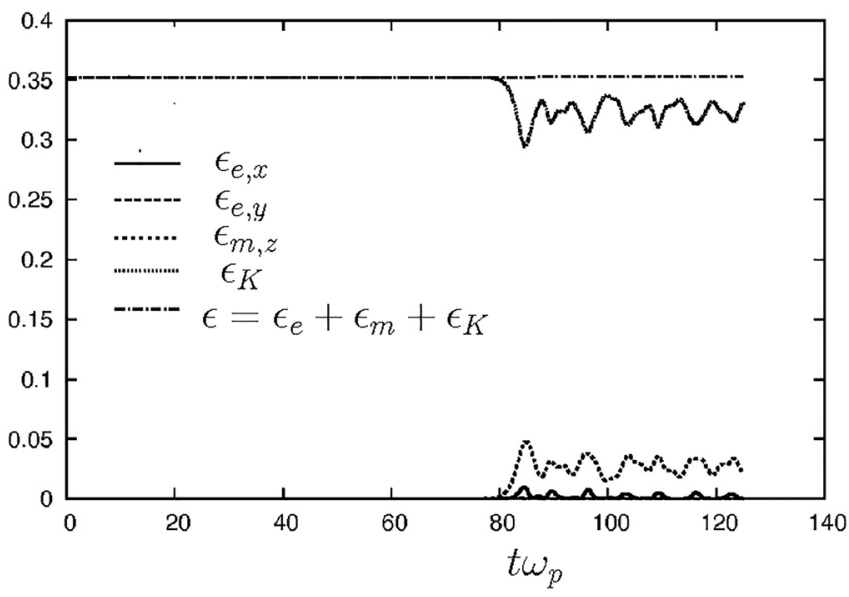

FIG. 17. Time evolution of the kinetic energy $\epsilon_{K}$, the magnetic energy $\epsilon_{m}$, the electric energy of two components $\epsilon_{\theta, x}$ and $\epsilon_{e, y}$, and the total energy $\epsilon=\epsilon_{K}+\epsilon_{m}$ $+\epsilon_{e, x}+\epsilon_{e, y}$. In this regime, the energy transfer is mainly from the kinetic to the magnetic component, with the electric energy contribution being almost negligible. Notice the very good conservation of the total energy. The simulation corresponds to the simultaneous excitation of modes $A$ and $B(2 \mathrm{D} 2 \mathrm{~V}$ code) 

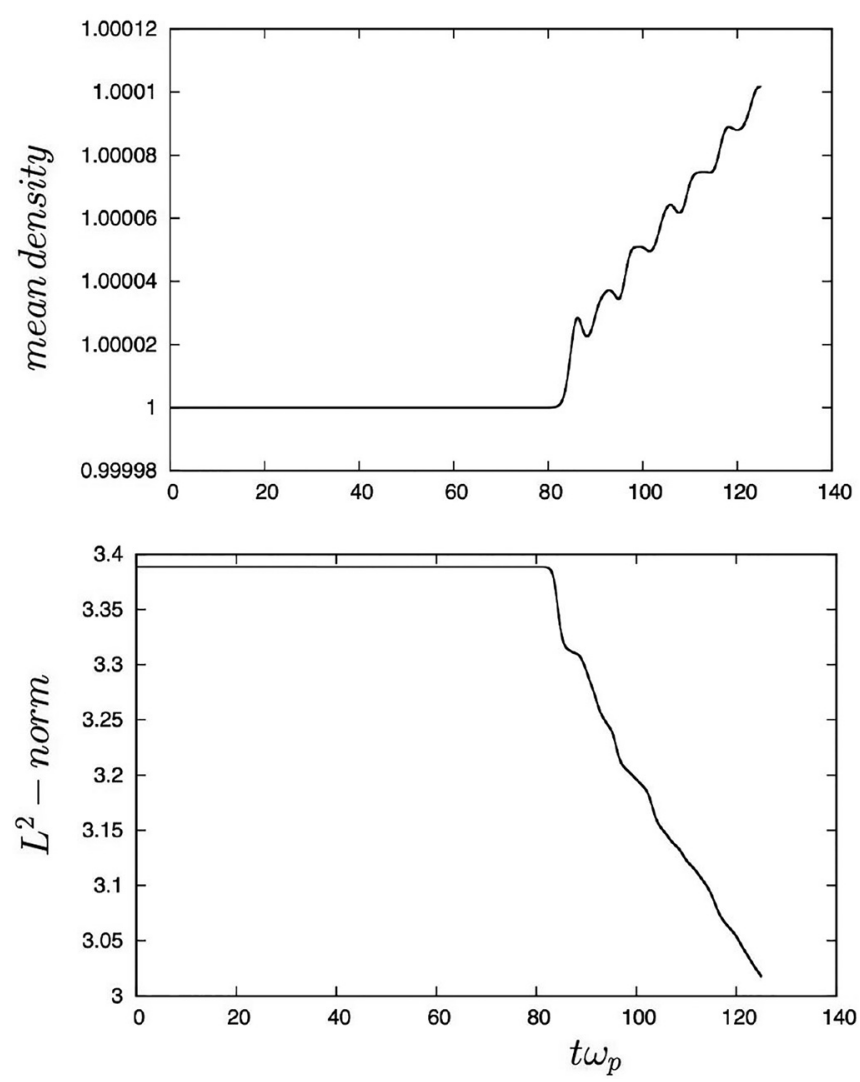

FIG. 18. Time evolution of the total mass (top panel) and the $L^{2}$-norm for the case of simultaneous excitation of modes $A$ and $B$ using the $2 D 2 \mathrm{~V}$ VLEM code. The relative total variations of $10^{-4}$ for the mass and $10 \%$ for the $L^{2}$-norm evidence good conservations granted by the numerical schemes.

$k_{y} d_{e}=5$, and its amplitude increases in time up to a maximum value of $e B_{z \max } / m \omega_{p} \sim 0.3$ (not shown here). This leads to a magnetic bounce frequency,

$$
\frac{\Omega_{b}}{\omega_{p}}=\sqrt{\frac{|\boldsymbol{k}| c}{\omega_{p}} \frac{p_{1}}{m c} \frac{e B_{\max }}{m \omega_{p}}} \simeq \sqrt{5.38 \times 0.9 \times 0.4} \simeq 1.20,
$$

which is found to be in good agreement with the previous result of case B given by Eq. (29).

A low frequency oblique component is visible at the beginning of saturation at time $t \omega_{p}=81$. In this stage of the oblique instability, a dominant contribution of CFI-type modes can be recognized since the contour plots of the $E_{x}$ and $E_{y}$ electric field component (lower panels) are weakly inhomogeneous along $y$, direction of the counterstreaming beams. Note that the $E_{x}$ component oscillates with an harmonic two in the $x$ direction as a result of nonlinear effects (i.e., it is a second order harmonic).

These results show that magnetic trapping is not the dominant mechanism at play at saturation. The analysis of the electric field components, plotted in Fig. 19 (on bottom panels), shows that $E_{x} \sim E_{y}$ in amplitude. Finally, we can estimate the electric bounce frequency,

$$
\frac{\omega_{b}}{\omega_{p}}=\sqrt{\frac{|\boldsymbol{k}| c}{\omega_{p}} \frac{e|\boldsymbol{E}|}{m \omega_{p} c}} \sim 0.46-0.50,
$$

for a value of the modulus of the wavevector $|\boldsymbol{k}| d_{e}$ taken in the interval $[5.38,6.40]$. This value is in good agreement with the value $\omega \simeq 0.48 \omega_{p}$ measured in the simulation, thus confirming the previous results of Eq. (28).

Finally, the phase-space representation of the electron distribution function in the $x$ - $p_{x}$ plane (Fig. 20) shows an electric trapping saturation scenario similar to that displayed for case B. The kinetic heating, in the form of a broadening of the distribution function in the $p_{x}$ space, is clearly observed as a result of the emergence and growth of coherent trapping structures during the saturation of the oblique instability. Figure 20 evidences the formation of "electron holes" as the result of particles trapped in the electric potential. These are observed at time $t \omega_{p}=90$ and are a clear indication of BernsteinGreene-Kruskal (BGK)-type vortices occurring in the nonlinear regime (see Refs. 29 and 62). Such trapping structures are unstable and finally disappear at later times. Particles are accelerated in the proximity of the phase velocity of such acoustic oblique modes. Its value is $v_{\varphi}=\omega / k \sim 0.48 \omega_{p} / 5.38 d_{e}^{-1}=0.089 c$ close to the thermal velocity $v_{\text {th }} \simeq 0.1 \mathrm{c}$.

Figure 21 shows the dependence of $f$ on $p_{x}-p_{y}$ momentum space at two different times (top panels) and on $y-p_{y}$ at the same times (bottom panels). It is clear that the dynamics of the two beams is strongly modified during the evolution of the instability: in particular, in the nonlinear stage, the second beam (of negative velocity) has a lower density, which indicates a transfer of momentum as well.

\section{REVERSIBLE ENERGY TRANSFER: MAGNETIC ENERGY AMPLIFICATION AND COLLISIONLESS HEATING PROCESS}

We now show how the nonlinear mode coupling, occurring when a large spectrum of low frequency (B-type) unstable modes is excited, can lead to a new kinetic heating scenario in the plasma. This occurs as the result of a two-step process.

Step I: First, the excitation of a wide spectrum of magnetic perturbations of kind (26) given on wave-numbers corresponding to both $A$ type and B-type OI solutions (see Fig. 2) leads to the linear growth of oblique modes coupled with CFI modes (Secs. V A and V B). These instabilities lead to a conversion of the kinetic energy of the initial counterpropagating beams into magnetic energy. When a few oblique modes are involved, just a small amount of the kinetic energy is transferred to an electrostatic energy contribution. This $\epsilon_{K} \rightarrow \epsilon_{m}$ process has been detailed in Secs. V A (case A) and V B (case B) and is typical of Weibel-type instabilities. The nonlinear stage sees a dominance of wavelengths $k d_{e} \gtrsim 1$, related to the small wave-number oblique modes, and both magnetic and electric trapping processes take place in the saturation of these instabilities.

Step II: Second, after saturation of the coupled OI/CFI, a wide spectrum of type-B large wave-number oblique modes emerges. In this case, saturation still occurs with $1 \leqq k d_{e} \lesssim 10$ because of the combined action of magnetic trapping and electrostatic trapping. Here, however, because of the presence of a wide nonlinear spectrum of initially unstable modes, the nonlinear coupling leads to the emergence B-type modes with higher wave vectors than before. This regime is characterized by a wavelength spectrum with $k d_{e} \gg 1$ (for practical 

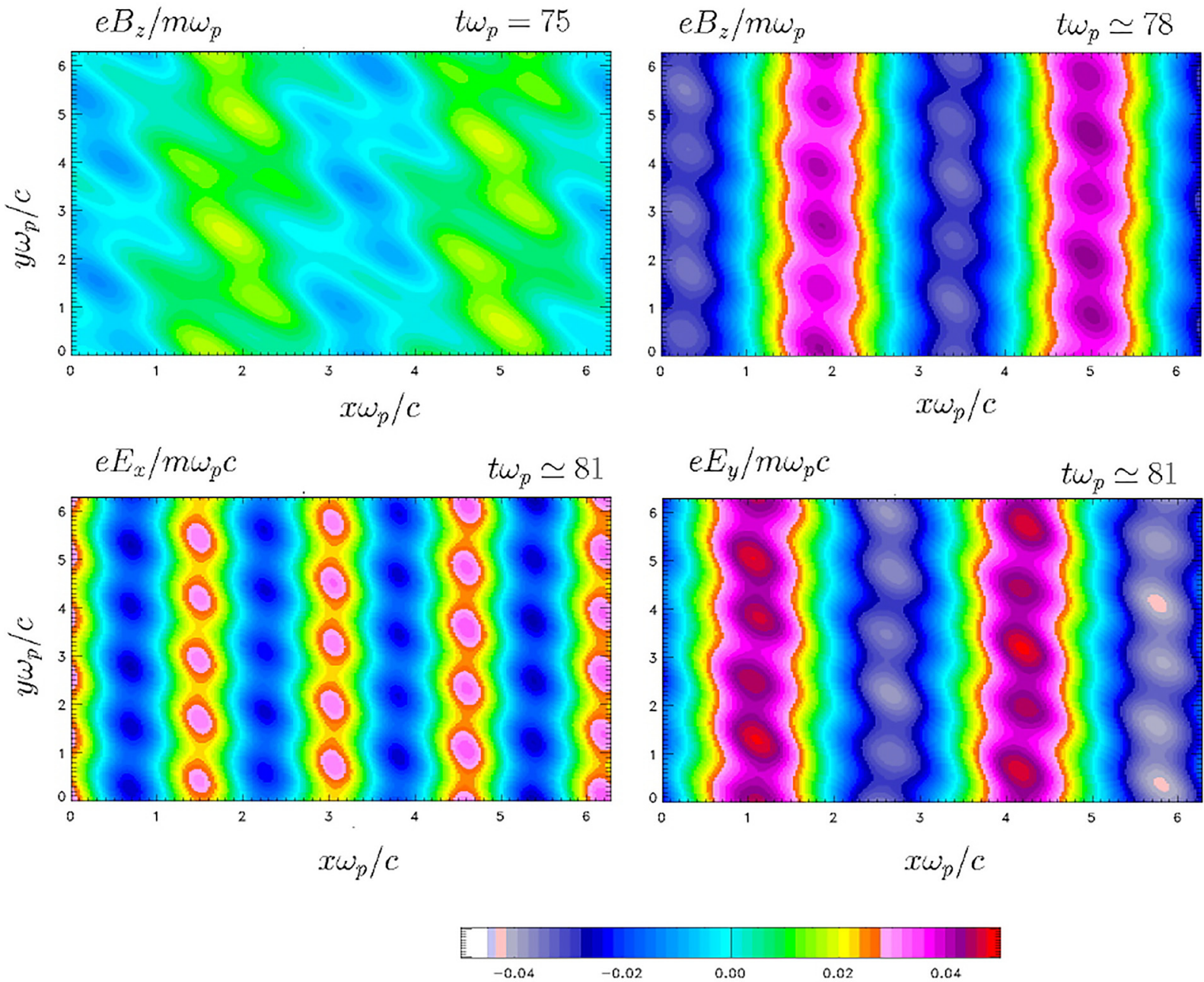

FIG. 19. Plots of the three (normalized) components of the electromagnetic field: the two upper frames show the evolution of the $B_{z}$ component of the magnetic field at two different times. Bottom frames show the plots of the $E_{x}$ (bottom left) and $E_{y}$ (bottom right) components of the electric field. Both magnetic and electrostatic trapping processes, at play at saturation, are evident. The simulation corresponds to simultaneous excitation of modes $A$ and $B$ (2D2V code).

purposes and based on numerical results, we could quantify it as $\left.k d_{e} \gtrsim 10\right)$, and the corresponding saturation mechanism differs from before (cases A, B, and A+B). A process similar to a direct cascade met in turbulence arises, and a transition from low- $k$ B-type oblique modes to large- $k$ B-type oblique modes takes place. Such a transition is indeed accompanied by an amplification of the level of microscopic fluctuations of the distribution function in the coordinate space first (these are related to density variations associated with the electrostatic fluctuations) and then in the velocity space because of the subsequent electron acceleration. Such strong fluctuations can also give rise to second-order effects that lead to a variation of the entropy of the system, which is unrelated to numerical coarse graining (see Secs. II A and II B). The initial energy transfer $\epsilon_{K} \rightarrow \epsilon_{m}$ that is usually met in Weibel-type instabilities [cf. Step (I) above] is now reversed: magnetic energy built up during the linear growth of the OI is transferred into highly fluctuating electric fields linked to the emergence of B-type high-wavenumber oblique modes, and this leads to a stochastic plasma heating mechanism. That is, an energy conversion $\epsilon_{m} \rightarrow \epsilon_{e} \rightarrow \epsilon_{K}$ is here measured.

In order to elucidate the points above, let us discuss the results of a numerical simulation performed with the $2 \mathrm{D} 3 \mathrm{~V}$ version of the code. The initialization is similar to that of case $B$ of Sec. VB but for the much wider spectrum of wave-numbers, which are initially excited: this time, ten modes are introduced in both $k_{x}$ and $k_{y}$ wave numbers with random phases $\varphi_{n_{x}, n_{y}}$ into a perturbation on $B_{z}$ of the form of (24),

$$
B_{z}(x, y, t=0)=B_{0} \sum_{n_{x}=0}^{9} \sum_{n_{y}=0}^{9} \sin \left(n_{x} \Delta k_{x} x+n_{y} \Delta k_{y} y+2 \pi \varphi_{n_{x}, n_{y}}\right) .
$$

The initial amplitude of the perturbation is $e B_{0} / m \omega_{p}=0.05$. Note that both small (i.e., type B) and large wavenumber (i.e., type A) modes are initially excited this way. 

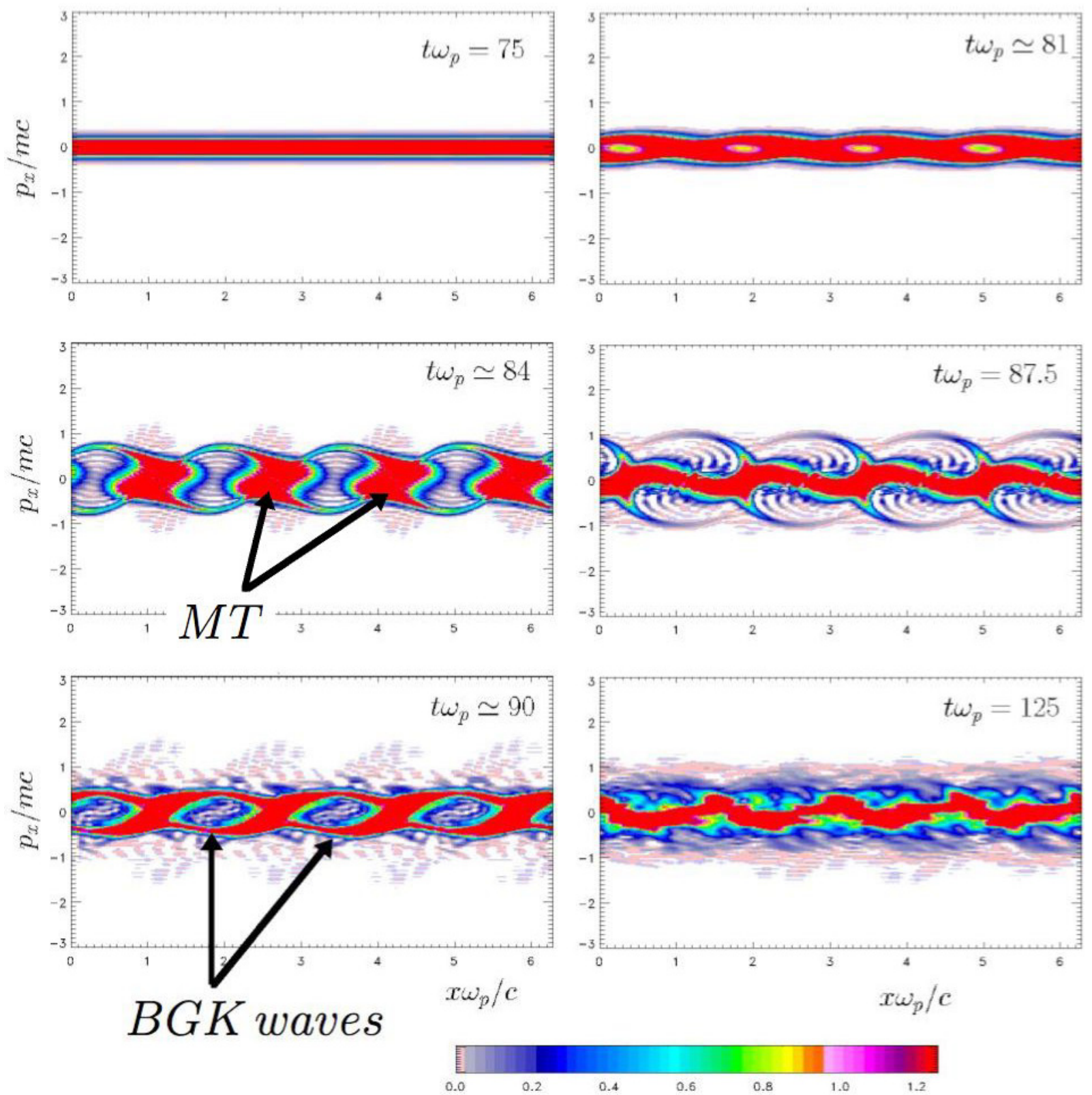

FIG. 20. Representation of the distribution function in the $x-p_{x}$ phase space at six different times during the plasma evolution. Both magnetic trapping structures (evidenced as "MT" in the middle left frame) and electrostatic trapping structures (in the form of BGK waves, evidenced in the bottom left frame) are present. The simulation corresponds to simultaneous excitation of modes $A$ and $B(2 \mathrm{D} 2 \mathrm{~V}$ code)

The other parameters of the simulation are $u_{1}^{(0)}=-u_{2}^{(0)}=0.9$, $L_{x} \simeq 4.0879 d_{e}$, and $L_{y} \simeq 2.5132 d_{e}$, and the extension of the domain in the momentum coordinate corresponds to $\left|p_{i}\right| \leq 3 m c$ for $i=x, y, z$. This phase-space domain has been sampled with $N_{x} N_{y}=128^{2}$ and $N_{p_{x}} N_{p_{y}} N_{p_{z}}=32^{3}$ grid points. This simulation has required 20000 iterations and a time step $\Delta t \omega_{p}=0.0075$. We have also checked the validity of the results we are going to discuss by increasing the sampling in the coordinate space (up to $N_{x} N_{y}=256^{2}$ ): the global scenario presented below has been confirmed.
The results of this simulation are discussed in Figs. 22-28.

In Fig. 22, we have plotted the time evolution of the kinetic energy $\epsilon_{K}$ (top panel) and the magnetic energy $\epsilon_{m}$ (bottom panel), which are reproduced on a semi-logarithmic scale in Fig. 23.

The three previous stages of the evolution discussed in the examples of Sec. V here are recognized, although with some remarkable differences, together with a new fourth stage, corresponding to a kinetic heating process: 

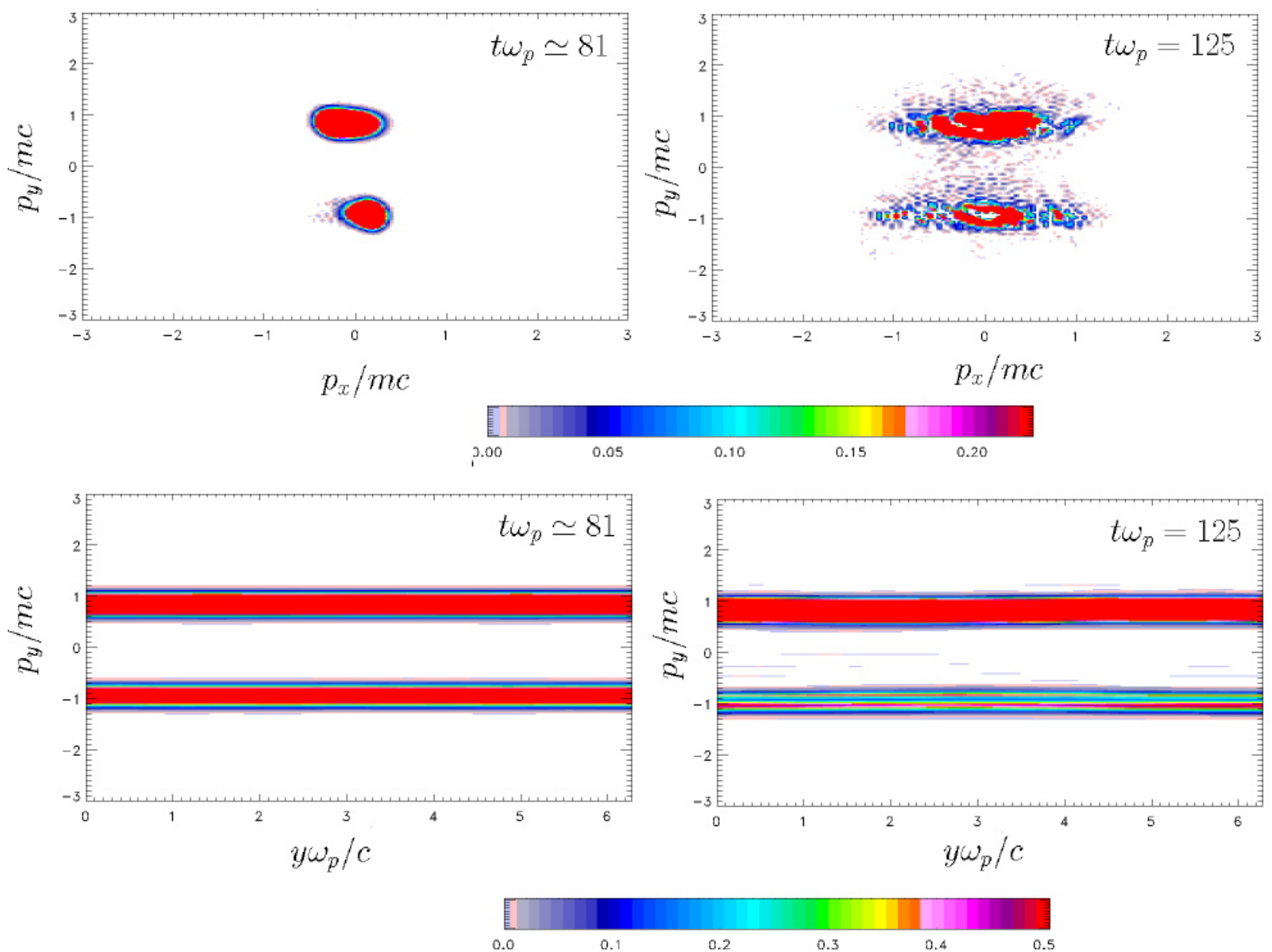

FIG. 21. Representation of $f$ at two different times in the $p_{x}-p_{y}$ momentum space (top panels) and in the $y-p_{y}$ phase space (bottom panels). The dynamics of the beams is strongly modified in the nonlinear saturation regime of the oblique instability, indicating a possible momentum transfer during the interaction. The simulation corresponds to the simultaneous excitation of modes $A$ and $B(2 \mathrm{D} 2 \mathrm{~V}$ code).

(I)

The first stage of the evolution, corresponding to the transient stage occurring for $t \omega_{p} \lesssim 80$, is essentially identical to previous cases in which a narrower spectrum of modes had been excited. The main difference is in the larger number of "spurious" modes that have been excited since the beginning of the simulation due to the wider spectrum. This can be noticed by looking, for example, at the transient time interval in the semi-logarithmic plots of Fig. 23.

(II) The linear stage is well identified, as in previous simulations, in the time interval $80 \leqq t \omega_{p} \lesssim 90$. Here, the amplification of the magnetic energy typical of CFI and B-type oblique modes is, however, accompanied by a more evident increase of the electrostatic energy than before, which is due to the larger contribution from several B-type unstable modes initially excited. At the end of this linear stage $\left(t \omega_{p} \sim 90\right)$, we are in a saturation regime with $1 \lesssim k d_{e}$ $\lesssim 30$, where electrostatic trapping dominates.
(III) The first nonlinear stage can be seen in the time interval $90 \leqq t \omega_{p} \lesssim 105$. This is dominated by the electrostatic particle trapping, which leads to a metastable equilibrium.

(IV) At the end of this nonlinear stage, at $t \omega_{p} \gtrsim 105$, the beamplasma system enters in a regime where $k d_{e} \sim 30$ is the dominant wavelength; more precisely, we have $k_{x} d_{e}$ $\sim 20 \Delta k_{x} d_{e} \sim 30.74$ along $x$ and $k_{y} d_{e} \sim 4 \Delta k_{y} d_{e} \sim 10$ along $y$. The reversal of the energy transfer mechanism characterizes then the whole, subsequent nonlinear dynamics: the magnetic energy is now transferred to the electric field and then to plasma particles, which are accelerated and heated.

As a further cross-check of the accuracy of the numerical results, for this simulation, we have provided in Fig. 24 the time evolution of the components of the total axial momentum $\tilde{C}$ introduced in Sec. II B [Eq. (14)]. In the double periodic spatial geometry of the VLEM solver, both the $x$ and $y$ components of $\tilde{\boldsymbol{C}}$ are expected to be conserved but 

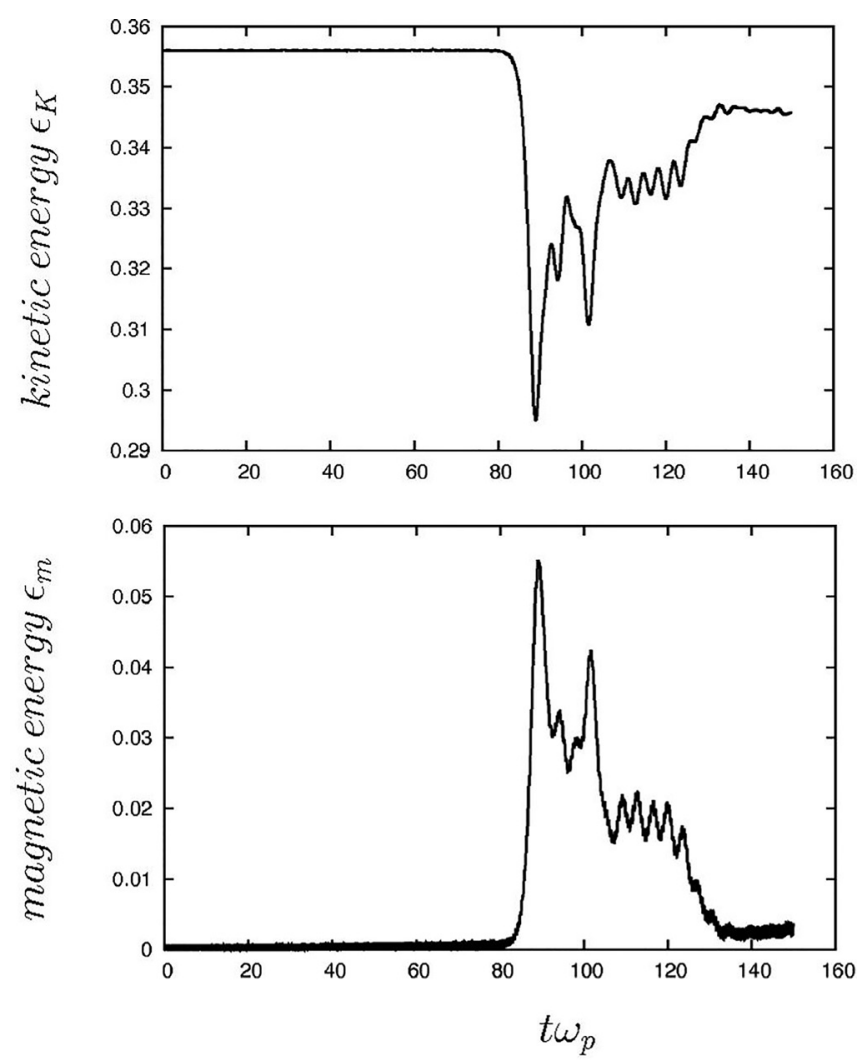

FIG. 22. Plots of the time evolution of the kinetic energy $\epsilon_{K}$ (top panel) and the magnetic energy $\epsilon_{m}$ (bottom panel) for the simulation case $B$ in which a broad spectrum of low frequency modes has been excited. The first stage of the instability in the low-frequency regime is recovered with the exponential growth of a strong magnetic field. At time $t \omega_{p} \sim 100$, the energy transfer is reversed and the magnetic energy is transferred to the plasma particles, therefore inducing a heating process (2D3V code).

for possible "second order effects" related to large amplitude fluctuations of $f$, as discussed in Sec. II B. This is shown in the upper $\left(\tilde{C}_{x}\right)$ and middle panels $\left(\tilde{C}_{y}\right)$ of Fig. 24, where oscillatory fluctuations of the two quantities appear after $t \omega_{p} \gtrless 90$ [stages (III) and (IV) above]. The absolute variation of $\tilde{C}_{x}$ is about 0.02 in amplitude around its mean zero value, whereas the axial component $\tilde{C}_{y}$, although slightly decreasing on average (probably due to dissipation), is even better preserved during the whole simulation, as it maintains a global absolute variation of amplitude 0.01 or less around its initial zero value. These fluctuations appear to be compatible with those of the entropy, which occur at the same times and which we are going to discuss at the end of this section. In the bottom panel of Fig. 24, we then show the time evolution of the $z$ component of $\tilde{\boldsymbol{C}}$, which, in this geometry, is $\tilde{C}_{z}=C_{z}$, since the $z$-component of the Poynting vector is zero in this problem. In this case, $C_{z}$ coincides with the canonical transverse momentum $P_{c z}=p_{z}+e A_{z}(x, y, t)=p_{z}$ since the component $A_{z}$ of the potential vector is here null. Therefore, the conservation of $\tilde{C}_{z}=C_{z}=p_{z}$ is here granted by the fact that $z$ is a cyclic variable (i.e., because of the $\partial / \partial_{z}=0$ geometry restriction). Despite the linear growth due to the cumulation of numerical errors at each step of the iteration, the
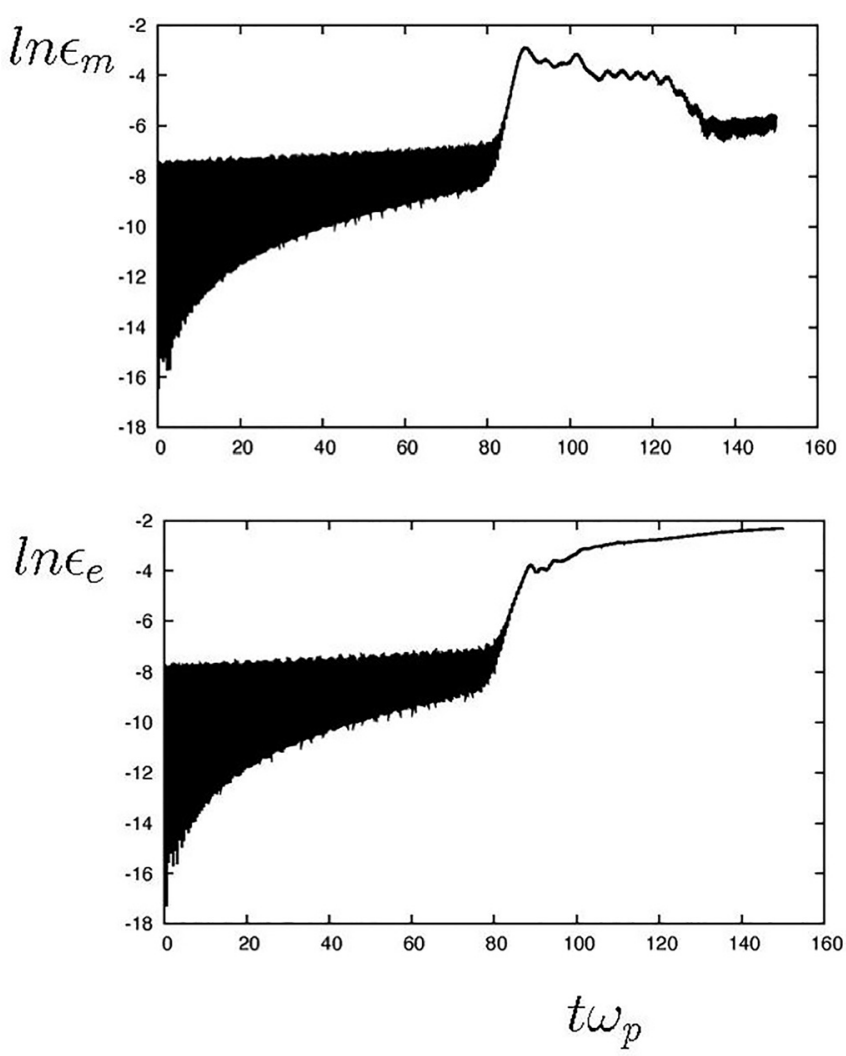

FIG. 23. The temporal evolution of the magnetic (top panel) and electric (bottom panel) energies already shown in Fig. 22 is here reproduced on a logarithmic scale (2D3V code).

conservation shown of $\tilde{C}_{z}$ during the whole simulation is excellent, with its variation being comparable to the rounding errors of the computer in the double precision calculations that we have adopted for these simulations. Also, note that the conservation of the canonical momentum for this kind of problem had been already used in the "multi-stream" approach of Refs. 26, 29, 30, and 84, and its correctness had been already verified in a number of works (see Ref. 67 and references therein for a direct comparison with full-Vlasov simulations).

In order to provide a more detailed view of the physical mechanisms involved, we have also represented the contour-plots of the three components of the electromagnetic field at three different times during the simulation: the $B_{z}$ field (in Fig. 25), the $E_{x}$ field (in Fig. 26), and the $E_{y}$ field (in Fig. 27). In Fig. 25, we can follow the growth of the oblique mode $\left(n_{x}, n_{y}\right)=(1,2)$, until the emergence at time $t \omega_{p}=$ 90 of a structure in the profile of $B_{z}$, which is related to the filamentation instability (i.e., with $\left.\left(n_{x}, n_{y}\right)=(1,0)\right)$ but is characterized by the presence of vortices related to electrostatic trapping (the transient behavior is so short that magnetic trapping does not have enough time to occur). Later, at time $t \omega_{p}=150$, a structure corresponding to a $\left(n_{x}, n_{y}\right)=(3,3)$ spatial modulation becomes visible.

Inspection of the surface plots of the electric field components shows that the transition between low- $k$ and large- $k$ B-type modes (i.e., the emergence of low frequency modes with smaller wavelengths) is accompanied by the generation of thinner and thinner 

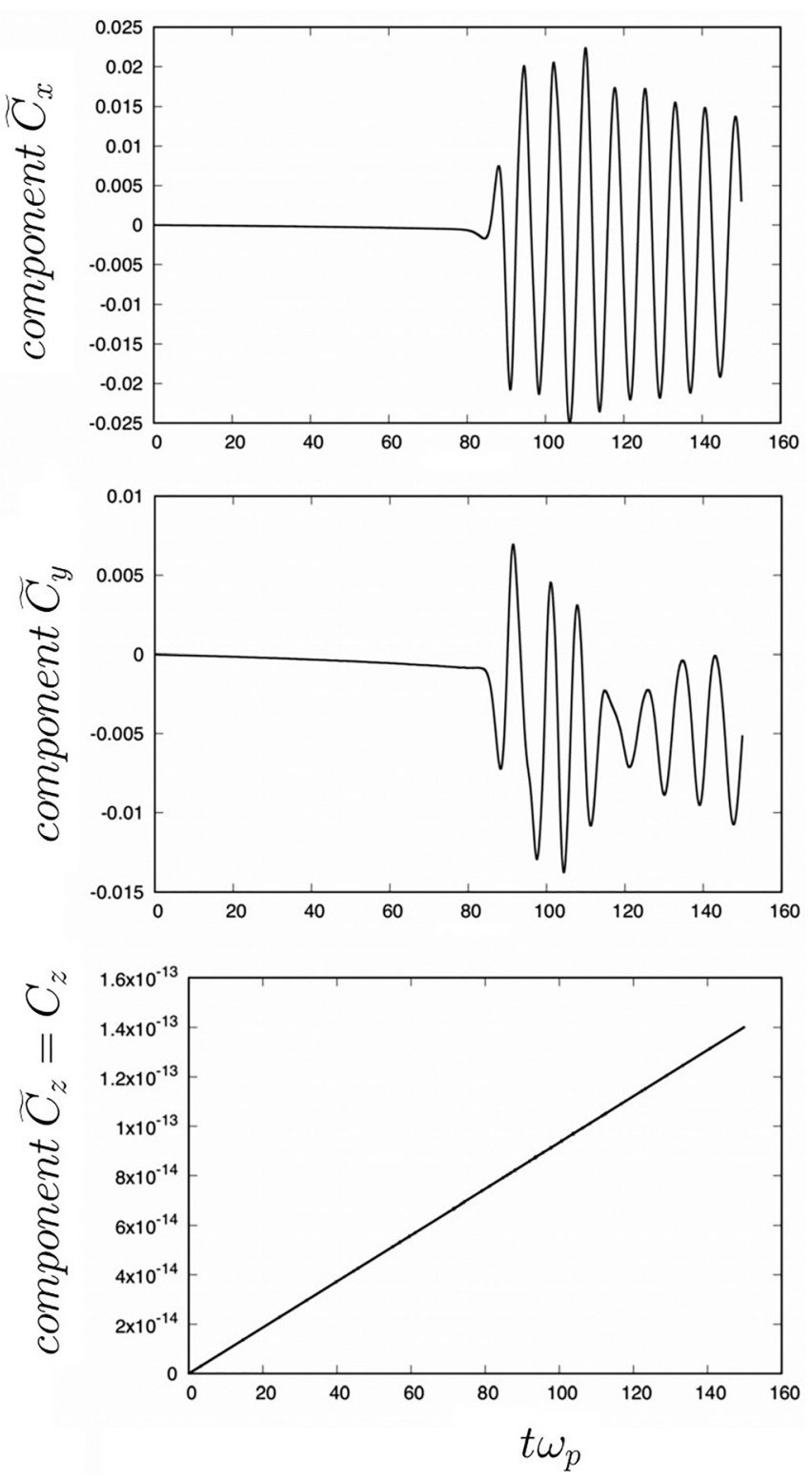

FIG. 24. Conservation of the components of the total momentum $\tilde{\boldsymbol{C}}$. The $\tilde{C}_{x}$ component is in the top panel, the axial component $\tilde{C}_{y}$ is in the middle panel, and the $\tilde{C}_{z}$ component, here equivalent to the $z$ component of the conserved canonical momentum, is in the bottom panel ( $2 \mathrm{D} 3 \mathrm{~V}$ code).

filaments in the coordinate space. At $t \omega_{p}=150$ these small-scale structures appear quite clearly to be superimposed to a dominant $\left(n_{x}, n_{y}\right)=(1,0)$ mode in the profile of $E_{x}$. Figures 26 and 27 also show that at least two highly oscillating oblique B-type modes with different orientations are present: this can be recognized in the time variation of the orientation of the filaments with respect to the $(x, y)$ axes. These high-wave number spatial oscillations of the components of the electric field, which have not been observed in previous simulations of the oblique instability, can lead to "second-order effects" related to the fluctuations of the distribution function. The latter have been already noted by Taggart et al. in PIC simulations of the Weibel instability. ${ }^{63}$

Some details of the kinetic heating mechanism described above in relation to the repartition of the energy among its components are visible in Fig. 28. Here, in the top frame, the dependence of the distribution function on the $x-p_{x}$ phase space coordinates at two different times, corresponding to just before the linear growth of the oblique mode instability and to the end of the simulation, is shown. The latter time is in the advanced nonlinear stage of the instability. This is dominated by the generation of small spatial filaments in the electric field components and by the stochastic saturation mechanism: the evident "heating" that corresponds to the broadening of the distribution along the $p_{x}$ coordinate is a stochastic process that is no longer associated with the presence of coherent trapping structures in the phase space (cf. for comparison Fig. 20). In the middle frame, the time variation of the volume integral of the $x x$ component of the material stress tensor of Eq. (10), i.e., of $\int \tilde{\Pi}_{x x}\left(d^{3} x / V\right)$, is shown. Since this quantity contains both the kinetic energy associated with the motion along $x$ and the contribution from the relativistic internal energy, its increase in time during the evolution of the instability clearly evidences the progressive heating of the electrons during the different stages of the instability. In the linear phase $\left(80 \leqq t \omega_{p} \lesssim 90\right)$, this occurs because of the acceleration of the electron in the $x$ direction, orthogonal to that of the initial beams: on the one hand, this agrees with the identification of the counterpropagating cold beam configuration with an initial temperature $T_{y}$ in the $y$-momentum component and a null temperature $T_{x}$, which thus corresponds to an initial temperature anisotropy like it happens ${ }^{68}$ for pure Weibel instability. ${ }^{24,25}$ On the other hand, and thanks to such an identification, it agrees with the thermodynamical interpretation of Weibel-type anisotropydriven instabilities, in which the electron plasma acts as a thermodynamical machine that generates work in the form of a magnetic field by operating in between two thermal sources (the electron temperatures parallel and perpendicular to $\boldsymbol{k}$ ), thus tending to the thermal isotropization of the two temperature components (see Refs. 69 and 70 for a description based on the fluid-like quantities). Notice that we can also recognize the four different stages of the dynamics of the OI in the plot of $\int \tilde{\Pi}_{x x}\left(d^{3} x / V\right)$ vs time (middle frame). The two first stages (I) and (II) are clearly visible. At saturation, starting from the transient equilibrium period (III), we observe in the time interval $90 \lesssim t \omega_{p} \lesssim 120$ the beginning of an oscillatory behavior induced by the electrostatic trapping mechanism at the frequency $\omega \simeq 0.49 \omega_{p}$. This numerical value is in good agreement with the theoretical values of Eqs. (28) and (32). Finally, these oscillations suddenly stop at time $t \omega_{p} \sim 120-140$, during the advanced nonlinear stage (IV) characterized by the $\varepsilon_{m} \rightarrow \varepsilon_{k}$ energy transfer: this corresponds to the time in which the electrostatic-induced kinetic heating takes place.

Finally, in the bottom frame in Fig. 28, we show the time evolution of Shannon's entropy (6) during the whole simulation. The plot of $S$ vs time displays the expected behavior characteristic of the three first stages of the instability, as well as a new behavior that can be related to stage IV: we first note the initial equilibrium (I) for $0 \leq t \omega_{p} \leq 80$, the linear stage (II) for $80 \leq t \omega_{p} \leq 90$ associated with the growth of the entropy, and then the metastable equilibrium (III) at 

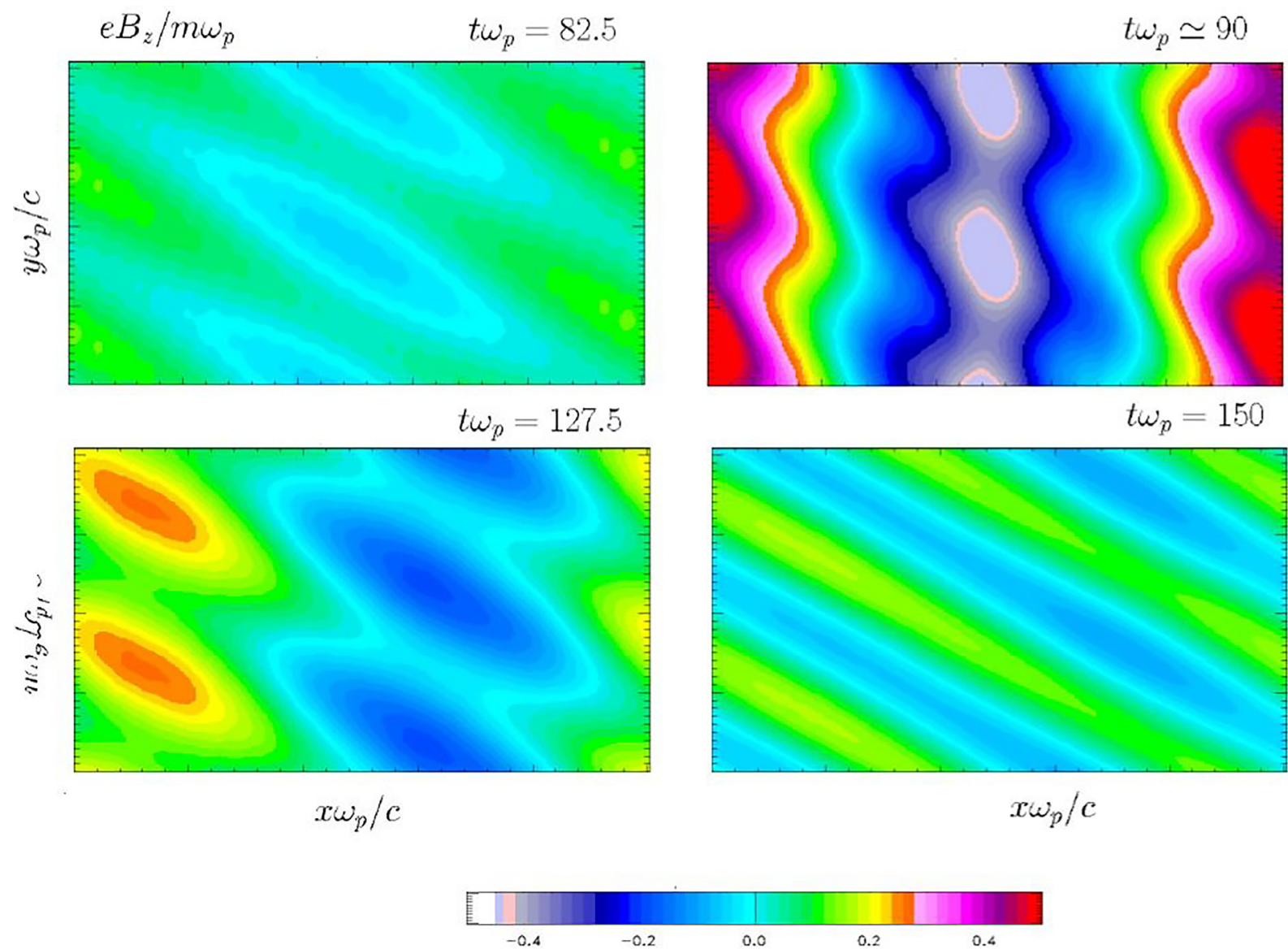

FIG. 25. Contour-plots of the normalized $B_{z}$ field components at four instants, for the simulation case in which a broad spectrum of low-frequency $B$-type modes has been excited. The growth of a dominant oblique mode $\left(n_{x}, n_{y}\right)=(1,2)$ is observed until the emergence of trapping structures at time $t \omega_{p}=90$ and then of an oblique spatial modulation (at almost $45^{\circ}$ with respect to the Cartesian axes) at time $t \omega_{p}=150$ (2D3V code).

saturation for $90 \leq t \omega_{p} \leq 105$. Then, a surprising behavior is observed: for $t \omega_{p} \gtrsim 105, S$ decreases.

The increase in $S$, observed during the stage (II), is due to a loss of macroscopic information of the phase-space dynamics: it is exponential as long as the linear oblique instability exponentially fosters the phase-space filamentation process. Then, in stage III, a metastable saturation is reached (this time earlier than in previous simulation cases) between $t \omega_{p} \simeq 90$ and $t \omega_{p} \simeq 105$, in which the value of $S$ remains, but for fluctuations, more or less constant. Here, the electrostatic trapping-type saturation has led to stop the numerical entropy production because of a "physical" smoothing of the phase-space structures. However, as the nonlinear generation of fine structures in the coordinate space goes on because of the cascade toward high wave numbers, which is related to the nonlinear coupling of the electrostatic component of type-B oblique modes, large amplitude fluctuations of the distribution function appear. This, in agreement with the discussion developed in Sec. II B, violates the strict condition that grants the mathematical conservation of $S$, which can, therefore, either increase or decrease "because of physical effects" related to these second order fluctuations of $f$. In particular, the emergence of organized structures in the coordinate space (the filamentary structures in the contourplots of the electromagnetic field components of Figs. 25-27) results to be compatible with an overall entropy decrease, which is observed for $t \omega_{p} \gtrsim 105$. This a posteriori suggests that the entropy increase during stages (I) and (II) of the evolution has also occurred in an almost reversible way, again due to large amplitude fluctuations of $f$ related to the current filamentation. This point, which deserves further investigation, would mean that just a little amount of microscopic information is irreversibly lost because of the combined effects of filamentation and coarse graining during the growth of the oblique unstable modes. This results in a little, global entropy increase from the initial to the final state: the large part of macroscopic information lost by phase-mixing in the first stages of the evolution results to be later recovered [stages (III) and (IV)], similar to what happens for plasma echoes.

\section{CONCLUSION}

Numerical experiments have been performed to investigate the nonlinear dynamics of two classes of modes, recently identified, ${ }^{1}$ of the oblique instability. A particular focus has been put on the investigation of a mechanism of transition between two classes of modes that 


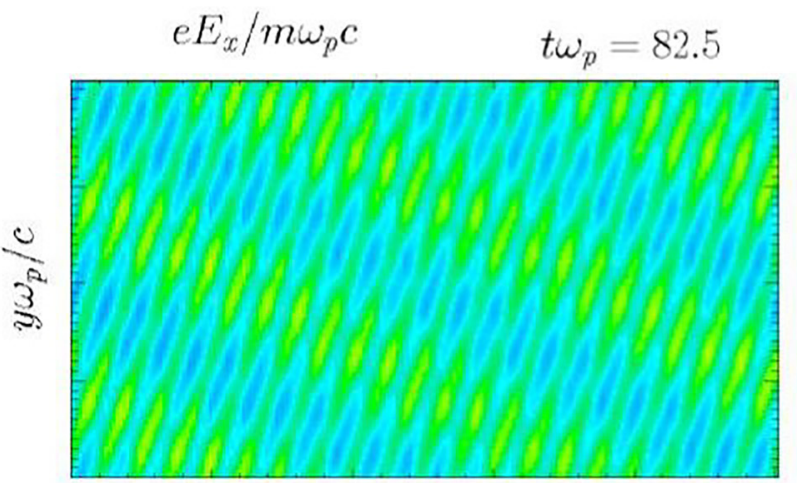

thin filaments

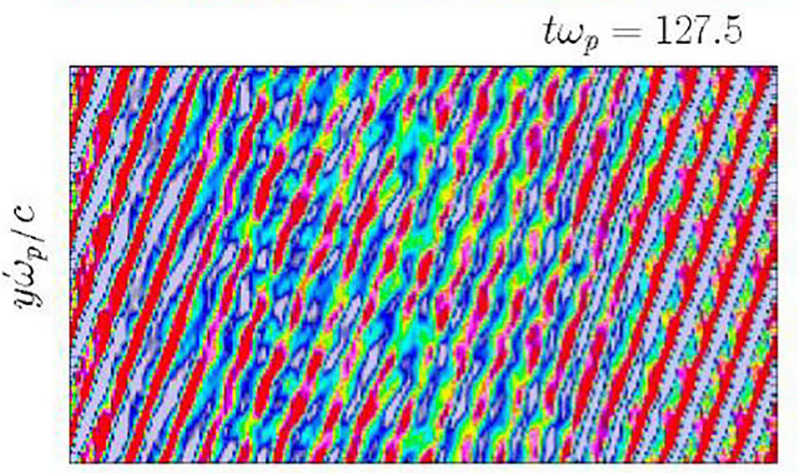

$x \omega_{p} / c$

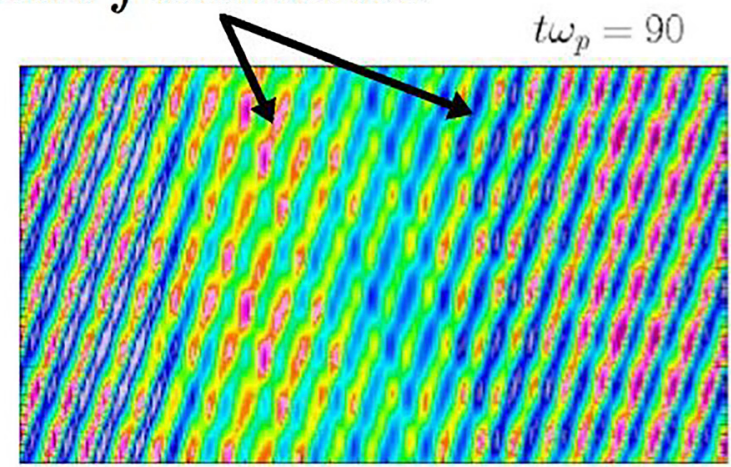

$t \omega_{p}=150$

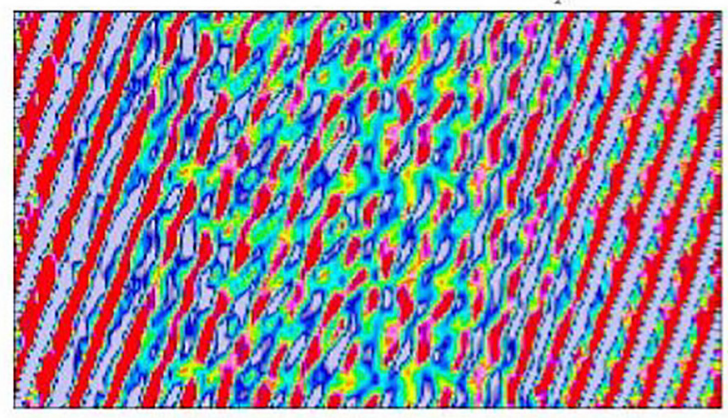

$$
x \omega_{p} / c
$$

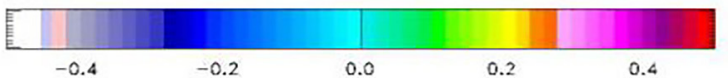

FIG. 26. Contour-plots of the normalized $E_{x}$ field component at the same times as of Fig. 25 (simulation case of excitation of a broad spectrum of $B$-type modes). Note the generation of thin filaments in the configuration space, associated with electrostatic spatial oscillations (2D3V code).

are time-resonant and can co-exist with different values of the real frequency for the same values of the wave numbers. A new plasma heating mechanism driven by this transition has been thus identified and discussed. This mechanism appears to characterize the saturation of the oblique instability for large wave-numbers $\left(k d_{e} \gg 1\right)$, and when a large spectrum of unstable modes is destabilized, it differs from the usual magnetic trapping saturation mechanism previously identified to characterize single Weibel-type modes with $k d_{e} \gtrsim 1$. In particular, it reverses the $\epsilon_{K} \rightarrow \epsilon_{m}$ energy transfer that identifies Weibel-type instabilities because of the highly oscillatory (in space) character of the electrostatic component of these modes. This induces in the nonlinear stage of these electromagnetic instabilities an energy transfer from the magnetic to the electrostatic low-frequency component and then to the electron kinetic and internal energy: $\epsilon_{m} \rightarrow \epsilon_{e} \rightarrow \epsilon_{K}$.

Moreover, the transition of the oblique instability to high wave number modes (of low frequency, i.e., of "B-type," cf. Fig. 2) leads to the formation of a direct (turbulent-like) cascade-type filamentation process of the distribution function in the space coordinate. This process can compete with the filamentation mechanism of the distribution function in the velocity space, an intrinsic mathematical property of the Vlasov equation associated with its weak-convergence properties. We have then provided an example of how an electromagnetic instability (the oblique mode instability) can perturb the natural mechanism of filamentation of the distribution function, which, in a Vlasov plasma, unavoidably leads to the production of thinner and thinner filaments in phase space and which is numerically bound to lead to an irreversible entropy increase. In particular, the large amplitude fluctuations of the distribution function, which are associated with the fine spatial modulation of the perturbed electric field, can violate the conditions that grant the weak convergence of the mathematical solutions of the Vlasov equation, thus allowing a violation of the entropy conservation, which is unrelated to the numerical smoothing of the intrinsic phase-space filamentation of Vlasov plasmas. The spatial organization of the distribution function and the electromagnetic field components (i.e., the fine scale filaments in the coordinate space) is even capable of resulting in induction of an unexpected entropy decrease, which is not a numerical artifact. At the same time, the fact that the system reaches a final value of the entropy that is close to the initial one also indicates that the entropy growth in the first stage of the instability has occurred in an almost reversible way because of a similar mechanism (large 

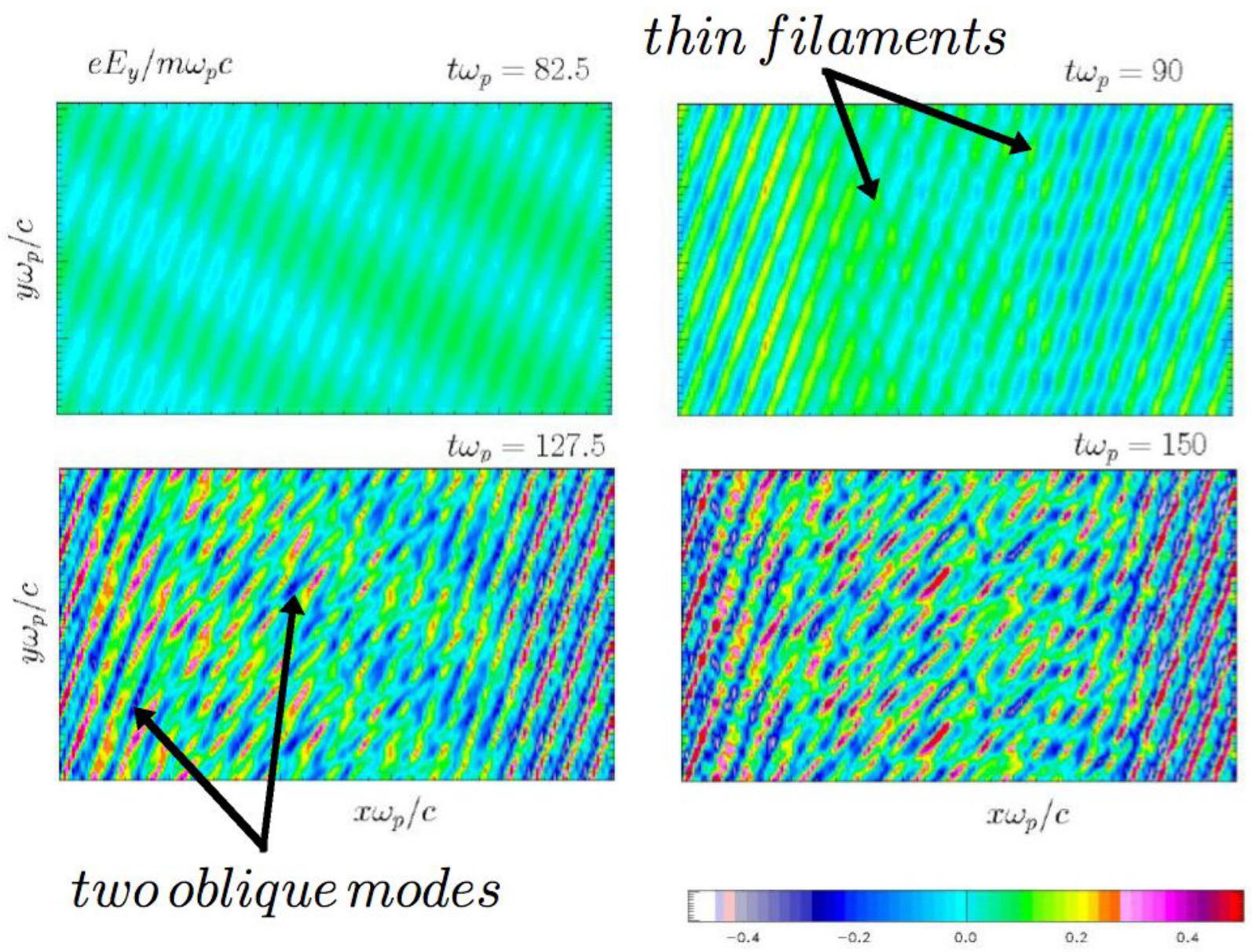

FIG. 27. Plots of the normalized $E_{y}$ field component at the same times of Figs. 25 and 26 (simulation case of excitation of a broad spectrum of $B$-type modes). Notice at time $t \omega_{p}=127.5$ (evidenced by arrows in the bottom left panel) the emergence of two large wavenumber modes whose spatial modulations are associated with different directions in wave vectors $(2 \mathrm{D} 3 \mathrm{~V}$ code).

amplitude fluctuations of $f$ during the exponential growth of the initial oblique modes) and that just a little amount of microscopic information is irreversibly lost because of numerical coarse-graining of phasespace filaments. Two further questions can be then related to this remark: could the "final" metastable state be achieved, in principle, in the absence of dissipation, or is the latter required, however small, in order to provide an "arrow of time," which makes the system converge to such an attractor? In this sense, how much dependent on dissipation is the mostly reversible kinetic heating process that we have evidenced? (could it occur in the absence of dissipation?). Addressing these questions and investigating the implications of this new process deserves, of course, future, dedicated studies.

In addition to this, two further important elements of interest should be included in the analysis we have presented here: on the one hand, the role played by the asymmetry of the beams and, on the other hand, the role played by a possible initial temperature anisotropy of each beam. The latter, of course, opens to the coupling with pure Weibel-type modes, and it is known that the asymmetry of the beams does not only change the solutions of cold CFI and oblique modes ${ }^{31,32}$ but also radically affects the pure Weibel type solutions. ${ }^{70}$ Both these effects thus influence the way that Weibel modes couple to CFI, TSI, and oblique modes. Finally, a more thorough study of the stochastic heating scenario in terms of turbulence cascade will be necessary to assess the implications it can have for the heating of turbulent plasmas in which Weibel-type oblique modes can naturally develop. This is relevant not only to the solar corona and to the solar wind but also to laser-plasma interactions, in which spatial resonances related to the finite size of the laser-accelerated electron beams are known to play a key role in the way that oblique and current-filamentation modes affect the generation of magnetic structures. ${ }^{71}$ A kinetic extension of these fluid results, which keep account of the coupling of spatial and time resonances of Weibel-type modes, is still lacking. It must be, at the same time, noted that recent numerical kinetic studies ${ }^{72,73}$ have pointed out the importance of considering spatial inhomogeneities in the development of Weibel-type modes to account for the persistence of a strong magnetic field capable of generating gamma-ray burst 

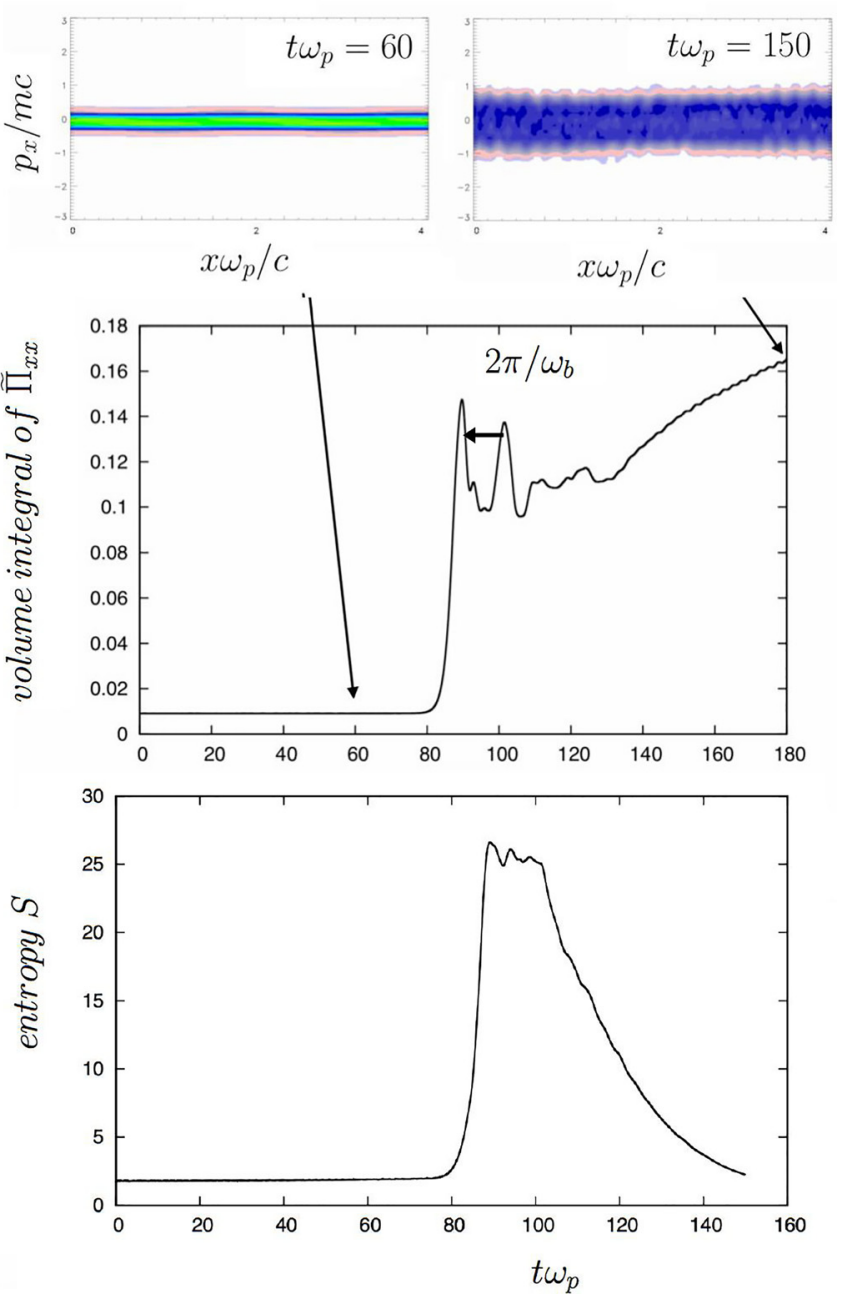

FIG. 28. Some further figures that characterize the kinetic heating scenario in connection to the nonlinear transition to high wavenumber electrostatic modes and to high amplitude fluctuations inducing entropy variations. Top frames: plot of the distribution function in the $x-p_{x}$ phase space at two different times. The broadening in $p_{x}$ over time of the distribution function (top panels) mirrors a heating mechanism of the plasma where the magnetic energy is back-transferred to the thermal energy. Middle frame: time evolution of $\int \tilde{\Pi}_{x x}\left(d^{3} x / V\right)$. The period of the two first peaks evidenced in the figure corresponds to the electrostatic bounce period $2 \pi / \omega_{b}$. Bottom frame: time evolution of the entropy. Note the overall entropy decrease after $t \omega_{p} \simeq 105$, which is compatible with the large amplitude fluctuations associated with the filamentary structures evidenced in the contour-plots of the electromagnetic field components of Figs. 25-27 (2D3V code).

emissions in astrophysical relativistic shocks. We expect the results we have here presented about the regimes of the oblique instability to be relevant to these relativistic, astrophysical environments as well.

\section{ACKNOWLEDGMENTS}

The authors are indebted to the IDRIS computational centre, Orsay, France, for computer time allocation on their computers. This work was granted across to the HPC resources (Grant No.
2019-2020-057290) made by GENCI (Grand Equipement National de Calcul Intensif).

\section{DATA AVAILABILITY}

The data that support the findings of this study are available from the corresponding author upon reasonable request.

\section{REFERENCES}

${ }^{1}$ A. Ghizzo, D. Del Sarto, and M. Sarrat, "Low- and high- frequency nature of oblique filamentation modes in counter-streaming plasmas. I. Linear theory," Phys. Plasmas 27, 072103 (2020).

${ }^{2}$ S. Servidio, F. Valentini, F. Califano, and P. Veltri, Phys. Rev. Lett. 108, 045001 (2012).

${ }^{3}$ F. Valentini, F. Perrone, S. Stabile, and O. Pezzi, New J. Phys. 18, 125001 (2016).

${ }^{4}$ S. Servidio, F. Valentini, D. Perrone, A. Greco, F. Califano, W. H. Matthaeus, and P. Veltri, J. Plasma Phys. 81, 325810107 (2015).

${ }^{5}$ M. Wan, W. H. Matthaeus, H. Karimabadi, V. Roytershteyn, M. Shay, P. Wu, W. Daughton, B. Loring, and S. C. Chapman, Phys. Rev. Lett. 109, 195001 (2012)

${ }^{6}$ Y. Yang, W. H. Matthaeus, T. N. Parashar, C. C. Haggerty, V. Roytershteyn, W. Daughton, M. Wan, Y. Shi, and S. Chen, Phys. Plasmas 24, 072306 (2017).

${ }^{7}$ Y. Yang, W. H. Matthaeus, T. N. Parashar, P. Wu, M. Wan, Y. Shi, S. Chen, V. Roytershteyn, and W. Daughton, Phys. Rev. E 95, 061201(R) (2017).

${ }^{8}$ Y. Yang, M. Wan, W. H. Matthaeus, L. Sorriso-Valvo, T. N. Parashar, Q. Lu, Y. Shi, and S. Chen, Mon. Not. R. Astron. Soc. 482, 4933 (2019).

${ }^{9}$ D. Del Sarto, F. Pegoraro, and F. Califano, Phys. Rev. E 93, 053203 (2016).

${ }^{10}$ D. Del Sarto and F. Pegoraro, Mon. Not. R. Astron. Soc. 475, 181 (2018).

${ }^{11}$ W. H. Matthaeus and S. L. Lamkin, Phys. Fluids 28, 303 (1985).

${ }^{12}$ L. Franci, S. Landi, L. Matteini, A. Verdini, and P. Hellinger, Astrophys. J. 833, 91 (2016).

${ }^{13}$ S. S. Cerri and F. Califano, New J. Phys. 19, 025007 (2017).

${ }^{14}$ J. C. Kasper, A. J. Lazarus, and S. P. Gary, Geophys. Res. Lett. 29, 1839, https:// doi.org/10.1029/2002GL015128 (2002).

${ }^{15}$ P. Hellinger, P. Trávnicek, J. C. Kasper, and A. J. Lazarus, Geophys. Res. Lett. 33, L09101, https://doi.org/10.1029/2006GL025925 (2006).

${ }^{16}$ D. Inscher and R. Schlickeiser, Phys. Plasmas 21, 022110 (2014).

${ }^{17}$ R. Schlickeiser and P. H. Yoon, Phys. Plasmas 21, 072119 (2014).

${ }^{18}$ E. S. Weibel, Phys. Rev. Lett. 2, 83 (1959).

${ }^{19}$ R. A. Fonseca, L. O. Silva, J. W. Tonge, W. B. Mori, and J. M. Dawson, Phys. Plasmas 10, 1979 (2003).

${ }^{20}$ R. Schlickeiser and P. K. Shukla, Astrophys. J. Lett. 599, L57 (2003).

${ }^{21}$ G. Shvets, O. Polomarov, V. Khudik, C. Siemon, and I. Kaganovich, Phys. Plasmas 16, 056303 (2009).

${ }^{22}$ P. H. Yoon, Phys. Plasmas 14, 024504 (2007).

${ }^{23}$ T. Y. B. Yang, Y. Gallant, J. Arons, and A. B. Langdon, Phys. Fluids B 5, 3369 (1993).

${ }^{24}$ B. D. Fried, Phys. Fluids 2, 337 (1959).

${ }^{25}$ A. Inglebert, A. Ghizzo, T. Reveille, D. Del Sarto, P. Bertrand, and F. Califano, Europhys. Lett. 95, 45002 (2011).

${ }^{26}$ A. Ghizzo and P. Bertrand, Phys. Plasmas 20, 082109 (2013).

${ }^{27}$ K. M. Watson, S. A. Bludman, and M. N. Rosenbluth, Phys. Fluids 3, 741 (1960).

${ }^{28}$ S. A. Bludman, K. M. Watson, and M. N. Rosenbluth, Phys. Fluids 3, 747 (1960).

${ }^{29}$ A. Ghizzo, Phys. Plasmas 20, 082110 (2013).

${ }^{30}$ A. Ghizzo, Phys. Plasmas 20, 082111 (2013).

${ }^{31}$ F. Califano, F. Pegoraro, and S. V. Bulanov, Phys. Rev. E 56, 963 (1997).

${ }^{32}$ F. Califano, R. Prandi, F. Pegoraro, and S. V. Bulanov, Phys. Rev. E 58, 7837 (1998).

${ }^{33}$ A. Bret, M. C. Firpo, and C. Deutsch, Phys. Rev. Lett. 94, 115002 (2005).

${ }^{34}$ L. Gremillet, D. Bénisti, E. Lefebvre, and A. Bret, Phys. Plasmas 14, 040704 (2007).

${ }^{35}$ A. Bret, L. Gremillet, D. Bénisti, and E. Lefebvre, Phys. Rev. Lett. 100, 205008 (2008). 
${ }^{36}$ A. Bret, L. Gremillet, and M. E. Dieckmann, Phys. Plasmas 17, 120501 (2010).

${ }^{37}$ A. Bret, L. Gremillet, and D. Bénisti, Phys. Rev. E 81, 036402 (2010).

${ }^{38}$ A. Ghizzo, F. Huot, and P. Bertrand, J. Comput. Phys. 186, 47 (2003).

${ }^{39}$ M. Sarrat, A. Ghizzo, D. Del Sarto, and L. Serrat, Eur. Phys. J. D 71, 271 (2017).

${ }^{40}$ E. Sonnendrücker, J. R. Roche, P. Bertrand, and A. Ghizzo, J. Comput. Phys. 149, 201 (1999).

${ }^{41} \mathrm{R}$. Hakim, Introduction to Relativistic Statistical Mechanics, Classical and Quantum (World Scientific Publishing, New Jersey, 2011).

${ }^{42}$ R. C. Davidson and P. H. Yoon, Phys. Fluids B 1, 195 (1989).

${ }^{43}$ H. Alfvèn, Phys. Rev. 55, 425 (1939).

${ }^{44}$ A. Grassi, M. Grech, F. Amiranoff, F. Pegoraro, A. Macchi, and C. Riconda, Phys. Rev. E 95, 023203 (2017)

${ }^{45}$ R. C. Davidson, D. A. Hammer, I. Haber, and C. E. Wagner, Phys. Fluids 15 317 (1972).

${ }^{46}$ F. Califano, F. Pegoraro, S. V. Bulanov, and A. Mangeney, Phys. Rev. E 57, 7048 (1998).

${ }^{47}$ R. Bermejo, Mon. Weather Rev. 118, 979 (1990).

${ }^{48}$ C. G. Cheng and G. Knorr, J. Comput. Phys. 22, 330 (1976).

${ }^{49}$ P. Bertrand, A. Ghizzo, T. W. Johnston, M. Shoucri, E. Fijalkow, and M. R. Feix, Phys. Fluids B 2, 1028 (1990).

${ }^{50}$ T. W. Johnston, P. Bertrand, A. Ghizzo, M. Shoucri, E. Fijalkow, and M. R. Feix, Phys. Fluids B 4, 2523 (1992).

${ }^{51}$ A. Ghizzo, P. Bertrand, J. Lebas, T. W. Johnston, and M. Shoucri, Phys. Plasmas 3, 650 (1996).

${ }^{52}$ V. Grandgirard, M. Brunetti, P. Bertrand, N. Besse, X. Garbet, P. Ghendrih, E. Sonnendrücker, J. Vaclavik, and L. Villard, J. Comput. Phys. 217, 395 (2006).

${ }^{53}$ A. Staniforth and J. Coté, Mon. Wea. Rev. 119(9), 2206-2223 (1991).

${ }^{54}$ R. Bermejo, Numer. Math. 60, 163 (1991).

${ }^{55} \mathrm{M}$. Falcone and R. Ferreti, SIAM J. Numer. Anal. 35, 909 (1998).

${ }^{56}$ P. Bartello and S. J. Thomas, Mon. Weather Rev. 124, 2883 (1996).

${ }^{57}$ C. Mouhot and C. Villani, J. Math. Phys. 51, 015204 (2010).

${ }^{58}$ C. Villani, Phys. Plasmas 21, 030901 (2014).

${ }^{59}$ R. J. Di Perna and P.-L. Lions, Commun. Pure Appl. Math. 42, 729 (1989).

${ }^{60}$ G. Rein, Commun. Math. Sci. 2, 145 (2004).

${ }^{61}$ A. J. Klimas, A. F. Vinas, and J. A. Araneda, J. Plasma Phys. 83, 905830405 (2017).
${ }^{62}$ I. Bernstein, J. Greene, and M. D. Kruskal, Phys. Rev. 108, 546 (1957).

${ }^{63}$ K. A. Taggart, B. B. Godrey, C. E. Rhoades, and H. C. Ives, Phys. Rev. Lett. 29, 1729 (1972).

${ }^{64}$ M. R. Feix and P. Bertrand, Transp. Theory Stat. Phys. 34, 7 (2005).

${ }^{65} \mathrm{P}$. Bertrand, M. Albrecht-Marc, T. Reveillé, and A. Ghizzo, Transp. Theory Stat. Phys. 34, 103 (2005).

${ }^{66}$ Instead, PIC-type codes would have an initial $(\partial f / \partial t)_{P I C} \propto 1 /\left(n_{q p}(\Delta x)^{d_{x}}\right) \neq 0$ effective term at rhs of (1); cf., Sec. IV A.

${ }^{67}$ A. Ghizzo, M. Sarrat, and D. D. Sarto, J. Plasma Phys. 83, 705830101 (2017).

${ }^{68}$ In reality, since for the Vlasov simulations performed here we have not considered the beams as cold but rather given by Eq. (26), a small isotropic temperature $T$ is present along the $x$ and $y$ momentum directions.

${ }^{69}$ D. S. Lemons and D. Winske, J. Plasma Phys. 23, 283 (1980).

${ }^{70}$ M. Sarrat, D. Del Sarto, and A. Ghizzo, Europhys. Lett. 115, 45001 (2016).

${ }^{71}$ F. Califano, D. Del Sarto, and F. Pegoraro, Phys. Rev. Lett. 96, 105008 (2006)

${ }^{72}$ S. Tomita and Y. Ohira, Astrophys. J. 825, 103 (2016).

${ }^{73}$ S. Tomita, Y. Ohira, and R. Yamazaki, Astrophys. J. 886, 54 (2019).

${ }^{74}$ M. R. Feix, P. Bertrand, and A. Ghizzo, Eulerian Codes for the Vlasov Equation, Advances in Kinetic Theory and Computing, Series on Advances in Mathematics for Applied Sciences, edited by B. Perthame (World Scientific Publishing, 1994), Vol. 22.

${ }^{75}$ F. Filbet and E. Sonnendrücker, J. Comput. Phys. 150, 247 (2003).

${ }^{76} \mathrm{M}$. Shoucri and R. Gagne, J. Comput. Phys. 27, 315 (1978).

${ }^{77}$ B. Izrar, A. Ghizzo, P. Betrand, E. Fijalkow, and M. Feix, Comput. Phys. Commun. 52, 375 (1989).

${ }^{78}$ E. Fijalkow, Comput. Phys. Commun. 116, 336 (1999).

${ }^{79}$ F. Huot, A. Ghizzo, P. Bertrand, E. Sonnendrücker, and O. Coulaud, J. Comput. Phys. 185, 512 (2003).

${ }^{80}$ F. Filbet, E. Sonnendrücker, and P. Bertrand, J. Comput. Phys. 172, 166 (2001).

${ }^{81}$ M. Brunetti, V. Grandgirard, P. Bertrand, O. Sauter, J. Vaclavik, and L. Villard, Transport Theory and Statistical Phys. 34(3-5), 261-274 (2005).

${ }^{82}$ N. Crouseilles, M. Mehrenberger, and E. Sonnendrücker, J. Comput. Phys. 229, 1927 (2010).

${ }^{83}$ S. Labrunie, A. Carillo, and P. Bertrand, J. Comput. Phys. 200, 267 (2004).

${ }^{84}$ M. L. Begue, A. Ghizzo, and P. Bertrand, J. Comput. Phys. 151, 458 (1999). 